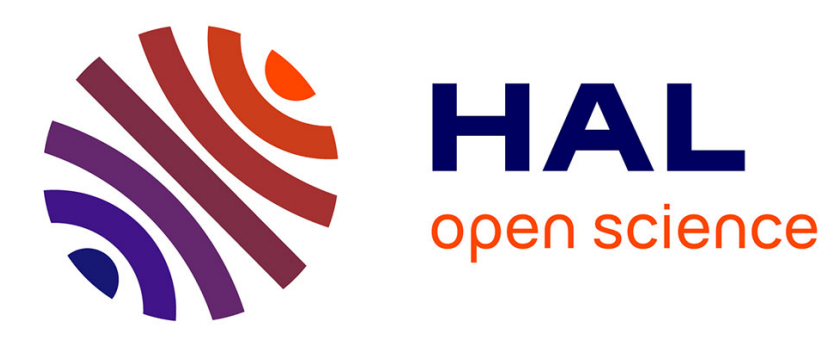

\title{
Scattering structure and Landau damping for linearized Vlasov equations with inhomogeneous Boltzmannian states
}

Bruno Despres

\section{To cite this version:}

Bruno Despres. Scattering structure and Landau damping for linearized Vlasov equations with inhomogeneous Boltzmannian states. Annales de l'Institut Henri Poincaré, 2019, pp.82 - 92. hal-01985113

\section{HAL Id: hal-01985113 \\ https: / hal.sorbonne-universite.fr/hal-01985113}

Submitted on 17 Jan 2019

HAL is a multi-disciplinary open access archive for the deposit and dissemination of scientific research documents, whether they are published or not. The documents may come from teaching and research institutions in France or abroad, or from public or private research centers.
L'archive ouverte pluridisciplinaire $\mathbf{H A L}$, est destinée au dépôt et à la diffusion de documents scientifiques de niveau recherche, publiés ou non, émanant des établissements d'enseignement et de recherche français ou étrangers, des laboratoires publics ou privés. 


\title{
Scattering structure and Landau damping for linearized Vlasov equations with inhomo- geneous Boltzmannian states
}

\author{
Bruno Després
}

Mathematics Subject Classification (2010). 35B35, 35L60, 82D10.

Keywords. Vlasov-Poisson-Ampère equation, scattering theory, LippmannSchwinger operator, Linear Landau damping, non constant Boltzmannian states.

\begin{abstract}
We study the linearized Vlasov-Poisson-Ampère equation for non constant Boltzmannian states with one region of trapped particles in dimension one and construct the eigenstructure in the context of the scattering theory. This is based on the use of semi-discrete variables (moments in velocity) and it leads to a new Lippmann-Schwinger variational equation. The continuity in quadratic norm of the operator is proved, and the well-posedness is proved for a small value of the scaling parameter. It gives a proof of Linear Landau damping for inhomogeneous Boltzmannian states. The linear HMF model is an example.
\end{abstract}

\section{Introduction}

The goal is, by means of an original scattering structure for the VlasovAmpère equation, to construct the first proof of linear Landau damping $[37,13,52]$ for a particular family of inhomogeneous Boltzmannian states $f(x, v, t)=n_{0}(x) \exp \left(-v^{2} / 2\right)+g(x, v, t)$. This work contributes to the solution of two problems addressed in [7] which are, for non homogeneous profiles, the construction are quasi-modes and the long time behavior of the Vlasov equation. Other works on the stability of the Vlasov equation for non homogeneous states are in $[50,6,39,43]$, with particular mention to $[7,3]$ on the dynamics of the HMF equation around inhomogeneous backgrounds and to the recent exposure [8]. The stability of the linear and non linear Vlasov equation is studied by numerical methods in many works, let us mention $[40,7]$ where trapped particles are discussed and $[17,23,24]$ for various tests cases. 
The scattering structure is written as linear Schrodinger equation [16, $55,29]$ and it is an extension of a previous work [18] on the linearized Vlasov-Poisson-Ampère equation with homogeneous states, but without explicit mention nor to scattering theory neither to linear Schrodinger equation. It seems that the artillery of abstract scattering theory per se $[33,38,29,55$, $25,49]$ has not been used in the literature for the study of linearized VlasovPoisson equation, at least not in $[5,19,48,21,10,11,42]$. See however the recent contribution [44] in a different direction for the Navier-Stokes equation around rapidly rotating Oseen vortices. In our case, instead of relying on general theories such as the Weil theorem $[55,33]$ for compact perturbations (see remarks at the end of Section 2.2) and the abstract Kato-Birman theory [49][page 22] for trace class operators or ways to construct the generalized wave operators [55][page 3], we focus on the explicit calculation of the eigenstructure. The keystone of this work is the derivation of an original integral equation, the Lippmann-Schwinger equation [49] of the problem. Once the Lippmann-Schwinger equation is solved, one gets a representation of the solution of the linearized Vlasov-Poisson-Ampère equation with oscillatory integrals. The method and tools are strongly related to the physical context: they are an alternative to Hamiltonian theories $[45,46,3]$ with action-angle variables popular in plasma physics, and we use a good deal of Koopmanism [35, 53, 54] to construct the eigenstructure of the different operators; the HMF model $[7,3,22]$ is one example. The extension to non linear equations is an open problem, the techniques seem so far very different $[48,47,30,32,50,39,41,43,10,9,22]$.

The principles of abstract scattering applied to the specific VlasovPoisson-Ampère considered in this work generate the Lipmann-Schwinger equation using a straightforward and mostly algebraic path. The justification of the well-posedness of the Lipmann-Schwinger equation requires much more involved analytical arguments.

Rescaled Vlasov-Ampère system. The modeling starts from the 1d-1v VlasovPoisson system

$$
\left\{\begin{array}{lll}
\partial_{t} f+v \partial_{x} f-\frac{q}{m} E \partial_{v} f=0, & t>0, & (x, v) \in \mathbb{R} \times \mathbb{R}, \\
\partial_{x} E=\varepsilon_{0}\left(\rho_{\mathrm{ref}}(x)-\int_{\mathbb{R}} f d v\right), & t>0, \quad x \in \mathbb{R} .
\end{array}\right.
$$

The unknowns are the density of electrons $f(t, x, v)$ and the electric field $E(t, x)$. One has $E=-\partial_{x} \varphi$ where $\varphi(t, x)$ is the electric potential. The constants are $q>0$ the unit positive charge, $m$ the mass of electrons and $\varepsilon_{0}$ the dielectric constant. The reference ion density is $\rho_{\text {ref }}$. After rescaling $\frac{x}{\bar{x}}=x^{\prime}$, $\frac{\underline{t}}{\bar{t}}=t^{\prime}, \frac{v}{\bar{v}}=v^{\prime}, \frac{E}{\bar{E}}=E^{\prime}, \frac{\varphi}{\varphi}=\varphi^{\prime}$ and $\frac{\rho_{\text {ref }}}{\bar{\rho}_{\text {ref }}}=\rho_{\text {ref }}^{\prime}$, one obtains

$$
\left\{\begin{array}{l}
\partial_{t^{\prime}} f+\frac{\bar{v} \bar{t}}{\bar{x}} v^{\prime} \partial_{x^{\prime}} f-\frac{q}{m} \frac{\bar{E} \bar{t}}{\bar{v}} E^{\prime} \partial_{v^{\prime}} f=0, \\
\partial_{x^{\prime}} E^{\prime}=\varepsilon_{0} \overline{x v}\left(\frac{\bar{\rho}_{\mathrm{ref}}}{\bar{v}} \rho_{\mathrm{ref}}^{\prime}(x)-\int_{\mathbb{R}} f d v^{\prime}\right), \quad E^{\prime}=-\frac{\overline{x \varphi}}{\bar{E}} \partial_{x^{\prime}} \varphi^{\prime} .
\end{array}\right.
$$

It will be mathematically convenient to consider a small parameter $\varepsilon>0$ and the specific scaling $\frac{\bar{v} \bar{t}}{\bar{x}}=1, \frac{q}{m} \frac{\bar{E} \bar{t}}{\bar{v}}=\varepsilon^{2}, \frac{\varepsilon_{0} \overline{x v}}{\bar{E}}=1, \frac{\bar{\rho}_{\text {ref }}}{\bar{v}}=1$ and $\frac{\overline{x \varphi}}{\bar{E}}=1$. 
The second relation can combined with the first and third ones under the form $q m \varepsilon_{0} \frac{\bar{x}^{2}}{\bar{v}}=\varepsilon^{2}$. Adding periodic boundary conditions, an interpretation is that we focus on solutions which are periodic over the small spatial length $\bar{x}=O(\varepsilon)$. Other combinations of the parameters are possible which reach similar rescaled equations. We rewrite the non dimensional system with original variables and consider periodic conditions at the boundaries of the space interval $I=[0,1]$

$$
\begin{cases}\partial_{t} f+v \partial_{x} f-\varepsilon^{2} E \partial_{v} f=0, & t>0, \quad(x, v) \in I \times \mathbb{R}, \\ \partial_{x} E=\rho_{\mathrm{ref}}(x)-\int_{\mathbb{R}} f d v, \quad E=-\partial_{x} \varphi, & t>0, \quad x \in I .\end{cases}
$$

One has the fundamental identity $\partial_{x}\left(\partial_{t} E-\int_{\mathbb{R}} f v d v\right)=\partial_{t}\left(\partial_{x} E\right)-\partial_{x} \int_{\mathbb{R}} f v d v=$ $-\partial_{t} \int_{\mathbb{R}} f d v-\partial_{x} \int_{\mathbb{R}} f v d v=0$. Since the third identity of the Vlasov-Poisson system above implies $\int_{I} E(t, x) d x=0$ for all $t \geq 0$, one finds that the Gauss law can be written as the Ampère law under the form $\partial_{t} E=1^{*} \int_{\mathbb{R}} v f d v$, to be compared with the one in $[34,43]$.

Notation 1.1. We will note $1^{*}: L^{2}(I) \rightarrow L_{0}^{2}(I)$ the usual projection operator such that $1^{*} g=g-\int_{I} g(x) d x$.

One gets the Vlasov-Ampère system with periodic boundary conditions

$$
\begin{cases}\partial_{t} f+v \partial_{x} f-\varepsilon^{2} E \partial_{v} f=0, & t>0, \quad(x, v) \in I \times \mathbb{R}, \\ \partial_{t} E=1^{*} \int_{\mathbb{R}} v f d v, & t>0, \quad x \in I .\end{cases}
$$

This formulation is the most convenient one for the development of the methods proposed in this work. Even if the Poisson and Ampère equations are equivalent provided the Poisson relation holds at initial time, and we will always use this assumption, the rewriting of the model problem within a scattering setting is much clearer with the Vlasov-Ampère system (1.1).

Elementary considerations and the ion positivity condition. The solutions of the Vlasov-Ampère equation are bounded $0 \leq f(t, x, v) \leq\left\|f_{\text {ini }}\right\|_{L^{\infty}(I \times \mathbb{R})}$ and satisfy the conservation of the physical energy $\frac{1}{\varepsilon^{2}} \int_{I} \int_{\mathbb{R}} f v^{2} d v d x+\int_{I} E^{2} d x$. The initial data $(f, E)$ will be considered as a perturbation of a stationary state $\left(f_{0}, E_{0}\right) v \partial_{x} f_{0}-\varepsilon^{2} E_{0}(x) \partial_{v} f_{0}=0$ with $E_{0}=-\varphi_{0}^{\prime}$. A natural possibility to represent such stationary states is

$$
f_{0}(x, v)=h\left(\frac{v^{2}}{2}-\varepsilon^{2} \varphi_{0}(x)\right)
$$

where $\varphi_{0}$ is the reference electric potential and $h$ is an arbitrary function which could be multivalued as well. We consider a single valued and regular function $h \in C^{1}(\mathbb{R})$. The Poisson equation recasts as

$$
-\varphi_{0}^{\prime \prime}(x)+\int_{\mathbb{R}} h\left(v^{2} / 2-\varepsilon^{2} \varphi_{0}(x)\right) d v=\rho_{\text {ref }}(x) .
$$

There are two ways to understand this equation. Either one assumes the ionic density $\rho_{\text {ref }}$ is given: the determination of $\varphi_{0}$ can be performed after 
reformulation as a minimization problem $\left(\widehat{h}^{\prime}=-\frac{h}{\varepsilon^{2}}\right)$

$$
\varphi_{0}=\underset{\varphi \in H_{0}^{1}(I)}{\operatorname{argmin}}\left(\int_{I}\left[\frac{1}{2} \varphi^{\prime}(x)^{2}-\rho_{\mathrm{ref}}(x) \varphi(x)+\int_{\mathbb{R}} \widehat{h}\left(v^{2} / 2-\varepsilon^{2} \varphi_{0}(x)\right) d v\right] d x\right)
$$

in the space in $H_{0}^{1}(I):=H^{1}(I)_{\text {per }} \cap\left\{\int_{I} \varphi(x) d x=0\right\}$. This problem is well posed under standard conditions [4] on the convexity of $\widehat{h}$. The second way, which is the one adopted in this work because it simplifies the mathematical analysis, is to assume that $\varphi_{0}$ is given, and in this case the ionic density is given by (1.3). However a condition is required for physical relevance, that is $\rho_{\text {ref }} \geq 0$ : indeed the periodic function $\varphi_{0}^{\prime \prime}$ necessarily takes both signs, so $\rho_{\text {ref }} \geq 0$ does not hold automatically. Nevertheless is it sufficient to require what we call hereafter the ion positivity condition

$$
\varphi_{0}^{\prime \prime}(x)<\int_{\mathbb{R}} h\left(v^{2} / 2\right) d v, \quad \forall x \in I .
$$

Indeed (1.4) guarantees that $\rho_{\text {ref }} \geq 0$ for $\varepsilon>0$ small enough.

Linearization and the rest state. We consider a linearization under the form $f(t, x, v)=f_{0}(x, v)+g(t, x, v)$ and $E(t, x)=E_{0}(x)+F(t, x)$, inject in (1.1) and drop the quadratic terms. It yields the model linearized Vlasov-PoissonAmpère system (1.5)-(2.1) studied in this work

$$
\begin{cases}\partial_{t} g+v \partial_{x} g-\varepsilon^{2} E_{0} \partial_{v} g-\varepsilon^{2} F \partial_{v} f_{0}=0, & t>0, \quad(x, v) \in I \times \mathbb{R}, \\ \partial_{t} F=1^{*} \int_{\mathbb{R}} v g d v, & t>0, \quad x \in I, \\ \partial_{x} F=-\int_{\mathbb{R}} g d v, & t>0, \quad x \in I,\end{cases}
$$

equipped with periodic boundary conditions. A first family of solutions $\left(g_{\mathrm{st}}, F_{\mathrm{st}}\right)$ to (1.5) corresponds to stationary solutions such that $\partial_{t}=0$.

Lemma 1.2. Stationary solutions of (1.5) can be parametrized under the form

$$
\left\{\begin{array}{l}
g_{\mathrm{st}}(x, v)=-\varepsilon^{2} h^{\prime}\left(v^{2} / 2-\varepsilon^{2} \varphi_{0}(x)\right) \varphi_{\mathrm{st}}(x)+h_{1}\left(v^{2} / 2-\varepsilon^{2} \varphi_{0}(x)\right), \\
F_{\mathrm{st}}^{\prime}(x)=-\int_{\mathbb{R}} g_{\mathrm{st}}(x, v) d v
\end{array}\right.
$$

where $h$ is such that (1.2) holds and $h_{1}$ is an arbitrary function. The potential $\varphi_{\text {st }}\left(\varphi_{\text {st }}^{\prime}=-F_{\text {st }}\right)$ is solution to the equation

$$
-\varphi_{\mathrm{st}}^{\prime \prime}(x)-\varepsilon^{2}\left(\int_{\mathbb{R}} h^{\prime}\left(v^{2} / 2-\varepsilon^{2} \varphi_{0}(x)\right) d v\right) \varphi_{\mathrm{st}}(x)=-\int_{\mathbb{R}} h_{1}\left(v^{2} / 2-\varepsilon^{2} \varphi_{0}(x)\right) d v .
$$

Proof. Take a pair $(g, F)$ stationary solution of $(1.5)$

$$
\begin{cases}v \partial_{x} g-\varepsilon^{2} E_{0} \partial_{v} g-\varepsilon^{2} F \partial_{v} f_{0}=0, & (x, v) \in I \times \mathbb{R}, \\ \partial_{x} F=-\int_{\mathbb{R}} g d v, & x \in I,\end{cases}
$$

and define $\widehat{g}(x, v)=g(x, v)+\varepsilon^{2} h^{\prime}\left(v^{2} / 2-\varepsilon^{2} \varphi_{0}(x)\right) \varphi(x)$ where $\varphi^{\prime}(x)=-F(x)$. In view of (1.2), one has $\left(v \partial_{x}-\varepsilon^{2} E_{0} \partial_{v}\right) \widehat{g}=\left(v \partial_{x}-\varepsilon^{2} E_{0} \partial_{v}\right) g+v \varepsilon^{2} h^{\prime}\left(v^{2} / 2-\right.$ $\left.\varepsilon^{2} \varphi_{0}(x)\right) \varphi^{\prime}(x)=\varepsilon^{2} F \partial_{v} f_{0}-v \varepsilon^{2} h^{\prime}\left(v^{2} / 2-\varepsilon^{2} \varphi_{0}(x)\right) F(x)=0$. Therefore $\widehat{g}$ is in the kernel of the transport operator, so can be represented as $\widehat{g}(x, v)=$ $h_{1}\left(v^{2} / 2-\varepsilon^{2} \varphi_{0}(x)\right)$. The closure relation (1.7) is evident. 
Since $\varphi_{\text {st }}$ is stationary and can be non zero, no damping of the electric field shows up with this first family (1.6-1.7). Additionally the density $g_{\text {st }}$ is stationary, so the total density $f=f_{0}+g$ is constant. In the context of this work, such stationary profiles are of little interest and will not been studied. To pursue the main topic of this work which is non stationary solutions, we make additional hypothesis. The first one is the Boltzmanian hypothesis because it is compatible with the common physical sense and it helps to concentrate on the main difficulties.

Hypothesis 1.3 (Boltzmanian hypothesis). $h(w)=\exp (-w)$.

With this assumption, the reference density of electrons (1.2) is given by $f_{0}(x, v)=n_{0}(x) G(v), n_{0}(x)=\exp \left(\varepsilon^{2} \varphi_{0}(x)\right)$ and $G(v)=\exp \left(-v^{2} / 2\right)$. The Poisson-Poincaré equation (1.3) becomes $-\varphi_{0}^{\prime \prime}+\alpha^{2} \exp \left(\varepsilon^{2} \varphi_{0}\right)=\rho_{\text {ref }}$ with $\alpha^{2}=\int_{\mathbb{R}} \exp \left(-\frac{v^{2}}{2}\right) d v=\sqrt{2 \pi}$. The compatibility relation (1.7) is well posed in the sense that the term in parenthesis is negative, so the equation is elliptic coercive.

We will make one more assumption illustrated in Fig. 1. As in [6], it corresponds to a potential with one region of trapped particles. More regions of trapped particles can be included, at the price of heavier notations.

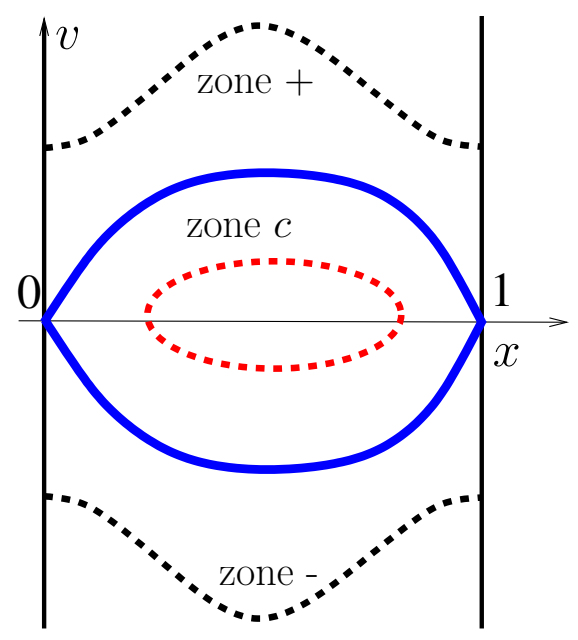

Figure 1. The separatrix in solid line $\frac{v^{2}}{2}-\varepsilon^{2} \varphi_{0}(x)=$ $-\varepsilon^{2} \varphi_{0}^{-}=-\varepsilon^{2} \varphi_{0}(0)=-\varepsilon^{2} \varphi_{0}(1)$ splits the domain in three zones. Zone + is above the separatrix. Zone - is below the separatrix. The central zone of trapped particules, indexed by the letter $c$, corresponds to closed loops that cross the horizontal axis. 
Hypothesis 1.4 (Structural assumption). The electric potential $\varphi_{0} \in W_{\text {per }}^{3, \infty}(I)$ is monotone

$$
\left\{\begin{array}{l}
\varphi_{0}^{\prime}(x)>0 \text { for } 0<x<x_{0} \\
\varphi_{0}^{\prime}(x)<0 \text { for } x_{0}<x<1
\end{array}\right.
$$

normalized by translation in the $x$ direction (i.e. $\varphi_{0}^{-}=\min _{x} \varphi_{0}=\varphi_{0}(0)$ ), strictly convex at the minimum (i.e. $\left.\varphi^{\prime \prime}(0)>0\right)$ and strictly concave at the maximum (i.e. $\varphi_{0}^{\prime \prime}\left(x_{0}\right)<0$ and $\varphi_{0}^{+}=\max _{x} \varphi_{0}=\varphi_{0}\left(x_{0}\right)$ ).

The fundamental assumption is the following.

Hypothesis 1.5 (Monotony of time of travel). The time needed for particles to travel along characteristics is a monotone function of the characteristic label. In this work we consider the sufficient condition (for justification refer to section 3.1.4)

$$
\sup _{x \neq 0, x_{0}, 1}\left(\frac{d^{2}}{d x^{2}} \sqrt{\varphi_{0}^{+}-\varphi_{0}(x)}\right)<0 .
$$

A typical example is the HMF model [7, 3, 22] for which $\varphi_{0}(x)=$ $-\cos (2 \pi x)$. A reasonable conjecture is that (1.9) is not satisfied by unstable BGK waves [43] which have an electron hole, as described in [31]. Next assumption is geometrically intuitive. Physically it rules out the stationary states (1.6).

Hypothesis 1.6. The initial data $g_{0}$ to (1.5) has zero mean value along the characteristics curves of the transport operator $v \partial_{x}-\varepsilon^{2} E_{0} \partial_{v}$ (precise statement in Proposition 4.3).

\section{Main result and ideas of the proof.}

Theorem 1.7 (Linear Landau damping around Boltzmannian state). Assume the initial data of the linearized Vlasov-Ampère system (1.5) has bounded quadratic energy (2.1) and satisfies the Gauss relation. Make assumptions 1.3, 1.4, 1.5 and 1.6. There exists a constant $\varepsilon_{*}>0$ such that for all $0<\varepsilon<\varepsilon_{*}$, then the electric field tends to zero in strong norm $\lim _{t \rightarrow \infty}\|F(t)\|_{L^{\infty}(I)}=0$. Moreover the ion density $\rho_{\text {ref }}(1.3)$ is positive.

To our knowledge, this is the first ever proof of linear Landau damping for Vlasov-Poisson-Ampere equations around a non homogeneous profile. One can strengthen the result with explicit rates of decay of the electric field, like $O\left(t^{-p}\right)$ in usual norms. It can be obtained with $p$ integration by parts in the representation formula (1.10) below, provided one justifies the regularity and summability of the integrands, see Remark 6.5.

The main idea of the proof is, with a new variable $U \approx(F, g)$, to obtain a convenient representation of the solution as

$$
U(t)=\sum_{z \in\{-,+, c\}} \sum_{k \neq 0} \int_{I_{z}^{\varepsilon}}\left(U_{0}, U_{e, k, z}^{\varepsilon}\right) e^{t \lambda_{e}, k} V_{e, 0, z}^{\varepsilon} t_{e}^{\varepsilon} d e
$$

where $U_{e, k, z}^{\varepsilon}$ denotes generalized eigenvectors of an linear hermitian operator $H_{0}^{\varepsilon}$ which represents the equations (1.5) without the coupling between the 
articles and the electric field and $V_{e, k, z}^{\varepsilon}$ denotes generalized eigenvectors of another linear hermitian operator $H^{\varepsilon}$ which represents the equations (1.5) with the coupling between the articles and the electric field. The parameters are: $z \in\{-,+, c\}$ the zone as in Figure $1 ; k \in \mathbb{Z}$ the discrete index of a Fourier mode along the characteristic curve with continuous label $e \in I_{z}^{\varepsilon}$; and $\varepsilon$ the small parameter already introduced. The exponentials $e^{t \lambda_{e, k}}$ contain pure imaginary terms since $\lambda_{e, k}=2 \mathbf{i} \pi p / t_{e}^{\varepsilon}$ where $t_{e}^{\varepsilon}>0$ is the finite travel time along the characteristic curve and $p \neq 0$ as a consequence of the Hypothesis 1.6. Phase mixing is promoted for large time $t>0$ by the oscillatory exponentials in the formula (1.10) since $t_{e}^{\varepsilon}$ is strictly monotone (increasing or decreasing) with respect to the label of the characteristics as a consequence of the Hypothesis 1.5: the result of Theorem 1.7 is a direct consequence of Formula (1.10).

The establishment of the formula (1.10) starts by understanding that the generalized eigenvectors $U_{e, k, z}^{\varepsilon}$ are easily constructed from the knowledge of the characteristic curves depicted in Figure 1: this is the easy part. The difficult part is the construction of the generalized eigenvectors $V_{e, k, z}^{\varepsilon}$ of the full set of equations. Using the philosophy of abstract scattering, they are constructed with a perturbative method which can be reduced to a Lipmann-Schwinger type equation [49]. The variational formulation ${ }^{1}$ of Lipmann-Schwinger equation is: find $a \in L_{0}^{2}(I)$ such that, for all $b \in L_{0}^{2}(I)$,

$$
(a, b)+\sum_{p \neq 0} \frac{\varepsilon^{2}}{\pi^{2} p^{2}} P . V \cdot \int_{\mathbb{R}} \frac{m_{a, b, p}^{\varepsilon}(\lambda) d \lambda}{\lambda-\sigma}+\sum_{p>0} \frac{\varepsilon^{2}}{\pi^{2} p^{2}} P . V \cdot \int_{\mathbb{R}} \frac{n_{a, b, p}^{\varepsilon}(\lambda) d \lambda}{\lambda-\sigma}=\mathcal{L}^{\varepsilon}(b),
$$

where the function $a$ is fundamentally an electric field (more precisely it is the first component of $\left.V_{e, 0, z}^{\varepsilon}\right)$ and $\sigma \in \mathbb{R}^{*}$. The terms $m_{a, b, p}^{\varepsilon}$ and $n_{a, b, p}^{\varepsilon}$ are bilinear forms with respect to $a$ and $b$ and the term $\mathcal{L}^{\varepsilon}(b)$ is a linear form with respect to $b$. The well-posedness of (1.11) is based on classical analytical considerations for the integral Hilbert transform [51], the main issue is a careful evaluation of the summability of the series (1.11). The most intricate technical issue is for the $n_{a, b, p}^{\varepsilon}$ which correspond to the trapped region of particules: a specific estimate in Hölder norm is developed for the corresponding Abel integrals [31], see Appendix B.

The organization of this work is as follows. In Section 2 we write the model problem in the context of abstract scattering theory. The spectral properties of the unperturbed operator is studied in Section 3 using a Koopmanvon-Neumann approach which makes the connection between the characteristic lines of the transport operator and the generalized eigenvectors. The property of the travel time seen as a label of the characteristics lines is studied in Section 3.1.4. The next section 4 is devoted to the study of the generalized eigenvectors of the perturbed operator by means of an original LippmannSchwinger equation. The main result is that this equation is well-posed in

\footnotetext{
${ }^{1}$ Here $(a, b)$ denotes the $L^{2}(I)$ hermitian product of two square integrable functions. The space is $L_{0}^{2}(I):=L^{2}(I) \cap\left\{\int_{I} a(x) d x=0\right\}$.
} 
$L_{0}^{2}(I)$. Section 6 is devoted to a proof of linear Landau damping for the unperturbed operator, which finishes the proof of the main Theorem 1.7. Some elementary analytical results are postponed to the appendices.

The pure imaginary number $\mathbf{i}$ satisfies $\mathbf{i}^{2}=-1$. For the sake of simplicity, some technicalities will be treated without further justification with the Dirac comb formula

$$
\sum_{k \in \mathbb{Z}} \exp (2 \mathbf{i} \pi k x)=\delta(x)
$$

or with a similar formula $\sum_{n=0}^{\infty} \psi_{n}(x) \psi_{n}(y)=\delta(x-y)$ where $\psi_{n}$ is the Hermite function [1]. The notation $\varepsilon$ is reserved for the small parameter used for rescaling. The notation $\alpha=(2 \pi)^{\frac{1}{4}}$ is reserved for the Gauss identity $\int_{\mathbb{R}} \exp \left(-v^{2} / 2\right)=\sqrt{2 \pi}=\alpha^{2}$. We shall use the generic notation $C:=C\left(\varphi_{0}\right)$ for any real constant which has a dependance solely with respect to $\varphi_{0}$. Assumptions 1.3, 1.4, 1.5 and 1.6 are made without restrictions.

\section{A scattering formulation}

We reformulate the model problem as an abstract scattering equation. This is made possible because the system (1.5) with the Boltzmanian assumption 1.3 is endowed with a weighted $L^{2}$ conservation property which can be traced back to the early works of Kruzkal-Obermann [36] and Antonov [2]

$$
\frac{d}{d t}\left(\int_{I} \int_{\mathbb{R}} \frac{g^{2}}{\varepsilon^{2} n_{0} G} d v d x+\int_{I} F^{2} d x\right)=0 .
$$

\subsection{Reformulation}

Let us define $M(x, v)=\sqrt{f_{0}(x, v)}=\sqrt{n_{0}(x) G(v)}=\exp \left(-\frac{v^{2}}{4}+\varepsilon^{2} \frac{\varphi_{0}(x)}{2}\right)$ and the function $u=\frac{g}{\varepsilon M}$. Using that $\left(v \partial_{x}-\varepsilon^{2} E_{0} \partial_{v}\right) M=0$, the model linear Vlasov-Ampère system (1.5) is rewritten as

$$
\left\{\begin{array}{lll}
\partial_{t} u+v \partial_{x} u-\varepsilon^{2} E_{0} \partial_{v} u & =-\varepsilon v M F, & t>0, \quad(x, v) \in I \times \mathbb{R}, \\
\partial_{t} F & =\varepsilon 1^{*} \int_{\mathbb{R}} u v M d v, \quad t>0, \quad x \in I,
\end{array}\right.
$$

with the energy identity $\frac{d}{d t}\left(\int_{I} \int_{\mathbb{R}} u^{2} d v d x+\int_{I} F^{2} d x\right)=0$. The Gauss law rewrites

$$
\partial_{x} F=-\varepsilon \int_{\mathbb{R}} u M d v \text {. }
$$

Define the space $L_{0}^{2}(I):=\left\{F \in L^{2}(I) \mid \int_{I} F(x) d x=0\right\}$. Let us consider that $v M \in \mathcal{L}\left(L_{0}^{2}(I), L^{2}(I \times \mathbb{R})\right)$ is a multiplication operator. Take $u \in L^{2}(I \times \mathbb{R})$ and $F \in L_{0}^{2}(I)$ : one has the identity

$$
\int_{I} \int_{\mathbb{R}} u(v M F) d v d x=\int_{I}\left(\int_{\mathbb{R}} u v M d v\right) F d x=\int_{I}\left(1^{*} \int_{\mathbb{R}} u v M d v\right) F d x .
$$

Denoting $\left(1^{*} \int_{v} v M\right) u=1^{*} \int_{\mathbb{R}} u v M d v$, it shows that $1^{*} \int_{v} v M \in \mathcal{L}\left(L^{2}(I \times \mathbb{R}), L_{0}^{2}(I)\right)$ is the adjoint operator of $v M$. We systematically introduce the pure imaginary number $\mathbf{i}^{2}=-1$ to obtain compatibility with more standard notations 
in scattering theory $[33,38,29,55,25,49]$. Define the space of complex-valued functions

$$
X=L^{2}(I \times \mathbb{R}) \times L_{0}^{2}(I) .
$$

One can recast the linear Vlasov-Ampère equations $(2.2)$ as $\partial_{t} U(t)=\mathbf{i} H^{\varepsilon} U(t)$ where the unknown is $U=\left(\begin{array}{c}u \\ F\end{array}\right) \in X$ and the anti-symmetric operator is

$$
\mathbf{i} H^{\varepsilon}=\left(\begin{array}{c|c}
-v \partial_{x}+\varepsilon^{2} E_{0} \partial_{v} & -\varepsilon v M \\
\hline \varepsilon 1^{*} \int_{v} v M & 0
\end{array}\right) .
$$

In order to keep a physical interpretation of the left hand side of the equation, the imaginary number $\mathbf{i}$ is factor of the Hamiltonian operator and not of the time derivative: it is only a matter of taste. One has the decomposition of operators $\mathbf{i} H^{\varepsilon}=\mathbf{i} H_{0}^{\varepsilon}+\varepsilon \mathbf{i} K$ where

$$
\mathbf{i} H_{0}^{\varepsilon}=\left(\begin{array}{c|c}
-v \partial_{x}+\varepsilon^{2} E_{0} \partial_{v} & 0 \\
\hline 0 & 0
\end{array}\right) \text { and } \varepsilon \mathbf{i} K=\left(\begin{array}{c|c}
0 & -\varepsilon v M \\
\hline \varepsilon 1^{*} \int_{v} v M & 0
\end{array}\right)
$$

The unperturbed operator $\mathbf{i} H_{0}^{\varepsilon}$ models the dynamics of the particles along the characteristics without the strong interaction with the electric field, but with some knowledge of the electric field through the term $\varepsilon^{2} E_{0} \partial_{v}$. The perturbation operator $\varepsilon \mathbf{i} K$ models the strong interaction between the particles and the electric field. Writing in Section 2.2 all quantities with moments against Hermite functions, we will see that the operator $K$ is in the velocity direction realized as an infinite matrix with finite rank. So $K$ can be seen as a compact operator in $v$. Actually compactness of the perturbation $K$ in the space direction $x$ can be obtained by averaging lemmas, as in [20] which extends earlier results $[27,26]$. The term $\varepsilon^{2} E_{0} \partial_{v}$ is still part of the unperturbed operator $\mathbf{i} H_{0}^{\varepsilon}$ because it is not a compact perturbation. The reason there is a $\varepsilon^{2}$ in front of $E_{0} \partial_{v}$ is because it is the only way that was found to insure the physically sound ion positivity condition (1.4). This way of writing the "free operator" $\mathbf{i} H_{0}^{\varepsilon}$, original with respect to the literature due to the $\varepsilon$ dependance, is convenient for our purposes.

\subsection{Hermite representation of the eigenstructure}

We concentrate on the explicit construction of generalized eigenvectors $U_{\lambda}^{\varepsilon}$ of the free Hamiltonian $\mathbf{i} H_{0}^{\varepsilon} U_{\lambda}^{\varepsilon}=\lambda^{\varepsilon} U_{\lambda}^{\varepsilon}$, and of generalized eigenvectors $V_{\lambda}^{\varepsilon}$ of the full Hamiltonian $\mathbf{i} H^{\varepsilon} V_{\lambda}^{\varepsilon}=\lambda^{\varepsilon} V_{\lambda}^{\varepsilon}$. We decide to construct a convenient basis of the space $L^{2}(I \times \mathbb{R})$ with moments against Hermite functions as proposed in the seminal works $[14,15]$, with recent extensions in $[28,18]$. Moreover it appears that the equation which defines the generalized eigenvectors of $\mathbf{i} H_{0}^{\varepsilon}$ can more easily be defined if one has in mind the structure of the characteristic lines of the transport operator because the generalized eigenvectors can be interpreted as some Dirac masses which move along the characteristic lines. This approach, which makes an explicit connection between a transport/Liouville equation and the underlying Hilbert structure, is 
called the $\mathrm{KvN}$ approach, giving full credit to a formalization of quantum mechanics achieved by Koopman [35] and von Neuman [53, 54]. It is sometimes referred to as Koopmanism.

The Hermite polynomials $H_{n}(v)=(-1)^{n} G(v)^{-1} \frac{d^{n}}{d v^{n}} G(v)$ are orthonormal with respect to the Maxwellian weight $G(v)$, see $[1,28]$. The degree of $H_{n}$ is $n$. The parity of $H_{n}$ is the parity of $n$. Hermite polynomials are orthogonal with respect to the Maxwellian weight $\int H_{n}(v) H_{m}(v) G(v) d v=(2 \pi)^{\frac{1}{2}} n ! \delta_{n m}$ for $n, m \in \mathbb{N}$. One has the recursion formula $H_{n+1}(v)=v H_{n}(v)-H_{n-1}(v)$. Hermite polynomials are a Hilbert basis of $\left\{f \in L_{\text {loc }}^{2}(\mathbb{R}) \mid \int_{\mathbb{R}} f^{2}(v) G(v) d v<\infty\right\}$. Define for convenience $I_{n}(v)=(2 \pi)^{-\frac{1}{4}} n !^{-\frac{1}{2}} H_{n}(v)$ and the Hermite functions $\psi_{n}(v)=I_{n}(v) G^{\frac{1}{2}}(v)$ which constitute a Hilbert basis of $L^{2}(\mathbb{R})$. The family $\left(\psi_{n}\right)_{n \in \mathbb{N}}$ is by construction orthonormal: $\int_{\mathbb{R}} \psi_{p}(v) \psi_{q}(v) d v=\delta_{p q}$. The first terms of the series are

$\psi_{0}(v)=\frac{G^{\frac{1}{2}}(v)}{\alpha}, \quad \psi_{1}(v)=\frac{v G^{\frac{1}{2}}(v)}{\alpha}, \quad \psi_{2}(v)=\frac{\left(v^{2}-1\right) G^{\frac{1}{2}}(v)}{\alpha \sqrt{2}}, \quad \alpha=(2 \pi)^{\frac{1}{4}}$.

The recursion formula becomes after rescaling

$$
v \psi_{n}(v)=\sqrt{n+1} \psi_{n+1}(v)+\sqrt{n} \psi_{n-1}(v), \quad n \in \mathbb{N} .
$$

Another fundamental relation $[1,28]$ writes

$$
\psi_{n}^{\prime}(v)=\frac{1}{2}\left(-\sqrt{n+1} \psi_{n+1}(v)+\sqrt{n} \psi_{n-1}(v)\right), \quad n \in \mathbb{N} .
$$

The system (2.2) is therefore rewritten as

$$
\left\{\begin{array}{llll}
\partial_{t} u+v \partial_{x} u-\varepsilon^{2} E_{0} \partial_{v} u & =-\alpha n_{0}(x) \psi_{1}(v) F, & t>0, \quad(x, v) \in I \times \mathbb{R}, \\
\partial_{t} F & =\alpha\left(n_{0} \psi_{1}\right)^{*} u, & & t>0, \quad x \in I
\end{array}\right.
$$

where $\left(n_{0} \psi_{1}\right)^{*}$ is the adjoint of the multiplication operator by $n_{0}(x) \psi_{1}$, in the spaces $L_{0}^{2}(I) \times L^{2}(I \times \mathbb{R})$.

Assuming in view of the energy identity that $u(t) \in L^{2}(I \times \mathbb{R})$, we define the moments $\alpha_{n}(t) \in L^{2}(I)$ by $u(t, x, v)=\sum_{n} u_{n}(t, x) \psi_{n}(v)$ and $u_{n}=$ $\int_{\mathbb{R}} u \psi_{n} d v$. By definition $\|u\|_{L^{2}(I \times \mathbb{R})}^{2}=\sum_{n \in \mathbb{N}}\left\|u_{n}\right\|_{L^{2}(I)}^{2}$. One constructs the vector $U$ where $F$ is the first component

$$
U(t, \cdot)=\left(F(t, \cdot), u_{0}(t, \cdot), u_{1}(t, \cdot), u_{2}(t, \cdot), \ldots\right)^{t} .
$$

The symmetric matrix $A^{t}=A=\left(a_{i j}\right)_{(i, j) \in \mathbb{N}^{2}}$ with $a_{i j}=\delta_{|i-j|, 1} \sqrt{\max (j-2,0)}$ corresponds to the multiplication by $v$ and the antisymmetric matrix $B^{t}=$ $-B$ with $b_{i j}=\frac{1}{2}(-1)^{j-i} a_{i j}$ corresponds to the operator $\partial_{v}$. That is

$$
A=\left(\begin{array}{lllll}
0 & 0 & 0 & 0 & \ldots \\
0 & 0 & 1 & 0 & \ldots \\
0 & 1 & 0 & 2^{\frac{1}{2}} & \ldots \\
0 & 0 & 2^{\frac{1}{2}} & 0 & \ldots \\
0 & 0 & 0 & 3^{\frac{1}{2}} & \ldots \\
\ldots & \ldots & \ldots & \ldots & \ldots
\end{array}\right) \text { and } B=\frac{1}{2}\left(\begin{array}{lllll}
0 & 0 & 0 & 0 & \ldots \\
0 & 0 & 1 & 0 & \ldots \\
0 & -1 & 0 & 2^{\frac{1}{2}} & \ldots \\
0 & 0 & -2^{\frac{1}{2}} & 0 & \ldots \\
0 & 0 & 0 & -3^{\frac{1}{2}} & \ldots \\
\ldots & \ldots & \ldots & \ldots & \ldots
\end{array}\right) \text {. }
$$


The rank-2 matrix $D$ corresponds to the coupling terms in the right hand side of $(2.8)$

$$
D=\left(\begin{array}{llllll}
0 & 0 & \alpha 1^{*} \sqrt{n_{0}(x)} & 0 & 0 & \ldots \\
0 & 0 & 0 & 0 & 0 & \ldots \\
-\alpha \sqrt{n_{0}(x)} & 0 & 0 & 0 & 0 & \ldots \\
0 & 0 & 0 & 0 & 0 & 0 \\
0 & 0 & 0 & 0 & 0 & 0 \\
\ldots & \ldots & \ldots & \ldots & \ldots & \ldots
\end{array}\right)=-D^{t} .
$$

With these notations the system (2.8) is recast as $\partial_{t} U=\mathbf{i} H^{\varepsilon} U$ where $\mathbf{i} H^{\varepsilon}=$ $\mathbf{i}\left(H_{0}^{\varepsilon}+\varepsilon K\right), \mathbf{i} H_{0}^{\varepsilon}=-A \partial_{x} U+\varepsilon^{2} E_{0}(x) B U$ and $\mathbf{i} K U=D U$.

\subsection{Spaces}

The Hilbert space (2.4) is identified with

$$
X=\left\{U=\left(\alpha_{n}\right)_{n \in \mathbb{N}} \in L^{2}(I)^{\mathbb{N}} \text { with } \alpha_{0} \in L_{0}^{2}(I), \sum_{n \in \mathbb{N}}\left\|\alpha_{n}\right\|_{L^{2}(I)}^{2}<\infty\right\} .
$$

The natural norm in $X$ is $\|U\|^{2}=\sum_{n}\left\|\alpha_{n}\right\|_{L^{2}(I)}^{2}$. The sesquilinear product is $\left(U^{1}, U^{2}\right)=\sum_{n} \int_{I} \alpha_{n}^{1}(x) \overline{\alpha_{n}^{2}(x)} d x$ for $U^{1}, U^{2} \in X$. A space larger than $X$ is $X_{\text {loc }}=L_{0}^{2}(I) \times L^{2}(I)^{\mathbb{N}}$. We shall also consider a smaller space $X_{0}$ compactly embedded in $X$, all its elements have compact support with respect to the index $n$

$$
X_{0}=\left\{U \in X \mid \alpha_{n} \in W_{\text {per }}^{1, \infty}(I) \text { for } n \leq N \text { and } \alpha_{n}=0 \text { for } N<n\right\} .
$$

One has the embeddings $X_{0} \subset X \subset X_{\text {loc }}$.

\subsection{Spectral reduction of $A$}

The operator/matrix $A$ can be viewed as an unbounded hermitian operator over the space $l^{2}$. We will use the notation that $e_{p}=(0, \ldots, 0,1,0, \ldots)^{t}$, $p=0,1, \ldots$, where the coefficient 1 is in the $p$-th position. We say that $W \in \mathbb{R}^{\mathbb{N}}$ is a generalized eigenvector of $A$ if it satisfies the eigenequation $A W=\mu W, \quad \mu \in \mathbb{R}$. If $W \in l_{2}$, then $W$ is a classical eigenvector and $\mu$ is in the discrete spectrum.

Proposition 2.1. The matrix $A$ admits one null eigenvector $e_{0}\left(A e_{0}=0\right)$ in the discrete spectrum. For all $\mu \in \mathbb{R}, U_{\mu}$ is in the continuous spectrum

$$
U_{\mu}=\left(0, \psi_{0}(\mu), \psi_{1}(\mu), \psi_{2}(\mu), \ldots\right)^{t}, \quad A U_{\mu}=\mu U_{\mu} .
$$

Proof. Evident from the recurrence relation (2.6) for Hermite functions.

Proposition 2.2. These eigenvectors are complete: for all $U \in l^{2}$, one has the spectral decomposition $U=U \cdot e_{0} e_{0}+\int_{\mathbb{R}} U \cdot U_{\mu} U_{\mu} d \mu$ and $|U|^{2}=U \cdot U=$ $\left|U \cdot e_{0}\right|^{2}+\int_{\mathbb{R}}\left|U \cdot U_{\mu}\right|^{2} d \mu$.

Proof. Hermite functions $\left(\psi_{n}(\mu)\right)_{n \in \mathbb{N}}$ are a Hilbert basis of $L^{2}(\mathbb{R})$.

Lemma 2.3 (Consequence of formula (2.7)). One has $B U_{\lambda}=-\partial_{\lambda} U_{\lambda}$. 


\section{Spectral reduction of $i H_{0}^{\varepsilon}$}

The goal is to extended the previous results to the operator $\mathbf{i} H_{0}^{\varepsilon}$ viewed as an unbounded Hermitian operator over $X$. Since we desire to be specific in this work, we consider the case of study described in Figure 1 with one region of trapped particles. The separatrix is the curve $\frac{v^{2}}{2}-\varepsilon^{2} \varphi_{0}(x)=-\varepsilon^{2} \varphi_{0}^{-}$.

\subsection{Eigenstructure}

The Koopman-von Neuman philosophy is exactly what is needed to obtain an explicit representation of the generalized eigenstructure of $\mathbf{i} H_{0}^{\varepsilon}=-A \partial_{x}+$ $\varepsilon^{2} E_{0}(x) B$ in relation with a convenient characteristic equation.

- One notices that the first line of the infinite matrices $A$ and $B$ vanishes. So any function of the form

$$
u_{0}(\cdot)=w(\cdot) e_{0} \in V, \quad w \in L_{0}^{2}(I),
$$

is an eigenvector with eigenvalue 0 , therefore in the discrete spectrum.

- To construct generalized eigenvectors of $\mathbf{i} H_{0}^{\varepsilon}$, we start from the formula

$$
U(x)=\tau(x) U_{\mu(x)}=\tau(x)\left(0, \psi_{0}(\mu(x)), \psi_{1}(\mu(x)), \psi_{2}(\mu(x)), \ldots\right)^{t}
$$

and try to find some functions $x \mapsto \tau(x)$ and $x \mapsto \mu(x)$ so that $\mathbf{i} H_{0} \varepsilon U=\lambda U$ for a given $\lambda \in \mathbf{i} \mathbb{R}$. It comes from the representation with moments of a Dirac mass $u(x, v)=\tau(x) \delta(v-\mu(x))$ at a varying vertical position (this method is interpreted as Koopmanism). The algebra detailed in the next proposition is a zoom in a small interval $\left(x_{1}, x_{2}\right) \subset I$. It will be extended to $I$ in a second stage.

Proposition 3.1. Take $0<x_{1}<x_{2}<1$. Assume that

$$
-(\tau(x) \mu(x))^{\prime}=\lambda \tau(x), \quad x_{1}<x<x_{2}
$$

with the characteristic equation

$$
\frac{1}{2} \mu(x)^{2}-\varepsilon^{2} \varphi_{0}(x)=e \in \mathbb{R}, \quad x_{1}<x<x_{2} .
$$

Then $U_{\lambda}(x):=\tau(x) U_{\mu(x)}$ satisfies $\mathbf{i} H_{0}^{\varepsilon} U_{\lambda}(x)=\lambda U_{\lambda}(x)$ for $x_{1}<x<x_{2}$.

Proof. By construction one has $A U(x)=\mu(x) U(x)$ from $(2.12)$ and $B U(x)=$ $-\partial_{\lambda} U(x)$ from Lemma 2.3. So

$$
\begin{gathered}
\mathbf{i} H_{0}^{\varepsilon} U_{\lambda}(x)=-\partial_{x}(A U(x))+\varepsilon^{2} E_{0}(x) B U(x) \\
=-\partial_{x}\left(\tau(x) \mu(x) U_{\mu(x)}\right)+\varepsilon^{2} E_{0}(x) \tau(x) B U_{\mu(x)} \\
=-(\tau(x) \mu(x))^{\prime} U_{\mu(x)}-\tau(x) \mu(x) \mu^{\prime}(x)\left(\partial_{\lambda} U_{\lambda}\right)_{\lambda=\mu(x)}+\varepsilon^{2} E_{0}(x) \tau(x) B U_{\mu(x)} \\
=-(\tau(x) \mu(x))^{\prime} U_{\mu(x)}+\tau(x)\left[\mu(x) \mu^{\prime}(x)+\varepsilon^{2} E_{0}(x)\right] B U_{\mu(x)} .
\end{gathered}
$$

Using (3.1-3.2), one gets $\mathbf{i} H_{0}^{\varepsilon} U_{\lambda}(x)=\lambda \tau(x) U_{\mu(x)}+0=\lambda U_{\lambda}(x)$ for $x_{1}<x<$ $x_{2}$. The proof is ended.

The next steps extend the construction in the entire interval $I$ with the periodic boundary condition. At inspection of the figure 1 one distinguishes three zones in the construction. 
3.1.1. Zone + . In zone + the characteristics lines are mono-valued functions of the $x$ variable. It corresponds to $e>-\varepsilon^{2} \varphi_{0}^{-}$and positive velocities. Then $\mu$ in (3.2) is function of $x$ with parameters $e$ and $\varepsilon$

$$
\mu_{e}^{\varepsilon}(x)=\sqrt{2\left(e+\varepsilon^{2} \varphi_{0}(x)\right)} \geq \sqrt{2\left(e+\varepsilon^{2} \varphi_{0}^{-}\right)}>0 .
$$

This function is positive and periodic. The index $e$, also called a label in plasma physics literature, is the value of the kinetic energy at the point $x_{*}$ such that the electric potential vanishes $\varphi_{0}\left(x_{*}\right)=0$, that is $e=\mu_{e}\left(x_{*}\right)^{2} / 2=$ $v^{2}\left(x_{*}\right)^{2} / 2$.

It is convenient to define a new variable $y(x)=\int_{0}^{x} \mu_{e}^{\varepsilon}(s)^{-1} d s$. Since $\mu_{e}^{\varepsilon}$ is the dimension of a velocity, then $y$ has the dimension of a time: it is the time spent by a particle which travels along the characteristics. The total time spent by the particle along the characteristic is $t_{e}^{\varepsilon}=\int_{0}^{1} \mu_{e}^{\varepsilon}(s)^{-1} d s>0$. In the plasma physics literature $[46,8,7] y(x) / t_{e}$ is called the angle variable [3][page 5]. Equipped with these notations, one can now find the solutions of equation (3.1-3.2) over the interval $I$.

Proposition 3.2. In zone + , the periodic solutions $(\mu, \tau)$ of (3.1-3.2) are $\mu=$ $\mu_{e}^{\varepsilon}$ given by (3.3) and $\tau=\tau_{e, k}^{\varepsilon}$

$$
\tau_{e, k}^{\varepsilon}(x):=\frac{1}{t_{e}^{\varepsilon} \mu_{e}^{\varepsilon}(x)} \exp \left(2 \mathbf{i} \pi k \frac{\int_{0}^{x} \mu_{e}^{\varepsilon}(s)^{-1} d s}{t_{e}^{\varepsilon}}\right) .
$$

Remark 3.3. If $\varphi_{0} \equiv 0$, then $\frac{\int_{0}^{x} \mu_{e}^{\varepsilon}(s)^{-1} d s}{t_{e}^{\varepsilon}}=x$. In this case $\tau_{e, k}^{\varepsilon}(x)=\exp (2 i \pi k x)$ is the usual Fourier mode and is independent of $e$ and $\varepsilon$.

Proof. Equation (3.2) is evident. Equation (3.1) recasts as $-\mu(\tau \mu)^{\prime}(x)=$ $\lambda(\tau \mu)$ that is with the time variable $-\frac{d}{d y}(\tau \mu)=\lambda(\tau \mu)$. The general solution is an exponential. Since one looks for periodic functions, one obtains $\left(\tau \mu_{e}\right)(y)=$ $\frac{1}{L} \exp (2 \mathbf{i} \pi k y / y(1))$ with $\lambda=-2 i \pi k / y(1)$ where $k \in \mathbb{Z}$ and $L$ is an arbitrary factor. Taking $L=t_{e}^{\varepsilon}$ and going back to the original variable, the proof of the claim is ended.

One obtains a first family

$$
U_{e, k,+}^{\varepsilon}(x)=\frac{1}{t_{e}^{\varepsilon} \mu_{e}^{\varepsilon}(x)} \exp \left(2 \mathbf{i} \pi k \frac{\int_{0}^{x} \mu_{e}^{\varepsilon}(s)^{-1} d s}{t_{e}^{\varepsilon}}\right) U_{\mu_{e}^{\varepsilon}(x)} .
$$

Proposition 3.4. Let $e \in\left(-\varepsilon^{2} \varphi_{0}^{-}, \infty\right)$ and $k \in \mathbb{Z}$. The vectorial function $U_{e, k,+}^{\varepsilon} \in X_{\text {loc }}$ is a generalized eigenvector of $\mathbf{i} H_{0}^{\varepsilon}$

$$
\mathbf{i} H_{0}^{\varepsilon} U_{e, k,+}^{\varepsilon}=\lambda_{e, k}^{\varepsilon} U_{e, k,+}^{\varepsilon}, \quad \lambda_{e, k}^{\varepsilon}=-\frac{2 \mathbf{i} \pi k}{t_{e}^{\varepsilon}} \in \mathbf{i} \mathbb{R} .
$$

The vectorial identity (3.5) is made of a countable number of differentiable relations. 
3.1.2. Zone -. The construction is the same, but with the negative root of the characteristic equation (3.2) and still with the restriction $e>-\varepsilon^{2} \varphi_{0}^{-}$. One obtains

$$
U_{e, k,-}^{\varepsilon}(x)=\frac{1}{t_{e}^{\varepsilon} \mu_{e}^{\varepsilon}(x)} \exp \left(-2 \mathbf{i} \pi k \frac{\int_{0}^{x} \mu_{e}^{\varepsilon}(s)^{-1} d s}{t_{e}^{\varepsilon}}\right) U_{-\mu_{e}(x)} .
$$

One obtains readily.

Proposition 3.5. Let $e \in\left(-\varepsilon^{2} \varphi_{0}^{-}, \infty\right)$ and $k \in \mathbb{Z}$. The vectorial function $U_{e, k,-}^{\varepsilon} \in X_{\text {loc }}$ is a generalized eigenvector of $\mathbf{i} H_{0}^{\varepsilon}$, that is one has $\mathbf{i} H_{0}^{\varepsilon} U_{e, k,-}^{\varepsilon}=$ $\lambda_{e, k}^{\varepsilon} U_{e, k,-}^{\varepsilon}$ with $\lambda_{e, k}^{\varepsilon}=-\frac{2 \mathbf{i} \pi k}{t_{e}^{\varepsilon}}$.

3.1.3. Zone $c$. In view of the fact that the characteristic lines are closed (see figure 1), a modification is needed to define correctly the generalized eigenvectors in the central zone for trapped particles. The idea is to combine one " +" contribution (3.4) and on "-" contribution (3.6), because the characteristics make a loop in the central zone. One restricts the range

$$
e \in\left(-\varepsilon^{2} \varphi_{0}^{-},-\varepsilon^{2} \varphi_{0}^{+}\right), \quad \varphi_{0}^{-}=\min _{x \in I} \varphi_{0}(x), \quad \varphi_{0}^{+}=\max _{x \in I} \varphi_{0}(x)
$$

and defines

$$
\mu_{e}^{\varepsilon}(x)=\sqrt{2 \max \left(e+\varepsilon^{2} \varphi_{0}(x), 0\right)} \geq 0 .
$$

One tries to construct generalized eigenvectors under the form

$$
U(x)=\tau^{+}(x) U_{\mu_{e}^{\varepsilon}(x)}-\tau^{-}(x) U_{-\mu_{e}^{\varepsilon}(x)}
$$

where $\tau^{ \pm}$are unknown functions. Notice that $\mu_{e}^{\varepsilon}$ has a compact support: $\mu_{e}^{\varepsilon}(x)=0$ for $e+\varepsilon^{2} \varphi_{0}(x) \leq 0$. Considering (3.7) and Assumption 1.8, the function $\mu_{e}^{\varepsilon}$ vanishes at two endpoints defined by $\mu_{e}^{\varepsilon}\left(a_{e}^{\varepsilon}\right)=\mu_{e}^{\varepsilon}\left(b_{e}^{\varepsilon}\right)=0$ and $<a_{e}^{\varepsilon}<b_{e}^{\varepsilon}<1$. One has $\mu_{e}^{\varepsilon}(x)>0$ for $a_{e}^{\varepsilon}<x<b_{e}^{\varepsilon}$ and $\mu_{e}^{\varepsilon}(x)=0$ for $0 \leq x \leq$ $a_{e}^{\varepsilon}$ and $b_{e} \leq x \leq 1$. By imposing the condition that $\tau^{+}(x)=\tau^{-}(x)=0$ for $0 \leq x<a_{e}^{\varepsilon}$ and $b_{e}^{\varepsilon}<x \leq 1$, it insures that $U$ (3.8) vanishes identically (in $x$ ) outside the support (in $x$ ) of the characteristic line with level (or label) $e$. This idea is the main difference with the construction in zones + and -. Let us take

$$
\tau^{+}(x)=\frac{1}{\mu_{e}^{\varepsilon}(x)} \exp \left(-\lambda \int_{a_{e}^{\varepsilon}}^{x} \mu_{e}^{\varepsilon}(s)^{-1} d s\right), \quad a_{e}^{\varepsilon}<x<b_{e}^{\varepsilon} .
$$

Proposition 3.1 yields $\mathbf{i} H_{0} \tau^{+}(x) U_{\mu_{e}(x)}=\lambda \tau^{+}(x) U_{\mu_{e}(x)}$ for $a_{e}<x<b_{e}$. Similarly take with a minus sign

$$
\tau^{-}(x)=\frac{-1}{\mu_{e}^{\varepsilon}(x)} \exp \left(\lambda \int_{a_{e}^{\varepsilon}}^{x} \mu_{e}^{\varepsilon}(s)^{-1} d s\right), \quad a_{e}^{\varepsilon}<x<b_{e}^{\varepsilon} .
$$

Proposition 3.1 yields $\mathbf{i} H_{0}^{\varepsilon} \tau^{-}(x) U_{-\mu_{e}(x)}^{\varepsilon}=\lambda \tau^{-}(x) U_{-\mu_{e}(x)}^{\varepsilon}$ for $a_{e}<x<b_{e}$. The linear combination (3.8) satisfies $\mathbf{i} H_{0}^{\varepsilon} U(x)=\lambda U^{\varepsilon}(x)$ for $x \neq a_{e}^{\varepsilon}, b_{e}^{\varepsilon}$. The 
infinite vector $U$ exhibits by construction a divergence at $x=a_{e}^{\varepsilon}$ and $x=b_{e}^{\varepsilon}$, since $\mu_{e}\left(a_{e}\right)=\mu_{e}\left(b_{e}\right)=0$. More precisely for $0<a_{e}^{\varepsilon}<x<b_{e}^{\varepsilon}<1$

$$
\mu_{e}^{\varepsilon}(x)=\varepsilon \sqrt{2\left(\varphi_{0}(x)-\varphi_{0}\left(a_{e}\right)\right.} \approx \varepsilon \sqrt{2 \varphi_{0}^{\prime}\left(a_{e}\right)} \sqrt{x-a_{e}}, \text { for } x \approx\left(a_{e}^{\varepsilon}\right)^{+}
$$

and

$$
\mu_{e}^{\varepsilon}(x)=\varepsilon \sqrt{2\left(\varphi_{0}(x)-\varphi_{0}\left(b_{e}\right)\right.} \approx \varepsilon \sqrt{-2 \varphi_{0}^{\prime}\left(b_{e}\right)} \sqrt{b_{e}-x}, \text { for } x \approx\left(b_{e}^{\varepsilon}\right)^{-} .
$$

In particular $U \notin X_{\text {loc }}$ because the inverse of the square root is not square integrable. Let $e$ be in the range (3.7) and define

$$
\lambda_{e, k}^{\varepsilon}=\frac{-2 \mathbf{i} \pi k}{t_{e}^{\varepsilon}} \text { for } k \in \mathbb{Z}, \quad t_{e}^{\varepsilon}=2 \int_{a_{e}^{\varepsilon}}^{b_{e}^{\varepsilon}} \mu_{e}^{\varepsilon}(s)^{-1} d s .
$$

The factor 2 in front of the integral is because characteristics make a loop in the central zone, see Figure 1. Define infinite vector almost everywhere in $x$ as follows. If $x \in\left(a_{e}^{\varepsilon}, b_{e}^{\varepsilon}\right)$ then

$$
\begin{gathered}
U_{e, k, c}^{\varepsilon}(x)=\frac{1}{t_{e}^{\varepsilon} \mu_{e}^{\varepsilon}(x)} \exp \left(2 \mathbf{i} \pi k \frac{\int_{a_{e}^{\varepsilon}}^{x} \mu_{e}^{\varepsilon}(s)^{-1} d s}{t_{e}^{\varepsilon}}\right) U_{\mu_{e}^{\varepsilon}(x)} \\
+\frac{1}{t_{e}^{\varepsilon} \mu_{e}^{\varepsilon}(x)} \exp \left(-2 \mathbf{i} \pi k \frac{\int_{a_{e}^{\varepsilon}}^{x} \mu_{e}^{\varepsilon}(s)^{-1} d s}{t_{e}^{\varepsilon}}\right) U_{-\mu_{e}^{\varepsilon}(x)}
\end{gathered}
$$

and $U_{e, k, c}^{\varepsilon}(x)=0$ for $x \in\left[0, a_{e}^{\varepsilon}\right) \cup\left(b_{e}^{\varepsilon}, 1\right]$.

Proposition 3.6. Let $e \in\left(-\varepsilon^{2} \varphi_{0}^{+},-\varepsilon^{2} \varphi_{0}^{-}\right)$and $k \in \mathbb{Z}$. One has the regularity $U_{e, k, c}^{\varepsilon} \in L^{p}(I)^{\mathbb{N}}$ for $1 \leq p<2$ and $A U_{e, k, c}^{\varepsilon} \in C^{0}(I)^{\mathbb{N}}$. Moreover $\left(U_{e, k, c}^{\varepsilon}, \lambda_{k, e}^{\varepsilon}\right)$ is a generalized eigenpair of the operator $\mathbf{i} H_{0}^{\varepsilon}=-A \partial_{x}+\varepsilon^{2} E_{0} B$ in a weak sense

$$
\left(A U_{e, k, c}^{\varepsilon}, W^{\prime}\right)+\varepsilon^{2}\left(E_{0} B U_{e, k, c}^{\varepsilon}, W\right)=\lambda_{e, k}^{\varepsilon}\left(U_{e, k, c}^{\varepsilon}, W\right), \quad \forall W \in X_{0} .
$$

Remark 3.7. The identity (3.12) can be decomposed in a countable number of differentiable (in a weak sense) relations between functions in $L^{p}(I)$ with $1 \leq p<2$. This is an important difference with (3.5) which is in strong form.

Proof. The proof proceeds in three steps.

- One has the decomposition $U_{e, k, c}^{\varepsilon}=\left(0, \alpha_{0}, \alpha_{1}, \ldots\right)_{n \in \mathbb{N}}$ with

$$
\begin{gathered}
\alpha_{n}(x)=\frac{1}{t_{e}^{\varepsilon} \mu_{e}^{\varepsilon}(x)} \exp \left(2 \mathbf{i} \pi k \frac{\int_{a_{e}^{\varepsilon}}^{x} \mu_{e}^{\varepsilon}(s)^{-1} d s}{t_{e}^{\varepsilon}}\right) \psi_{n}\left(\mu_{e}^{\varepsilon}(x)\right) \\
+\frac{1}{t_{e}^{\varepsilon} \mu_{e}^{\varepsilon}(x)} \exp \left(-2 \mathbf{i} \pi k \frac{\int_{a_{e}^{\varepsilon}}^{x} \mu_{e}^{\varepsilon}(s)^{-1} d s}{t_{e}^{\varepsilon}}\right) \psi_{n}\left(-\mu_{e}^{\varepsilon}(x)\right)
\end{gathered}
$$

for $a_{e}^{\varepsilon}<x<b_{e}^{\varepsilon}$ and $\alpha_{n}(x)=0$ for $x \in\left[0, a_{e}^{\varepsilon}\right) \cup\left(b_{e}^{\varepsilon}, 1\right]$. Since $\mu_{e}^{\varepsilon}$ has a square root singularity at $a_{e}^{\varepsilon}$ and $b_{e}^{\varepsilon}$, then $\alpha_{n} \in L^{p}(I)$ for all $n \in \mathbb{N}$ and $1 \leq p<2$. 
- Next, as a consequence of (2.12), one has that

$$
\begin{gathered}
A U_{e, k, c}^{\varepsilon}(x)=\frac{1}{t_{e}^{\varepsilon}} \exp \left(2 \mathbf{i} \pi k \frac{\int_{a_{e}^{\varepsilon}}^{x} \mu_{e}^{\varepsilon}(s)^{-1} d s}{t_{e}^{\varepsilon}}\right) U_{\mu_{e}^{\varepsilon}(x)} \\
-\frac{1}{t_{e}^{\varepsilon}} \exp \left(-2 \mathbf{i} \pi k \frac{\int_{a_{e}^{\varepsilon}}^{x} \mu_{e}^{\varepsilon}(s)^{-1} d s}{t_{e}^{\varepsilon}}\right) U_{-\mu_{e}^{\varepsilon}(x)}, \quad x \in\left(a_{e}^{\varepsilon}, b_{e}^{\varepsilon}\right) .
\end{gathered}
$$

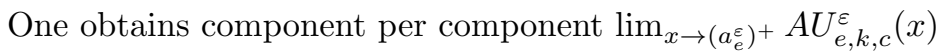

$$
\begin{aligned}
& =\frac{1}{t_{e}^{\varepsilon}}\left(U_{\mu_{e}^{\varepsilon}\left(a_{e}^{\varepsilon}\right)}-U_{-\mu_{e}^{\varepsilon}\left(a_{e}^{\varepsilon}\right)}\right)=\frac{1}{t_{e}^{\varepsilon}}\left(U_{0}^{\varepsilon}-U_{0}^{\varepsilon}\right)=0 \text {. Similarly } \\
& \lim _{x \rightarrow\left(b_{e}^{\varepsilon}\right)^{-}} A U_{e, k, c}^{\varepsilon}(x) \\
& =\frac{1}{t_{e}^{\varepsilon}}\left(\exp \left(2 \mathbf{i} \pi k \frac{\int_{a_{e}^{\varepsilon}}^{b_{e}^{\varepsilon}} \mu_{e}^{\varepsilon}(s)^{-1} d s}{t_{e}^{\varepsilon}}\right) U_{\mu_{e}^{\varepsilon}\left(b_{e}^{\varepsilon}\right)}-\exp \left(-2 \mathbf{i} \pi k \frac{\int_{a_{e}^{\varepsilon}}^{b_{e}^{\varepsilon}} \mu_{e}^{\varepsilon}(s)^{-1} d s}{t_{e}^{\varepsilon}}\right) U_{-\mu_{e}^{\varepsilon}\left(b_{e}^{\varepsilon}\right)}\right) \\
& =\frac{1}{t_{e}^{\varepsilon}}\left((-1)^{k} U_{0}-(-1)^{k} U_{0}\right)=0 .
\end{aligned}
$$

Since $U_{e, k, c}^{\varepsilon}(x)=0$ for $x<a_{e}^{\varepsilon}$ or $b_{e}^{\varepsilon}<x$, one has the continuity of $A U_{e, k, c}^{\varepsilon}$ at $a_{e}^{\varepsilon}$ and $b_{e}^{\varepsilon}$. Therefore $A U_{e, k, c}^{\varepsilon}$ is a continuous function, component wise.

- Since $A U_{e, k, c}^{\varepsilon}$ is continuous, the derivative in the sense of distribution of $A U_{e, k, c}^{\varepsilon}$ is almost everywhere equal to the point wise derivative, so (3.12) holds for all test function $W \in X_{0}$. The proof is ended.

3.1.4. Fine properties of $t_{e}^{\varepsilon}$. This section is devoted to the study of the function $e \mapsto t_{e}^{\varepsilon}$ which is the time spent by a particle which moves freely along the characteristic with label $e$. In the plasma physics literature [3][page 5], $1 / t_{e}^{\varepsilon}$ is called the frequency and enters in the definition of the action-angle variables [46]. This study is fundamental in the context of this work because the monotony of $t_{e}^{\varepsilon}$ allows to parametrize the different objects in function of the spectral variable. Once again, we make assumptions 1.3, 1.4, 1.5 and 1.6 without restriction.

There is a natural rescaling with respect to $\varepsilon$

$$
t_{e}^{\varepsilon}=\frac{1}{\varepsilon} \widehat{t}_{e / \varepsilon^{2}}, \quad \widehat{t}_{e}:=t_{e}^{1}
$$

with

$$
\begin{cases}\widehat{t}_{e}=\int_{0}^{1} \frac{d x}{\sqrt{2\left(e+\varphi_{0}(x)\right)}}, & e \in\left(-\varphi_{0}-, \infty\right) \quad \text { called the first branch, } \\ \widehat{t}_{e}=2 \int_{\widehat{a}_{e}}^{\widehat{b}_{e}} \frac{d x}{\sqrt{2\left(e+\varphi_{0}(x)\right)}}, & e \in\left(-\varphi_{0}^{+},-\varphi_{0}^{-}\right) \quad \text { called the second branch }\end{cases}
$$

where $\widehat{a}_{e}<\widehat{b}_{e}$ are the two roots of the equation $e+\varphi_{0}(x)=0$, that is $e+\varphi_{0}\left(\widehat{a}_{e}\right)=e+\varphi_{0}\left(\widehat{b}_{e}\right)=0$. One has by continuity $\widehat{a}_{-\varphi_{0}^{-}}=0, \widehat{a}_{-\varphi_{0}^{+}}=$ $b_{-\varphi_{0}^{+}}=x_{0}$ and $b_{-\varphi_{0}^{-}}=1$.

The map $e \mapsto \widehat{t}_{e}$ (and the inverse maps) are depicted in Figure 2. The monotony of the time of travel is by definition for the first branch. The major non trivial property which establishes the monotony in the second 
branch (region of trapped particles) is proved in the sequel. The reason why the time of travel is infinite for $e=-\varphi_{0}^{-}$on both branches is because the particles spend an infinite time in the region where $v \approx 0$ at the limit: refer also to Figure 1.
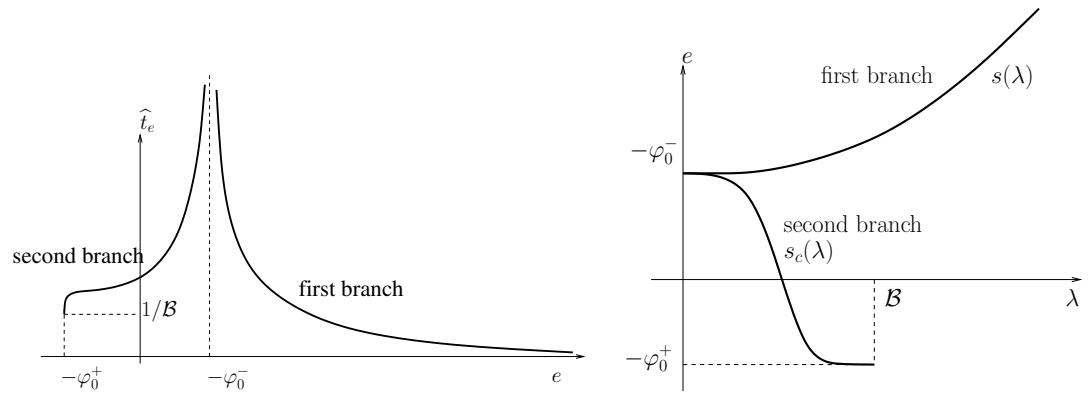

Figure 2. On the left: the total time of travel along characteristics versus the label. On the right: the label versus the frequency (i.e. the inverse of the total time of travel along characteristics).

Proposition 3.8 (Parametrization of the first branch, proof is in appendix A). The branch $e \in\left(-\varphi_{0}^{-}, \infty\right)$ can be parametrized as $e=s(\lambda)$ for $\lambda=\frac{1}{\hat{t}_{e}}$ where the function $s:(0, \infty) \rightarrow\left(-\varphi_{0}^{-}, \infty\right)$ has the properties

- The function is strictly increasing with the regularity $s \in C^{1}[0, \infty)$.

- For large $\lambda>>1$, one has $s(\lambda)=O\left(\lambda^{2}\right), s^{\prime}(\lambda)=O(\lambda), s^{\prime \prime}(\lambda)=O(1)$ and $s^{\prime \prime \prime}(\lambda)=O(1 / \lambda)$.

- For small $0<\lambda$, one has $s(\lambda)=-\varphi_{0}^{-}+\alpha_{\varphi_{0}} \exp \left(-\frac{\sqrt{\varphi_{0}^{\prime \prime}(0)}}{2 \lambda}\right)(1+\sigma(\lambda))$ where $\sigma \in C^{0}$ with $\sigma(0)=0$ and $\alpha_{\varphi_{0}}>0$ is a constant. The derivative is $s^{(n)}(\lambda)=O\left(\frac{\exp \left(-\frac{\sqrt{\varphi_{0}^{\prime \prime}(0)}}{2 \lambda}\right)}{\lambda^{2 n}}\right)$ for $n \geq 1$.

Proposition 3.9. Under assumption 1.4, the function $e \mapsto \widehat{t}_{e}$ is strictly increasing in $\left(-\varphi_{0}^{+},-\varphi_{0}^{-}\right)$.

Proof. One has the decomposition $\sqrt{2 \widehat{t}_{e}}=\int_{\widehat{a}_{e}}^{x_{0}} \frac{d x}{\sqrt{e+\varphi_{0}(x)}}+\int_{x_{0}}^{\widehat{b}_{e}} \frac{d x}{\sqrt{e+\varphi_{0}(x)}}$. We study the second term $\int_{x_{0}}^{\widehat{b}_{e}} \frac{d x}{\sqrt{e+\varphi_{0}(x)}}$ and show it is monotone. Set for convenience $\bar{e}=e+\varphi_{0}^{+} \in\left(0, \varphi_{0}^{+}-\varphi_{0}^{-}\right)$and $\psi(z)=\varphi_{0}^{+}-\varphi_{0}(x)=\varphi_{0}\left(x_{0}\right)-\varphi_{0}(x) \geq 0$. With these notations one has $\int_{x_{0}}^{\widehat{b}_{e}} \frac{d x}{\sqrt{\bar{e}+\varphi_{0}(x)}}=H(\bar{e}):=\int_{x_{0}}^{\widehat{b}_{e}} \frac{d x}{\sqrt{\bar{e}-\psi(x)}}$. Set the change of variable $\psi(x)=\bar{e} u^{2}$ for $x_{0} \leq x \leq \widehat{b}_{e}$ and $0 \leq u \leq 1$. Note that 
$\psi>0, \psi^{\prime}>0$ for $x_{0}<x<\widehat{b}_{e}$ and $\psi^{\prime}(x) d x=2 \bar{e} u d u$. So

$$
H(\bar{e})=\int_{0}^{1} \frac{2 u}{\sqrt{1-u^{2}}} g_{u}(\bar{e}) d u
$$

with

$$
g_{u}(\bar{e})=\frac{\sqrt{\bar{e}}}{\psi^{\prime}\left(\psi^{-1}\left(\bar{e} u^{2}\right)\right.} .
$$

The derivative of $g_{u}$ is $\left(z=\psi^{-1}\left(\bar{e} u^{2}\right)\right)$

$$
\frac{d}{d \bar{e}} g_{u}(\bar{e})=\frac{1}{2 \sqrt{\bar{e}} \psi^{\prime}(z)}-\sqrt{\bar{e}} \frac{\psi^{\prime \prime}(z) \psi^{\prime}(z)^{-1}\left(-u^{2}\right)}{\psi^{\prime}(z)^{2}}=\frac{1}{2 \sqrt{\bar{e}} \psi^{\prime}(z)}\left(1-2 \frac{\psi^{\prime \prime}(z) \psi(z)}{\psi^{\prime}(z)^{2}}\right) \text {. }
$$

One has $(\sqrt{\psi})^{\prime}=\frac{\psi^{\prime}}{2 \sqrt{\psi}}$ and $2(\sqrt{\psi})^{\prime \prime}=\frac{\psi^{\prime \prime}}{\sqrt{\psi}}-\frac{1}{2} \frac{\left(\psi^{\prime}\right)^{2}}{(\psi)^{\frac{3}{2}}}=-\frac{1}{2} \frac{\left(\psi^{\prime}\right)^{2}}{(\psi)^{\frac{3}{2}}}\left(1-2 \frac{\psi^{\prime \prime} \psi}{\left(\psi^{\prime}\right)^{2}}\right)$. It yields the formula

$$
\frac{d}{d \bar{e}} g_{u}(\bar{e})=-2 \frac{(\psi)^{\frac{3}{2}}}{\sqrt{\bar{e}}\left(\psi^{\prime}\right)^{3}}(\sqrt{\bar{\psi}})^{\prime \prime}=-2 \bar{e} \frac{u^{3}}{\left(\psi^{\prime}\right)^{3}}(\sqrt{\psi})^{\prime \prime} .
$$

Therefore the strict concavity of $\sqrt{\psi}$ yields an strictly increasing $e \mapsto g_{u}(e)$, so

$$
\frac{d}{d \bar{e}} H(\bar{e})=\int_{0}^{1} \frac{2 u}{\sqrt{1-u^{2}}} \frac{d}{d \bar{e}} g_{u}(\bar{e}) d u>0 .
$$

The same result for the second integral $\int_{\widehat{a}_{e}}^{x_{0}} \frac{d x}{\sqrt{e+\varphi_{0}(x)}}$. The proof is ended.

The monotony of $e \mapsto \widehat{t}_{e}$ on the second branch $e \in\left(-\varphi_{0}^{+},-\varphi_{0}^{-}\right)$implies that there is a limit at both ends of the interval. The limit is infinite at $-\varphi_{0}^{-}$, and it is proved in Lemma A.5 that the limit at the lower bound is finite $\widehat{t}_{-\varphi_{0}^{+}}=\frac{1}{\mathcal{B}}$. More precisely it is proved that the inverse of the lower bound of the time of travel in the region of trapped particles is

$$
\mathcal{B}=\frac{\sqrt{\psi_{0}^{\prime \prime}\left(x_{0}\right)}}{\sqrt{2} \pi}
$$

Proposition 3.10 (Parametrization of the second branch, proof in Appendix A). The branch $e \in\left(-\varphi_{0}^{+},-\varphi_{0}^{-}\right)$can be parametrized as $e=s_{c}(\lambda)$ for $\lambda=\frac{1}{t_{e}}$ where the function $s_{c}:(0, \mathcal{B}) \rightarrow\left(-\varphi_{0}^{+},-\varphi_{0}^{-}\right)$has the following properties

- The function is strictly decreasing with the regularity $s_{c} \in C^{1}[0, \mathcal{B}]$.

- For $\lambda<\mathcal{B}$, one has $s_{c}(\lambda)=-\varphi_{0}^{+}+\left(\frac{\pi \sqrt{\psi^{\prime \prime}\left(x_{0}\right)}}{2 \sqrt{2}(\sqrt{\psi})^{\prime \prime}\left(x_{0}\right)}\right)^{2}(\lambda-\mathcal{B})^{2}+O(\lambda-\mathcal{B})^{3}$ and $s_{c}^{\prime}(\lambda)=O(\lambda-\mathcal{B})$.

- for small $\lambda>0$, one has $s_{c}(\lambda)=-\varphi_{0}^{-}+\alpha_{\varphi_{0}} \exp \left(-\frac{\sqrt{\varphi_{0}^{\prime \prime}(0)}}{4 \lambda}\right)(1+\sigma(\lambda))$ where $\sigma$ is a continuous function with $\sigma(0)=0$. The derivative at order $n \geq 1$ is $s_{c}^{(n)}(\lambda)=O\left(\frac{\exp \left(-\frac{\sqrt{\varphi_{0}^{\prime \prime}(0)}}{2 \lambda}\right)}{\lambda^{2 n}}\right)$. 
3.1.5. Rescaled inverse fonctions. In view of (3.13) the rescaled inverse functions are $s^{\varepsilon}$ and $s_{c}^{\varepsilon}$

$$
s^{\varepsilon}(\lambda)=\varepsilon^{2} s(\lambda / \varepsilon) \text { and } s_{c}^{\varepsilon}(\lambda)=\varepsilon^{2} s_{c}(\lambda / \varepsilon) .
$$

The rescaled inverse functions are defined over $I_{+}^{\varepsilon}=I_{-}^{\varepsilon}=\left(-\varepsilon^{2} \varphi_{0}^{-}, \infty\right)$ and $I_{c}^{\varepsilon}=\left(-\varepsilon^{2} \varphi_{0}^{+},-\varepsilon^{2} \varphi_{0}^{-}\right)$. Some inequalities uniform with respect to $\varepsilon$ can be proved.

Lemma 3.11. One has the uniform lower bound

$$
c \lambda^{2} \leq s^{\varepsilon}(\lambda) \quad 0 \leq \lambda \text { and } 0<\varepsilon \leq 1
$$

and the uniform upper bounds

$$
\left|\left(s^{\varepsilon}\right)^{\prime}(\lambda)\right| \leq C \lambda \quad 0 \leq \lambda
$$

and

$$
\left|\left(s_{c}^{\varepsilon}\right)^{\prime}(\lambda)\right| \leq C \lambda \quad 0 \leq \lambda \leq \varepsilon \mathcal{B}
$$

Proof. By Proposition 3.8, there exists $c>0$ such that $\frac{s(\lambda)}{\lambda^{2}} \geq c$ for all $\lambda$. It yields (3.20). By Propositions 3.8 and 3.10, there exists $C>0$ such that $\left|s^{\prime}(\lambda)\right| \leq C \lambda$ and $\left|s_{c}^{\prime}(\lambda)\right| \leq C \lambda$. It yields (3.21-3.22). The proof is ended.

\subsection{Completness}

Theorem 3.12. Let $U \in X$. The spectral decomposition holds

$$
U=U \cdot e_{0} e_{0}+\sum_{z} \sum_{k \in \mathbb{Z}} \int_{e \in I_{z}^{\varepsilon}}\left(U, U_{e, k, z}^{\varepsilon}\right) U_{e, k, z}^{\varepsilon} t_{e}^{\varepsilon} d e
$$

with the Plancherel identity $\|U\|^{2}=\left(U, e_{0}\right)^{2}+\sum_{z} \sum_{k \in \mathbb{Z}} \int_{e \in I_{z}^{\varepsilon}}\left|\left(U, U_{e, k, z}^{\varepsilon}\right)\right|^{2} t_{e}^{\varepsilon} d e$.

Proof. The proof is performed for $U \in X_{0}$ by a direct computation. By density of $X_{0}$ in $X$, it will prove the result in $X$. Notice that $U \in X_{0}$ has only a finite number of non zero components which are all in $H^{1}(I)$ : since $U_{e, k}^{\varepsilon} \in L^{p}(I)^{\mathbb{N}}$ for $1 \leq p<2$, it is enough to get the integrability and summability needed to give sense to $\left(U, U_{e, k}^{\varepsilon}\right)$. The formulas $(3.23)$ and the Plancherel identity are equivalent, that is why we will prove only the first one. A last remark is that (3.23) can be checked for different lines independently one to the other: indeed $U, U_{e, k,+}^{\varepsilon}, \ldots$ are infinite vectors. The method of the proof is by successive changes of variables and the completeness of the Hermite functions.

For $U \in X_{0}$, we begin with the analysis of the integral in the zone $" z=+"$

$$
\begin{gathered}
K_{1}=\sum_{k \in \mathbb{Z}} \int_{I_{+}^{\varepsilon}}\left(U, U_{e, k,+}^{\varepsilon}\right) U_{e, k,+}^{\varepsilon}(x) t_{e}^{\varepsilon} d e \\
=\int_{I_{+}^{\varepsilon}}\left(\sum_{k \in \mathbb{Z}} \int_{0}^{1} U(y) \cdot U_{\mu_{e}^{\varepsilon}(y)} e^{2 \mathbf{i} \pi k \frac{\int_{0}^{x} \frac{d s}{\mu_{e}^{\varepsilon}(s)}-\int_{0}^{y} \frac{d s}{\mu_{e}^{\varepsilon}(s)}}{t_{e}^{\varepsilon}}} \frac{d y}{t_{e}^{\varepsilon} \mu_{e}^{\varepsilon}(y)}\right) U_{\mu_{e}^{\varepsilon}(x)} \frac{d e}{\mu_{e}^{\varepsilon}(x)} .
\end{gathered}
$$


The notation ". " is understood as the scalar or hermitian product of a complex valued vector $U(y)$ which has only a finite number of non zero components since $U \in X_{0}$ and the infinite real valued vector $U_{\mu_{e}^{\varepsilon}(y)}$. We make a change of variable $\widehat{y}_{e}^{\varepsilon}=\frac{1}{t_{e}^{\varepsilon}} \int_{0}^{y} \frac{d s}{\mu_{e}^{\varepsilon}(s)}$ such that $d \widehat{y}_{e}^{\varepsilon}=\frac{d y}{t_{e}^{\varepsilon} \mu_{e}^{\varepsilon}(y)}$. We also set $\widehat{x}_{e}^{\varepsilon}=\frac{1}{t_{e}^{\varepsilon}} \int_{0}^{x} \frac{d s}{\mu_{e}^{\varepsilon}(s)}$. Using the Dirac comb formula (1.12), one has that

$$
\begin{aligned}
& \sum_{k \in \mathbb{Z}} \int_{0}^{1} U(y) \cdot U_{\mu_{e}^{\varepsilon}(y)} \exp \left(2 \mathbf{i} \pi k \frac{\int_{0}^{x} \frac{d s}{\mu_{e}^{\varepsilon}(s)}-\int_{0}^{y} \frac{d s}{\mu_{e}^{\varepsilon}(s)}}{t_{e}^{\varepsilon}}\right) \frac{d y}{t_{e}^{\varepsilon} \mu_{e}^{\varepsilon}(y)} \\
= & \sum_{k \in \mathbb{Z}} \int_{0}^{1} U(y) \cdot U_{\mu_{e}^{\varepsilon}(y)} \exp \left(2 \mathbf{i} \pi k\left(\widehat{x}_{e}^{\varepsilon}-\widehat{y}_{e}^{\varepsilon}\right)\right) d \widehat{y}_{e}^{\varepsilon}=U(x) \cdot U_{\mu_{e}^{\varepsilon}(x)}
\end{aligned}
$$

One obtains $K_{1}=\int_{I_{+}^{\varepsilon}} U(x) \cdot U_{\mu_{e}^{\varepsilon}(x)} \frac{1}{\mu_{e}^{\varepsilon}(x)} U_{\mu_{e}^{\varepsilon}(x)} d e$. The definition of $\mu_{e}^{\varepsilon}$, that is $\frac{1}{2} \mu_{e}^{\varepsilon}(x)^{2}-\varepsilon^{2} \varphi_{0}(x)=e$, yields $d \mu_{e}^{\varepsilon}(x)=\frac{d e}{\mu_{e}^{\varepsilon}(x)}$ for every $x$. We write $\lambda=\mu_{e}^{\varepsilon}(x)$ with the bounds $\lambda \in\left(\varepsilon \sqrt{2\left(\varphi_{0}(x)-\varphi_{0}^{-}\right)}, \infty\right)$ in the first branch. Therefore $K_{1}=\int_{\varepsilon \sqrt{2\left(\varphi_{0}(x)-\varphi_{0}^{-}\right)}}^{\infty} U(x) \cdot U_{\lambda} U_{\lambda} d \lambda$ where $U_{\lambda}$ has been defined in (2.12). Similarly the integral in the zone " $z=-$ ", one has

$K_{2}=\sum_{k \in \mathbb{Z}} \int_{I_{-}^{\varepsilon}}\left(U, U_{e, k,-}^{\varepsilon}\right) U_{e, k,-}^{\varepsilon}(x) \frac{d e}{\mu_{e}^{\varepsilon}(x)}=\int_{I_{-}^{\varepsilon}} U(x) \cdot U_{-\lambda} U_{-\lambda} d \lambda$ rewritten as $K_{2}=\int_{-\infty}^{-\varepsilon \sqrt{2\left(\varphi_{0}(x)-\varphi_{0}^{-}\right)}} U(x) \cdot U_{\lambda} U_{\lambda} d \lambda$. With a little more technicalities detailed in the next proposition, the integral in the zone " $z=c "$ is

$$
K_{3}=\int_{-\varepsilon \sqrt{2\left(\varphi_{0}(x)-\varphi_{0}^{-}\right)}}^{\varepsilon \sqrt{2\left(\varphi_{0}(x)-\varphi_{0}^{-}\right)}} U(x) \cdot U_{\lambda} U_{\lambda} d \lambda .
$$

Using the orthonormality properties of the Hermite functions, one gets $K_{1}+$ $K_{2}+K_{3}=\int_{-\infty}^{\infty} U(x) \cdot U_{\lambda} U_{\lambda} d \lambda=U(x)-U(x) \cdot e_{0} e_{0}$ from which the claim is proved.

Proposition 3.13. The formula (3.24) holds.

Proof. We note $0<a_{e}^{\varepsilon}<b_{e}^{\varepsilon}<1$ the boundaries of the interval (3.9-3.10). Since $U_{\mu_{e}^{\varepsilon}}$ is now defined in (3.11) as the difference of two terms, full expansion yields four contributions. So

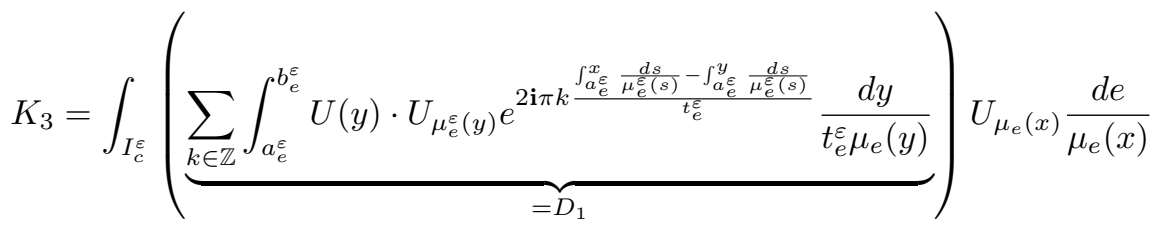

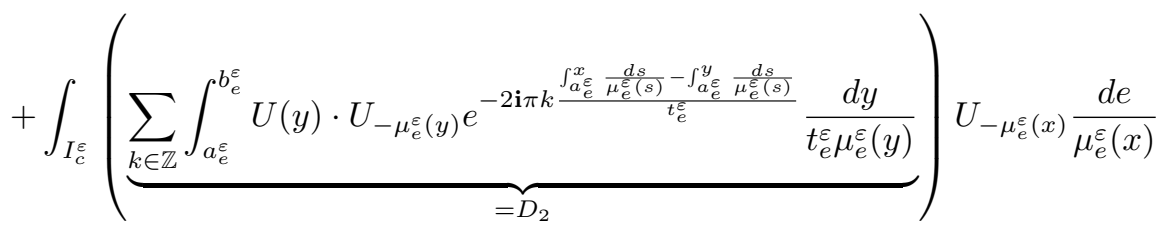




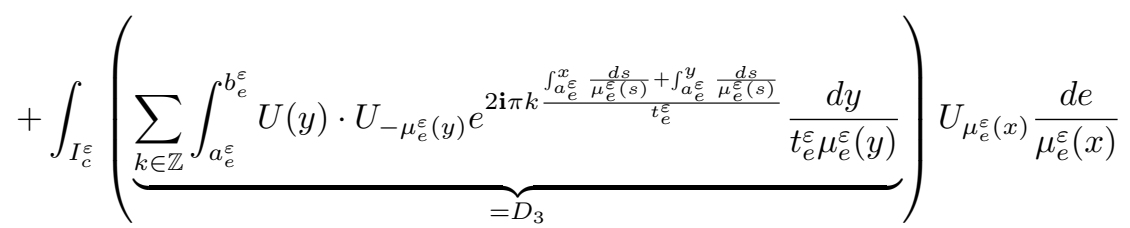

$$
\begin{aligned}
& +\int_{I_{c}^{\varepsilon}}(\underbrace{\sum_{k \in \mathbb{Z}} \int_{a_{e}^{\varepsilon}}^{b_{e}^{\varepsilon}} U(y) \cdot U_{\mu_{e}^{\varepsilon}(y)} e^{-2 \mathbf{i} \pi k \frac{\int_{a_{e}^{\varepsilon}}^{x} \frac{d s}{\mu_{e}^{\varepsilon}(s)}+\int_{a_{e}^{\varepsilon}}^{y} \frac{d s}{\mu_{e}(s)}}{t_{e}^{\varepsilon}}} \frac{d y}{t_{e}^{\varepsilon} \mu_{e}^{\varepsilon}(y)}}_{=D_{4}}) U_{-\mu_{e}^{\varepsilon}(x) \frac{d e}{\mu_{e}^{\varepsilon}(x)}} .
\end{aligned}
$$

We consider the change of variable $\widehat{y}_{e}^{\varepsilon}=\frac{2}{t_{e}^{\varepsilon}} \int_{a_{e}^{\varepsilon}}^{y} \frac{d s}{\mu_{e}^{\varepsilon}(s)}$, with $d \widehat{y}_{e}^{\varepsilon}=\frac{2 d y}{t_{e}^{\varepsilon} \mu_{e}^{\varepsilon}(y)}$. Notice that $\widehat{y}_{e}^{\varepsilon} \in[0,1]$. The same notation is used for $\widehat{x}_{e}^{\varepsilon} \in[0,1]$.

- It yields for the first term $D_{1}=\frac{1}{2} \sum_{k \in \mathbb{Z}} \int_{0}^{1} U(y) \cdot U_{\mu_{e}^{\varepsilon}(y)} \exp \left(\mathbf{i} \pi k\left(\widehat{x}_{e}^{\varepsilon}-\widehat{y}_{e}^{\varepsilon}\right)\right) d \widehat{y}_{e}^{\varepsilon}$. Splitting between even and odd $k$, one has

$$
\begin{gathered}
D_{1}=\frac{1}{2} \sum_{k \in \mathbb{Z}} \int_{0}^{1} U(y) \cdot U_{\mu_{e}^{\varepsilon}(y)} \exp \left(2 \mathbf{i} \pi k\left(\widehat{x}_{e}^{\varepsilon}-\widehat{y}_{e}^{\varepsilon}\right)\right) d \widehat{y}_{e}^{\varepsilon} \\
+\frac{1}{2} \exp \left(-\mathbf{i} \pi \widehat{x}_{e}^{\varepsilon}\right) \sum_{k \in \mathbb{Z}} \int_{0}^{1} U(y) \cdot U_{\mu_{e}^{\varepsilon}(y)} \exp \left(\mathbf{i} \pi \widehat{y}_{e}^{\varepsilon}\right) \exp \left(2 \mathbf{i} \pi k\left(\widehat{x}_{e}^{\varepsilon}-\widehat{y}_{e}^{\varepsilon}\right)\right) d \widehat{y}_{e}^{\varepsilon} .
\end{gathered}
$$

Use the Dirac comb: $D_{1}=\frac{1}{2} U(x) \cdot U_{\mu_{e}^{\varepsilon}(x)}+\frac{1}{2} \exp (-\mathbf{i} \pi \widehat{x})\left(U(x) \cdot U_{\mu_{e}^{\varepsilon}(x)} \exp (\mathbf{i} \pi \widehat{x})\right)=$ $U(x) \cdot U_{\mu_{e}^{\varepsilon}(x)}$.

- One has for similar reasons $D_{2}=U(x) \cdot U_{-\mu_{e}^{\varepsilon}(x)}$.

- The same change of variable in the third term yields

$$
\begin{gathered}
D_{3}=\frac{1}{2} \sum_{k \in \mathbb{Z}} \int_{0}^{1} U(y) \cdot U_{\mu_{e}^{\varepsilon}(y)} \exp \left(\mathbf{i} \pi k\left(\widehat{x}_{e}^{\varepsilon}+\widehat{y}_{e}^{\varepsilon}\right)\right) d \widehat{y}_{e}^{\varepsilon} \\
=\frac{1}{2} \sum_{k \in \mathbb{Z}} \int_{0}^{1} U(y) \cdot U_{\mu_{e}^{\varepsilon}(y)} \exp \left(2 \mathbf{i} \pi k\left(-\left(1-\widehat{x}_{e}^{\varepsilon}\right)+\widehat{y}_{e}^{\varepsilon}\right)\right) d \widehat{y}_{e}^{\varepsilon} \\
+\frac{1}{2} \exp \left(\mathbf{i} \pi \widehat{x}_{e}^{\varepsilon}\right) \sum_{k \in \mathbb{Z}} \int_{0}^{1} U(y) \cdot U_{\mu_{e}^{\varepsilon}(y)} \exp \left(\mathbf{i} \pi \widehat{y}_{e}^{\varepsilon}\right) \exp \left(2 \mathbf{i} \pi k\left(-\left(1-\widehat{x}_{e}^{\varepsilon}\right)+\widehat{y}_{e}^{\varepsilon}\right)\right) d \widehat{y}_{e}^{\varepsilon} .
\end{gathered}
$$

The change of variable is such that there exists $\bar{x} \in\left(a_{e}, b_{e}\right)$ (a priori $\bar{x} \neq$ $x)$ such that $\widehat{x}_{e}^{\varepsilon}=\frac{2}{t_{e}^{\varepsilon}} \int_{b_{e}^{\varepsilon}+a_{e}^{\varepsilon}-\bar{x}}^{b^{\varepsilon}} \frac{d s}{\mu_{e}^{\varepsilon}(s)}$. So $1-\widehat{x}_{e}^{\varepsilon}=1-\frac{2}{t_{e}^{\varepsilon}} \int_{b_{e}^{\varepsilon}+a_{e}^{\varepsilon}-\bar{x}}^{b^{\varepsilon}} \frac{d s}{\mu_{e}^{\varepsilon}(s)}=$ $\frac{2}{t_{e}^{\varepsilon}} \int_{a_{e}^{\varepsilon}}^{b_{e}^{\varepsilon}+a_{e}^{\varepsilon}-\bar{x}} \frac{d s}{\mu_{e}^{\varepsilon}(s)}=\frac{2}{t_{e}^{\varepsilon}} \int_{a_{e}^{\varepsilon}}^{b_{e}^{\varepsilon}} \frac{d s}{\mu_{e}^{\varepsilon}(s)}-\frac{2}{t_{e}^{\varepsilon}} \int_{b_{e}^{\varepsilon}+a_{e}^{\varepsilon}-\bar{x}}^{b^{\varepsilon}} \frac{d s}{\mu_{e}^{\varepsilon}(s)}=\frac{2}{t_{e}^{\varepsilon}} \int_{a_{e}^{\varepsilon}}^{b_{e}^{\varepsilon}+a_{e}^{\varepsilon}-\bar{x}} \frac{d s}{\mu_{e}^{\varepsilon}(s)}$. So $D_{3}=\frac{1}{2} U\left(b_{e}^{\varepsilon}+a_{e}^{\varepsilon}-\bar{x}\right) \cdot U_{\mu_{e}^{\varepsilon}\left(b_{e}^{\varepsilon}+a_{e}^{\varepsilon}-\bar{x}\right)}$

$+\frac{1}{2} \exp (\mathbf{i} \pi \widehat{x}) U\left(b_{e}^{\varepsilon}+a_{e}^{\varepsilon}-\bar{x}\right) \cdot U_{\mu_{e}^{\varepsilon}\left(b_{e}^{\varepsilon}+a_{e}^{\varepsilon}-\bar{x}\right)} \exp (\mathbf{i} \pi(1-\widehat{x}))=0$. Similarly $D_{4}=$ 0 .

- Final proof of (3.24). One can write

$$
K_{3}=\int_{I_{c}^{\varepsilon}} U(x) \cdot U_{\mu_{e}^{\varepsilon}(x)} U_{\mu_{e}^{\varepsilon}(x)} \frac{d e}{\mu_{e}^{\varepsilon}(x)}+\int_{I_{c}^{\varepsilon}} U(x) \cdot U_{-\mu_{e}^{\varepsilon}(x)} U_{-\mu_{e}^{\varepsilon}(x)} \frac{d e}{\mu_{e}^{\varepsilon}(x)}
$$




$$
\begin{gathered}
\int_{0}^{\varepsilon \sqrt{2\left(\varphi_{0}(x)-\varphi_{0}^{-}\right)}} U(x) \cdot U_{\lambda} U_{\lambda} d \lambda+\int_{-\varepsilon \sqrt{2\left(\varphi_{0}(x)-\varphi_{0}^{-}\right)}}^{0} U(x) \cdot U_{\lambda} U_{\lambda} d \lambda \\
=\int_{-\varepsilon \sqrt{2\left(\varphi_{0}(x)-\varphi_{0}^{-}\right)}}^{\varepsilon \sqrt{2\left(\varphi_{0}(x)-\varphi_{0}^{-}\right)}} U(x) \cdot U_{\lambda} U_{\lambda} d \lambda .
\end{gathered}
$$

The results proceeds by summation of $D_{1}, D_{2}, D_{3}$ and $D_{4}$. So the proof.

\section{Spectral reduction of $i H^{\varepsilon}$}

The next step is the construction of the generalized eigenvectors of $\mathbf{i} H^{\varepsilon}$. These new eigenvectors will be denoted as $V_{e, k, z}^{\varepsilon}$ with the same indices as for the $U_{e, k, z}^{\varepsilon}$, and they will be constructed by perturbation of the $U_{e, k, z}^{\varepsilon}$. This approach follows a classical approach in abstract scattering [49] and without surprise a Lippmann-Schwinger type equation shows up. A dedicated study will show the Lippmann-Schwinger equation is well posed in $L_{0}^{2}(I)$ for small $\varepsilon>0$. We will make use of elementary identities which are somehow pivotal in this section.

Lemma 4.1 (Case $k=0$ ). One has the identities where the right sides are constant with respect to $x$

$$
\exp \left(\varepsilon^{2} \varphi_{0} / 2\right) U_{s, 0, \pm}^{\varepsilon} \cdot e_{2}= \pm \frac{\exp (-s / 2)}{\alpha t_{s}^{\varepsilon}}, \quad \text { and } U_{s, 0, c}^{\varepsilon} \cdot e_{2}=0
$$

Proof. Consequence of the definitions of the eigenvectors $U_{e, k, z}$ for $k=0 \mathrm{~s}$ (3.4), (3.6) and (3.11), and of the definition of the second component of these eigenvectors which uses $\psi_{2}(v)(2.5)$. It yields $\exp \left(\varepsilon^{2} \varphi_{0}(x) / 2\right) U_{s, 0,+}^{\varepsilon}(x) \cdot e_{2}$ $=\exp \left(\varepsilon^{2} \varphi_{0}(x) / 2\right) \frac{1}{t_{s}^{\varepsilon} \mu_{s}^{\varepsilon}(x)} \frac{\mu_{s}^{\varepsilon}(x)}{\alpha} \exp \left(-\mu_{s}^{\varepsilon}(x)^{2} / 4\right)=\frac{\exp (-s / 2)}{\alpha t_{s}^{\varepsilon}} \operatorname{using}$ (3.2) for the last simplification. The two other relations are obtained similarly. The proof is ended.

In a first stage we will construct explicitly the eigenvectors and generalized eigenvectors in the null space. In a second stage we will consider generalized eigenvectors associated with non zero eigenvalues. This construction evidences the role of a specific Lippmann-Schwinger type equation. Once the Lippmann-Schwinger equation is solved, the construction becomes essentially explicit.

\subsection{The null space}

Vectors in the null space satisfy $\mathbf{i} H^{\varepsilon} V=0$. We distinguish two cases.

4.1.1. Vectors with finite norm. Let us construct a null finite eigenvector $V(x)=f(x) e_{0}+\alpha \varepsilon g(x) e_{1}$. One has

$$
\begin{gathered}
0=\mathbf{i} H^{\varepsilon} V=\left(-A \partial_{x}+\varepsilon^{2} E_{0}(x) B+\varepsilon D\right)\left(f(x) e_{0}+\alpha \varepsilon g(x) e_{1}\right) \\
=\varepsilon D f(x) e_{0}+\varepsilon\left(-A \partial_{x}+\varepsilon^{2} E_{0}(x) B\right) \alpha g(x) e_{1} .
\end{gathered}
$$


In view of the definition of the matrices $D, A$ and $B$, one gets the identity

$$
\begin{aligned}
0 & =-\alpha \sqrt{n_{0}(x)} f(x) e_{2}-\alpha g^{\prime}(x) e_{2}-\frac{1}{2} \varepsilon^{2} E_{0}(x) \alpha g(x) e_{2} \\
& =\alpha\left(-\sqrt{n_{0}(x)} f(x)-g^{\prime}(x)-\frac{1}{2} \varepsilon^{2} E_{0}(x) g(x)\right) e_{2}
\end{aligned}
$$

rewritten as $\exp \left(\varepsilon^{2} \varphi_{0}(x) / 2\right) f(x)=-g^{\prime}(x)+\frac{1}{2} \varepsilon^{2} \varphi_{0}^{\prime}(x) g(x)$. Since $f, g$ and $\varphi_{0}$ are 1-periodic functions, one has $f=-\left(\exp \left(-\varepsilon^{2} \varphi_{0} / 2\right) g\right)^{\prime}$. Notice that $f$ has necessarily a zero mean value. For $f \in L_{0}^{2}(I), g \in H_{\mathrm{per}}^{1}(I)$ is defined by integration through the above condition. We set

$$
V_{f}^{\varepsilon}(x)=f(x) e_{0}+\alpha \varepsilon g(x) e_{1}, \quad x \in I .
$$

The previous construction can be summarized as follows.

Lemma 4.2. Assume $f \in L_{0}^{2}(I)$. Then $V_{f}^{\varepsilon} \in X$ and $\mathbf{i} H^{\varepsilon} V_{f}=0$.

Proposition 4.3. Assume $U \in X$ is orthogonal to null eigenvectors $V_{f}^{\varepsilon} \in X$ for all $f \in L_{0}^{2}(I)$. Then $U$ satisfies the Gauss law (2.3).

Proof. Using the decomposition (2.9), one has that for all $f \in L_{0}^{2}(I)$

$$
\begin{aligned}
& 0=\left(U, V_{f}^{\varepsilon}\right)=\int_{I}\left(-\left(\exp \left(-\varepsilon^{2} \varphi_{0}(x) / 2\right) g(x)\right)^{\prime} F(x)+\alpha \varepsilon g(x) u_{0}(x)\right) d x \\
& =\int_{I}\left(\exp \left(-\varepsilon^{2} \varphi_{0}(x) / 2\right) F^{\prime}(x)+\alpha \varepsilon \int_{\mathbb{R}} \frac{1}{\alpha} \exp \left(-\frac{v^{2}}{4}\right) u(x, v) d v\right) g(x) d x
\end{aligned}
$$

It shows that $F^{\prime}(x)=-\varepsilon \exp \left(\varepsilon^{2} \varphi_{0}(x) / 2\right) \int_{\mathbb{R}} \exp \left(-\frac{v^{2}}{4}\right) u(x, v) d v$, which is the Gauss law (2.3). The proof is ended.

4.1.2. Generalized vectors. From (4.1) one gets

$$
\mathbf{i} K U_{e, 0, z}^{\varepsilon}=1^{*}\left(\varepsilon^{2} \exp \left(\varphi_{0} / 2\right) U_{e, 0, z}^{\varepsilon} \cdot e_{2}\right) e_{0}=0 .
$$

Since all infinite vectors $U_{e, 0, z}^{\varepsilon}$ are also in the null space of $\mathbf{i} H_{0}^{\varepsilon}$, then it is natural to set

$$
V_{e, 0, z}^{\varepsilon}=U_{e, 0, z}^{\varepsilon} \text { with the property } \mathbf{i} H^{\varepsilon} V_{e, 0, z}^{\varepsilon}=0 .
$$

Proposition 4.4. Assume $g \in L^{2}(I \times \mathbb{R})$ satisfies assumption 1.6, that is it has zero mean value along the characteristic curves of the transport operator $v \partial_{x}-\varepsilon^{2} E_{0}(x) \partial_{v}$. Then the corresponding $U \in X$ is such that $\left(U, U_{e, 0, z}^{\varepsilon}\right)=0$ for all $z$ and almost all $e$.

Proof. The proof is performed in strong form. One has

$$
\begin{gathered}
\left(U, U_{e, 0, z}^{\varepsilon}\right)=\int_{x \in I_{z}^{\varepsilon}} \sum_{n \in \mathbb{N}}\left(\int_{\mathbb{R}} g(x, v) \psi_{n}(v) d v\right) \frac{1}{t_{e}^{\varepsilon} \mu_{e}^{\varepsilon}(x)} \psi_{n}\left(\mu_{e}^{\varepsilon}(x)\right) d x \\
=\frac{1}{t_{e}^{\varepsilon}} \int_{x \in I_{z}^{\varepsilon}} \int_{\mathbb{R}} g(x, v)\left(\sum_{n \in \mathbb{N}} \psi_{n}(v) \psi_{n}\left(\mu_{e}^{\varepsilon}(x)\right)\right) d v \frac{d x}{\mu_{e}^{\varepsilon}(x)} .
\end{gathered}
$$


The representation of the Dirac mass with Hermite functions yields

$\left(U, U_{e, 0, z}^{\varepsilon}\right)=\frac{1}{t_{e}^{\varepsilon}} \int_{x \in I_{z}^{\varepsilon}} \int_{\mathbb{R}} g(x, v) \delta\left(v-\mu_{e}^{\varepsilon}(x)\right) d v \frac{d x}{\mu_{e}^{\varepsilon}(x)}=\frac{1}{t_{e}^{\varepsilon}} \int_{x \in I_{z}^{\varepsilon}} g\left(x, \mu_{e}^{\varepsilon}(x)\right) \frac{d x}{\mu_{e}^{\varepsilon}(x)}$.

Along the characteristic curve $C_{e}^{\varepsilon}$ with label $e$, the velocity is $\mu_{e}^{\varepsilon}(x)$. So one can make the change of variables $v=\mu_{e}^{\varepsilon}(x)$ and $d t=\frac{d x}{\mu_{e}^{\varepsilon}(x)}=\frac{d x}{v}$. Therefore one has the formula $\left(U, U_{e, 0, z}^{\varepsilon}\right)=\frac{1}{t_{e}^{\varepsilon}} \int_{(x, v) \in C_{e}^{\varepsilon}} g(x, v) d t$ where the integral is proportional to the mean value of $g$ along the characteristic curve. So the claim is just a convenient reformulation of Assumption 1.6 and the proof is ended.

\subsection{Non zero spectrum and Lippmann-Schwinger equation}

We study a way to construct solutions of the generalized eigenequation $\mathbf{i} H^{\varepsilon} V=$ $\lambda V$ for a non zero generalized eigenvalue $\lambda \in \mathbf{i} \mathbb{R}^{*}$, by means of a perturbation technique starting from $U_{e, k, z}^{\varepsilon}$. Let us remind the exact representation formula (3.23) for $U \in X U=U \cdot e_{0} e_{0}+\sum_{z^{\prime}} \sum_{p \in \mathbb{Z}} \int_{s \in I_{z^{\prime}}^{\varepsilon}}\left(U, U_{s, p, z^{\prime}}^{\varepsilon}\right) U_{s, p, z^{\prime}}^{\varepsilon} t_{s}^{\varepsilon} d s$ where $z^{\prime}=+,-, c$ is the index of the zone. The main idea is to start from a perturbation of $U_{e, k, z}^{\varepsilon}$ under the form

$$
V_{e, k, z}^{\varepsilon}(x)=U_{e, k, z}^{\varepsilon}(x)+a_{e, k, z}^{\varepsilon}(x) e_{0}+\sum_{z^{\prime}} \sum_{p \in \mathbb{Z}} \int_{s \in I_{z^{\prime}}^{\varepsilon}} b_{\substack{s, p, z^{\prime} \\ e, k, z}}^{\varepsilon} U_{s, p, z^{\prime}}^{\varepsilon}(x) t_{s}^{\varepsilon} d s .
$$

where the function $x \mapsto a_{e, k, z}^{\varepsilon}(x)$ and the functions $s \mapsto \underset{\substack{s, p, z^{\prime} \\ e, k, z}}{\varepsilon}$ are the unknowns. The method consists to plug formally the representation (4.4) in the eigenequation and to determine what constraints $a_{e, k, z}^{\varepsilon}$ and $\underset{\substack{s, p, z^{\prime} \\ e, k, z}}{\varepsilon}$ must satisfy. It is possible to decompose these vectorial identities into many scalar identities, all of them making sense under usual integrability conditions. The whole construction will be justified in section 5 where the existence of $a_{e, k, z}^{\varepsilon} \in L^{2}(I)$ is established after studying summability of the series.

One gets by formal calculations

$$
\begin{gathered}
\mathbf{i} H^{\varepsilon} V_{e, k, z}^{\varepsilon}=\mathbf{i} H_{0}^{\varepsilon} V_{e, k, z}^{\varepsilon}+\mathbf{i} \varepsilon K V_{e, k, z}^{\varepsilon}= \\
=\lambda_{e, k}^{\varepsilon} U_{e, k, z}^{\varepsilon}+\sum_{z^{\prime}} \sum_{p \in \mathbb{Z}} \int_{s \in I_{z^{\prime}}^{\varepsilon}} b_{s, p, z^{\prime}}^{\varepsilon} \lambda_{s, p}^{\varepsilon} U_{s, p, z}^{\varepsilon} t_{s}^{\varepsilon} d s \\
+\mathbf{i} \varepsilon K a_{e, k, z}^{\varepsilon} e_{0}+\mathbf{i} \varepsilon K U_{e, k, z}^{\varepsilon}+\mathbf{i} \varepsilon \sum_{z^{\prime}} \sum_{p \in \mathbb{Z}} \int_{\substack{s \in I_{z^{\prime}}^{\varepsilon} \\
b_{s, p, z^{\prime}}^{\varepsilon} \\
e, k, z}} K U_{s, p, z} t_{s}^{\varepsilon} d s .
\end{gathered}
$$

By definition (2.10) $\mathbf{i} K e_{p}=\exp \left(\varepsilon^{2} \varepsilon^{2} \varphi_{0} / 2\right) D e_{p}=\exp \left(\varepsilon^{2} \varphi_{0} / 2\right) \alpha\left(\delta_{p, 2} e_{0}-\right.$ $\left.\delta_{p, 0} e_{2}\right)$. Using in the previous expression, it yields

$$
\begin{aligned}
& \mathbf{i} H^{\varepsilon} V_{e, k, z}^{\varepsilon}=\lambda_{e, k}^{\varepsilon} U_{e, k, z}^{\varepsilon}+\sum_{z^{\prime}} \sum_{p \in \mathbb{Z}} \int_{s \in I_{z^{\prime}}^{\varepsilon}} b_{\substack{s, p, z^{\prime} \\
e, k, z}}^{\varepsilon} \lambda_{s, p}^{\varepsilon} U_{s, p, z}^{\varepsilon} t_{s}^{\varepsilon} d s-\varepsilon \alpha \exp \left(\varepsilon^{2} \varphi_{0} / 2\right) a_{e, k, z}^{\varepsilon} e_{2} \\
& +\varepsilon \alpha 1^{*}\left(\exp \left(\varepsilon^{2} \varphi_{0} / 2\right) U_{e, k, z}^{\varepsilon} \cdot e_{2}+\exp \left(\varepsilon^{2} \varphi_{0} / 2\right) \sum_{z^{\prime}} \sum_{p \in \mathbb{Z}} \int_{\substack{s \in I_{z^{\prime}}^{\varepsilon} \\
b_{s, k, z^{\prime}}}} U_{s, p}^{z} \cdot e_{2} t_{s}^{\varepsilon} d s\right) e_{0} .
\end{aligned}
$$


On the other hand one has

$$
\lambda_{e, k}^{\varepsilon} V_{e, k, z}^{\varepsilon}=\lambda_{e, k}^{\varepsilon} U_{e, k, z}^{\varepsilon}+\lambda_{e, k}^{\varepsilon} a_{e, k, z}^{\varepsilon} e_{0}+\sum_{z^{\prime}} \sum_{p \in \mathbb{Z}} \int_{s \in I_{z^{\prime}}^{\varepsilon}} b_{\substack{s, p, z^{\prime} \\ e, k, z}}^{\varepsilon} \lambda_{e, k} U_{s, p, z^{\prime}}^{\varepsilon} t_{s}^{\varepsilon} d s
$$

So the eigenequation $\mathbf{i} H^{\varepsilon} V_{e, k, z}^{\varepsilon}=\lambda_{e, k}^{\varepsilon} V_{e, k, z}^{\varepsilon}$ recasts as

$$
\begin{gathered}
\sum_{z^{\prime}} \sum_{p \in \mathbb{Z}} \int_{s \in I_{z^{\prime}}^{\varepsilon}} b_{s, p, z^{\prime}}^{\varepsilon}\left(\lambda_{s, k}^{\varepsilon}-\lambda_{e, k}^{\varepsilon}\right) U_{s, p, z^{\prime}}^{\varepsilon} t_{s}^{\varepsilon} d s-\alpha \varepsilon \exp \left(\varepsilon^{2} \varphi_{0} / 2\right) a_{e, k, z}^{\varepsilon} e_{2}= \\
+\alpha\left(\frac{1}{\alpha} \lambda_{e, k}^{\varepsilon} a_{e, k, z}^{\varepsilon}(x)-\varepsilon 1^{*}\left(\exp \left(\varepsilon^{2} \varphi_{0} / 2\right) U_{e, k, z}^{\varepsilon} \cdot e_{2}\right)\right. \\
\left.-\varepsilon \sum_{z^{\prime}} \sum_{p \in \mathbb{Z}} \int_{s \in I_{z^{\prime}}^{\varepsilon}} b_{s, p, z^{\prime}, z}^{\varepsilon} 1^{*}\left(\exp \left(\varepsilon^{2} \varphi_{0} / 2\right) U_{s, p, z^{\prime}}^{\varepsilon} \cdot e_{2}\right) t_{s}^{\varepsilon} d s\right) e_{0} .
\end{gathered}
$$

The right hand side is a finite linear combination of $e_{0}$ and $e_{2}$, so is a priori a function in $X$. Therefore the above equality is identified as an equality between functions in $X$ for which the spectral representation (3.23) applies. The equality of the coefficients writes

$$
\left\{\begin{array}{l}
b_{s, p, z^{\prime}}^{\varepsilon}\left(\lambda_{s, p}^{\varepsilon}-\lambda_{e, k}^{\varepsilon}\right)=\varepsilon\left(\alpha \exp \left(\varepsilon^{2} \varphi_{0} / 2\right) a_{e, k, z}^{\varepsilon} e_{2}, U_{s, p, z^{\prime}}^{\varepsilon}\right) \\
\frac{1}{\alpha} \lambda_{e, k}^{\varepsilon} a_{e, k, z}^{\varepsilon}-\varepsilon \sum_{z^{\prime}} \sum_{p \neq 0} \int_{s \in I_{z^{\prime}}^{\varepsilon}} b_{s, p, z^{\prime}}^{\varepsilon} 1^{*}\left(\exp \left(\varepsilon^{2} \varphi_{0} / 2\right) U_{s, p, z^{\prime}}^{\varepsilon} \cdot e_{2}\right) t_{s}^{\varepsilon} d s \\
=\varepsilon 1^{*}\left(\exp \left(\varepsilon^{2} \varphi_{0} / 2\right) U_{e, k, z}^{\varepsilon} \cdot e_{2}\right) .
\end{array}\right.
$$

The double sum in the second equation is for $p \neq 0$ since all terms for $p=0$ vanish in view of the identity (4.1). The solvability of this system is an issue. For a given $\varepsilon>0$, the unknowns are the functions $x \mapsto a_{e, k, z}^{\varepsilon}$ and $s \mapsto b_{\substack{s, p, z^{\prime} \\ e, k, z}}^{\varepsilon}$ for all admissible values of the parameters $(e, k, z)$. To continue the discussion one eliminates $b_{\substack{s, p, z^{\prime} \\ e, k, z}}^{\varepsilon}=\varepsilon \frac{\left(\alpha a_{e, k, z}^{\varepsilon} e_{2}, \exp \left(\varepsilon^{2} \varphi_{0} / 2\right) U_{s, p, z^{\prime}}^{\varepsilon}\right)}{\lambda_{s, p}^{\varepsilon}-\lambda_{e, k}^{\varepsilon}}$. It is mandatory that $\lambda_{s, p}^{\varepsilon}-\lambda_{e, k}^{\varepsilon} \neq 0$ for this formula to make sense. Let us plug in the second equation of (4.5). It yields the formal integral relation

$$
\begin{aligned}
& \frac{1}{\alpha} \lambda_{e, k}^{\varepsilon} a_{e, k, z}^{\varepsilon}-\varepsilon^{2} \sum_{z^{\prime}} \sum_{p \neq 0} \int_{s \in I_{z^{\prime}}^{\varepsilon}} \frac{\left(\alpha a_{e, k, z}^{\varepsilon} e_{2}, \exp \left(\varepsilon^{2} \varphi_{0} / 2\right) U_{s, p, z^{\prime}}^{\varepsilon}\right)}{\lambda_{s, p}^{\varepsilon}-\lambda_{e, k}^{\varepsilon}} \\
& \times 1^{*}\left(\exp \left(\varepsilon^{2} \varphi_{0} / 2\right) U_{s, p, z^{\prime}}^{\varepsilon} \cdot e_{2}\right) t_{s}^{\varepsilon} d s=\varepsilon 1^{*}\left(\exp \left(\varepsilon^{2} \varphi_{0} / 2\right) U_{e, k, z}^{\varepsilon} \cdot e_{2}\right) .
\end{aligned}
$$

This integral equation is only formal at this stage for many reasons. A first reason is one must provide a treatment of vanishing denominators $\lambda_{s, p}^{\varepsilon}-$ $\lambda_{e, k}^{\varepsilon}=0$. The second reason is that a sum with respect to $p$ shows up so one needs to prove the summability. This will justified a posteriori by the completeness of the eigenvectors.

To answer the first question the integrals with respect to $s$ will be defined as principal values [51] or Hilbert transforms. The notation P.V. will denote a principal value. Since it has been proved in Section 3.1.4 that $\lambda_{s, p}$ is a 
monotone function of $s$, this makes sense.

The summability will be proved in a second stage: it is conditionally convergent to a specific reordering of the various terms, cf. Remark 5.8.

In summary the construction of $V_{e, k, z}^{\varepsilon}$ amounts to solving the series of problems:

For $k \neq 0, z \in\{+,-, c\}$ and $e \in I_{z}^{\varepsilon}$, solve the integral equation for $a=a_{e, k, z}^{\varepsilon} \in L_{0}^{2}(I)$

$$
\begin{aligned}
\frac{1}{\alpha} \lambda_{e, k}^{\varepsilon} a & -\varepsilon^{2} \sum_{z^{\prime}} \sum_{p \neq 0} P . V . \int \frac{\left(\alpha a e_{2}, \exp \left(\varepsilon^{2} \varphi_{0} / 2\right) U_{s, p, z^{\prime}}^{\varepsilon}\right)}{\lambda_{s, p}^{\varepsilon}-\lambda_{e, k}^{\varepsilon}} \\
& \times 1^{*}\left(\exp \left(\varepsilon^{2} \varphi_{0} / 2\right) U_{s, p, z^{\prime}}^{\varepsilon} \cdot e_{2}\right) t_{s}^{\varepsilon} d s \\
& =\varepsilon 1^{*}\left(\exp \left(\varepsilon^{2} \varphi_{0} / 2\right) U_{e, k, z}^{\varepsilon} \cdot e_{2}\right) .
\end{aligned}
$$

By comparison with the general theory of scattering [49] [page 98], we refer to this integral equation as the Lippmann-Schwinger equation of the problem.

\section{The Lippmann-Schwinger equation}

The well posedness for small $\varepsilon$ of the Lippmann-Schwinger equation is established for the equivalent weak form, using a combination of elementary analytical techniques which are somehow independent of the method that was used to derive the equation. The key is to obtain "good" $L^{\infty}$ estimates in Section 5.1. The completeness of the whole family of generalized eigenvectors will be proved with "good" $L^{2}$ estimates in Section 5.3. So far, no clear physical interpretation has been found for these estimates which are based on hard analysis.

\subsection{Variational formulation}

Some symmetry hidden in the strong form of the Lipmann-Schwinger equation is revealed by the variational (or weak) formulation. To obtain more compact notations where the kernels in (4.6) are decomposed between a "space" part which depends on $x$ and a "integral" part which does not depend on $x$, we define

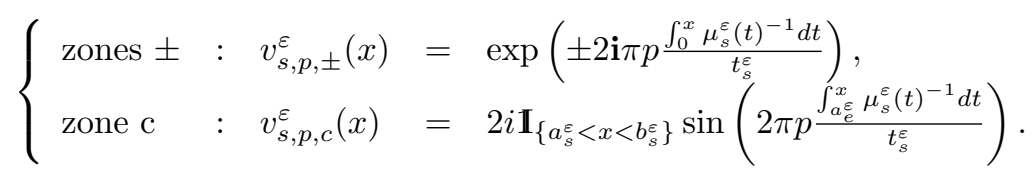

If $\varphi_{0}=0$ or $\varepsilon=0$, then $v_{s, p,+}^{\varepsilon}(x)=v_{s,-p,-}^{\varepsilon}(x)=\exp (2 \mathbf{i} \pi p x)$ are both equal to the same Fourier mode. In the general case which is our concern from now on, one gets using the identity $\frac{1}{2} \mu_{s}^{2}(x)-\varepsilon^{2} \varphi_{0}(x)=s$

$$
e^{\varepsilon^{2} \varphi_{0}(x) / 2} U_{s, p, z}^{\varepsilon}(x) \cdot e_{2}=v_{s, p, z}^{\varepsilon}(x) \frac{\exp (-s / 2)}{t_{s}^{\varepsilon} \alpha} .
$$


The variational form of the different terms that arise in (4.6) writes after integration against $b \in L_{0}^{2}(I)$ as

$$
\begin{aligned}
& F_{a, b, p}^{\varepsilon}(\mu)=P . V . \int_{s \in I_{+}^{\varepsilon}} \frac{\left(a, v_{s, p,+}^{\varepsilon}\right)\left(v_{s, p,+}^{\varepsilon}, b\right)}{\lambda_{s, p}^{\varepsilon}-\mu} \frac{\exp (-s)}{t_{s}^{\varepsilon}} d s \\
& \quad+P . V . \int_{s \in I_{-}^{\varepsilon}} \frac{\left(a, v_{s,-p,-}^{\varepsilon}\right)\left(v_{s,-p,-}^{\varepsilon}, b\right)}{\lambda_{s,-p}^{\varepsilon}-\mu} \frac{\exp (-s)}{t_{s}^{\varepsilon}} d s
\end{aligned}
$$

and similarly

$$
\begin{aligned}
& G_{a, b, p}^{\varepsilon}(\mu)=P . V . \int_{s \in I_{c}^{\varepsilon}} \frac{\left(a, v_{s, p, c}^{\varepsilon}\right)\left(v_{s, p, c}^{\varepsilon}, b\right)}{\lambda_{s, p}^{\varepsilon}-\mu} \frac{\exp (-s)}{t_{s}^{\varepsilon}} d s \\
& \quad+P . V . \int_{s \in I_{c}^{\varepsilon}} \frac{\left(a, v_{s,-p, c}^{\varepsilon}\right)\left(v_{s,-p, c}^{\varepsilon}, b\right)}{\lambda_{s,-p}^{\varepsilon}-\mu} \frac{\exp (-s)}{t_{s}^{\varepsilon}} d s .
\end{aligned}
$$

$F_{a, b, p}^{\varepsilon}(\mu)$ and $G_{a, b, p}^{\varepsilon}(\mu)$ are bilinear forms with respect to $a, b \in L_{0}^{2}(I)$. We define also a linear form

$$
l^{\varepsilon}(b)=\frac{\exp (-e / 2)}{t_{e}^{\varepsilon}}\left(v_{e, k, z}^{\varepsilon}, b\right) \text { for } b \in L_{0}^{2}(I) .
$$

A variational form of the Lippmann-Schwinger (4.6) is as follows:

Take $k \neq 0, z \in\{+,-, c\}$ and $e \in I_{z}^{\varepsilon}$; for $\mu=\lambda_{e, k}^{\varepsilon} \neq 0$, find $a \in L_{0}^{2}(I)$ solution of the variational Lippmann-Schwinger formulation

$$
\mu(a, b)-\varepsilon^{2} \sum_{p \neq 0} F_{a, b, p}^{\varepsilon}(\mu)-\varepsilon^{2} \sum_{p>0} G_{a, b, p}^{\varepsilon}(\mu)=\varepsilon l^{\varepsilon}(b), \quad \forall b \in L_{0}^{2}(I) .
$$

It is clear that the issue is to obtain good bounds on the series of $F_{a, b, p}^{\varepsilon}(\mu)$ and on the series of $G_{a, b, p}^{\varepsilon}(\mu)$, since then, the coefficient $\varepsilon^{2}$ will be enough to get the coercivity of the bilinear form.

5.1.1. Properties of $F_{a, b, p}^{\varepsilon}(\mu)$. The $F_{a, b, p}^{\varepsilon}(\mu)$ correspond to the two regions of non trapped particles, and it appears that the estimates below are natural.

Lemma 5.1. Let $\mu \in \mathbf{i} \mathbb{R}^{*}$ and $p \neq 0$. One has $F_{a, b, p}^{\varepsilon}(\mu)=\frac{\mu}{(2 \pi p)^{2}} f_{a, b, p}^{\varepsilon}\left(\frac{\mu}{2 \pi p}\right)$ where

$$
\begin{gathered}
f_{a, b, p}^{\varepsilon}(\mu)=-P . V . \int_{s \in I_{+}^{\varepsilon}} \frac{\left(a, v_{s, p,+}^{\varepsilon}\right)\left(v_{s, p,+}^{\varepsilon}, b\right)}{1 / t_{s}^{\varepsilon}-i \mu} \exp (-s) d s \\
\quad+\text { P.V. } \int_{s \in I_{-}^{\varepsilon}} \frac{\left(a, v_{s,-p,-}^{\varepsilon}\right)\left(v_{s,-p,-}^{\varepsilon}, b\right)}{-1 / t_{s}^{\varepsilon}-i \mu} \exp (-s) d s .
\end{gathered}
$$

Proof. With the notation $c_{s, p}^{\varepsilon}=\left(a, v_{s, p,+}^{\varepsilon}\right)\left(v_{s, p,+}^{\varepsilon}, b\right)=\left(a, v_{s,-p,-}^{\varepsilon}\right)\left(v_{s,-p,-}^{\varepsilon}, b\right)$ which takes the same value in zone $z=+$ and $z=-$, one has the identity

$$
\begin{aligned}
& P . V . \int_{s \in I_{+}^{\varepsilon}} \frac{c_{s, p}^{\varepsilon}}{\lambda_{s, p}^{\varepsilon}-\mu} \frac{\exp (-s)}{t_{s}^{\varepsilon}} d s+\frac{\mu}{(2 \pi p)^{2}} P . V . \int_{s \in I_{+}^{\varepsilon}} \frac{c_{s, p}^{\varepsilon}}{1 / t_{s}^{\varepsilon}-\mathbf{i} \mu / 2 \pi p} \exp (-s) d s \\
& =\frac{\mathbf{i}}{2 \pi p} P . V . \int_{s \in I_{+}^{\varepsilon}} \frac{c_{s, p}^{\varepsilon}}{\lambda_{s, p}^{\varepsilon}-\mu} \exp (-s)\left(\lambda_{s, p}^{\varepsilon}-\mu\right) d s=\frac{\mathbf{i}}{2 \pi p} \int_{s \in I_{+}^{\varepsilon}} c_{s, p}^{\varepsilon} \exp (-s) d s .
\end{aligned}
$$


Similarly

$P . V . \int_{s \in I_{-}^{\varepsilon}} \frac{c_{s, p}^{\varepsilon}}{\lambda_{s,-p}^{\varepsilon}-\mu} \frac{\exp (-s)}{t_{s}^{\varepsilon}} d s-\frac{\mu}{(2 \pi p)^{2}} P . V . \int_{s \in I_{-}^{\varepsilon}} \frac{c_{s, p}^{\varepsilon}}{-1 / t_{s}^{\varepsilon}-\mathbf{i} \mu / 2 \pi p} \exp (-s) d s$
$=\frac{-\mathbf{i}}{2 \pi p} P . V . \int_{s \in I_{-}^{\varepsilon}} \frac{c_{s, p}^{\varepsilon}}{\lambda_{s,-p}^{\varepsilon}-\mu} \exp (-s)\left(\lambda_{s,-p}^{\varepsilon}-\mu\right) d s=\frac{-\mathbf{i}}{2 \pi p} \int_{s \in I_{-}^{\varepsilon}} c_{s, p}^{\varepsilon} \exp (-s) d s$.

The sum of these two identities yields the results because $I_{+}^{\varepsilon}=I_{-}^{\varepsilon}$ and the proof is ended.

Lemma 5.2. Let $\mu \in \mathbf{i} \mathbb{R}^{*}$ and $p \neq 0$. The function $f_{a, b, p}^{\varepsilon}$ is a Hilbert transform

$$
f_{a, b, p}^{\varepsilon}(\mu)=-P . V \cdot \int_{\mathbb{R}} \frac{m_{a, b, p}^{\varepsilon}(\lambda)}{\lambda-\mathbf{i} \mu} d \lambda
$$

where the kernel is

$$
m_{a, b, p}^{\varepsilon}(\lambda)=\left(a, v_{s^{\varepsilon}(|\lambda|), p, \operatorname{sign}(\lambda)}^{\varepsilon}\right)\left(v_{s^{\varepsilon}(|\lambda|), p, \operatorname{sign}(\lambda)}^{\varepsilon}, b\right) \exp \left(-s^{\varepsilon}(|\lambda|)\right)\left(s^{\varepsilon}\right)^{\prime}(|\lambda|) .
$$

Moreover the kernel is uniformly square integrable: there exists a constant $C>0$ (independent of $p$ and $\varepsilon \in(0,1])$ such that $\left\|m_{a, b, p}^{\varepsilon}\right\|_{L^{2}(\mathbb{R})} \leq C\|a\|_{L^{2}(I)}\|b\|_{L^{2}(I)}$.

Proof. The kernel $m_{a, b, p}^{\varepsilon}$ is just a convenient compact way to recast (5.4) in combination with (3.13) the change of variable $s=s^{\varepsilon}(\lambda)=\varepsilon^{2} s(\lambda / \varepsilon)$. One has $\left\|m_{a, b, p}^{\varepsilon}\right\|_{L^{2}(\mathbb{R})}^{2} \leq \int_{\mathbb{R}}\left(\|a\|_{L^{2}(I)}^{2}\|b\|_{L^{2}(I)}^{2} \exp \left(-2 s^{\varepsilon}(|\lambda|)\right)\left(s^{\varepsilon}\right)^{\prime}(|\lambda|)^{2}\right) d \lambda$ that is $\left\|m_{a, b, p}^{\varepsilon}\right\|_{L^{2}(\mathbb{R})}^{2} \leq 2\|a\|_{L^{2}(I)}^{2}\|b\|_{L^{2}(I)}^{2} i(\varepsilon)$. One has that the integral $i(\varepsilon)=\int_{\mathbb{R}^{+}} e^{-2 \varepsilon^{2} s(\lambda / \varepsilon)} \varepsilon^{2} s^{\prime}(\lambda / \varepsilon)^{2} d \lambda$ is continuously defined for $\varepsilon \in(0,1]$. By means of the Lebesgue dominated convergence theorem and Proposition 3.8, one has $\lim _{\varepsilon \rightarrow 0^{+}} i(\varepsilon)=\int_{\mathbb{R}^{+}} \exp \left(-\lambda^{2} / 2\right) \lambda^{2} d \lambda$. So there exists a constant $C$ such that $i(\varepsilon) \leq C$ for all $\varepsilon \in(0,1]$. It ends the proof.

Proposition 5.3. There exists a constant $C>0$ (independent of $p \neq 0$ and $0<\varepsilon \leq 1)$ such that

$$
\left\|\left(m_{a, b, p}^{\varepsilon}\right)^{\prime}\right\|_{L^{2}(\mathbb{R})} \leq C|p|\|a\|_{L^{2}(I)}\|b\|_{L^{2}(I)} .
$$

Proof. The calculations are given for $\lambda>0$. By definition $v_{s^{\varepsilon}(\lambda), p, \operatorname{sign}^{\varepsilon}(\lambda)}(x)=$ $\exp \left(-2 \mathbf{i} \pi p \lambda \int_{0}^{x} \frac{d t}{\mu_{s(\lambda)}^{\varepsilon}(t)}\right)$ so

$$
\begin{gathered}
\frac{d}{d \lambda} v_{s^{\varepsilon}(\lambda), p, \operatorname{sign}(\lambda)}^{\varepsilon}(x)=-2 \mathbf{i} \pi p \exp \left(-2 \mathbf{i} \pi p \lambda \int_{0}^{x} \frac{d t}{\mu_{s^{\varepsilon}(\lambda)}^{\varepsilon}(t)}\right) \\
\left(\int_{0}^{x} \mu_{s^{\varepsilon}(\lambda)}^{\varepsilon}(t)^{-1} d t+\lambda\left(s^{\varepsilon}\right)^{\prime}(\lambda) \frac{d}{d s} \int_{0}^{x} \mu_{s^{\varepsilon}(\lambda)}^{\varepsilon}(t)^{-1} d t\right) .
\end{gathered}
$$


Recall that $1 / \lambda=t_{s}^{\varepsilon}=\int_{0}^{1} \mu_{s^{\varepsilon}(\lambda)}^{\varepsilon}(t)^{-1} d t$. So $\int_{0}^{x} \mu_{s^{\varepsilon}(\lambda)}^{\varepsilon}(t)^{-1} d t \leq 1 / \lambda$. Moreover

$$
-1 / \lambda^{2}=\left.\left(s^{\varepsilon}\right)^{\prime}(\lambda) \frac{d}{d s} \int_{0}^{1} \mu_{s^{\varepsilon}}^{\varepsilon}(t)^{-1} d t\right|_{s=s^{\varepsilon}(\lambda)} .
$$

Since $\frac{d}{d s} \mu_{s^{\varepsilon}(\lambda)}^{\varepsilon}(t)<0$ for all $0<t<1$, one gets that $\left|\left(s^{\varepsilon}\right)^{\prime}(\lambda) \frac{d}{d s} \int_{0}^{x} \mu_{s^{\varepsilon}(\lambda)}^{\varepsilon}(t)^{-1} d t\right| \leq$ $\left|\left(s^{\varepsilon}\right)^{\prime}(\lambda) \frac{d}{d s} \int_{0}^{1} \mu_{s^{\varepsilon}(\lambda)}^{\varepsilon}(t)^{-1}\right| \leq 1 / \lambda^{2}$. Therefore $\left|\frac{d}{d \lambda} v_{s^{\varepsilon}(\lambda), p, \operatorname{sign}(\lambda)}^{\varepsilon}(x)\right| \leq \frac{4 \pi|p|}{|\lambda|}$. Since $m_{a, b, p}(5.5)$ is the product of 4 terms, the derivative can be expressed as the sum of 4 contributions. In view of (5.5) one gets

$$
\begin{aligned}
& \left\|\frac{d}{d \lambda} m_{a, b, p}^{\varepsilon}(\lambda)\right\|_{L^{2}(\mathbb{R})} \\
& \leq 4 \pi|p|\|a\|_{L^{2}(I)} \quad \times \quad\|b\|_{L^{2}(I)} \quad \times \quad\left(\int_{\mathbb{R}} \frac{1}{\lambda^{2}} \exp \left(-2 s^{\varepsilon}(|\lambda|)\right)\left(s^{\varepsilon}\right)^{\prime}(|\lambda|)^{2} d \lambda\right)^{\frac{1}{2}} \\
& +\|a\|_{L^{2}(I)} \quad \times 4 \pi|p|\|b\|_{L^{2}(I)} \times\left(\int_{\mathbb{R}} \frac{1}{\lambda^{2}} \exp \left(-2 s^{\varepsilon}(|\lambda|)\right)\left(s^{\varepsilon}\right)^{\prime}(|\lambda|)^{2} d \lambda\right)^{\frac{1}{2}} \\
& +\|a\|_{L^{2}(I)} \quad \times \quad\|b\|_{L^{2}(I)} \quad \times \quad\left(\int_{\mathbb{R}} \exp \left(-2 s^{\varepsilon}(|\lambda|)\right)\left(s^{\varepsilon}\right)^{\prime}(|\lambda|)^{4} d \lambda\right)^{\frac{1}{2}} \\
& +\|a\|_{L^{2}(I)} \quad \times \quad\|b\|_{L^{2}(I)} \quad \times\left(\int_{\mathbb{R}} \exp \left(-2 s^{\varepsilon}(|\lambda|)\right)\left(s^{\varepsilon}\right)^{\prime \prime}(|\lambda|)^{2} d \lambda\right)^{\frac{1}{2}} .
\end{aligned}
$$

By a method similar to the one of Lemma 5.2, all integrals in parenthesis are naturally bounded uniformly with respect to $\varepsilon \in(0,1]$. The proof is ended.

5.1.2. Properties of $G_{a, b, p}^{\varepsilon}(\mu)$. The $G_{a, b, p}^{\varepsilon}(\mu)$ correspond to the region of trapped particles. Some of their properties are easy to obtain, but the last one Proposition 5.6 is much more technical because the differentiability of some Abel integrals is delicate: in this work they are characterized in Hölderian norm, see Appendix B.

Lemma 5.4. Let $\mu \in \mathbf{i} \mathbb{R}^{*}$ and $p \neq 0$. One has $G_{a, b, p}^{\varepsilon}(\mu)=\frac{\mu}{(2 \pi p)^{2}} g_{a, b, p}^{\varepsilon}\left(\frac{\mu}{2 \pi p}\right)$ where

$$
\begin{gathered}
g_{a, b, p}^{\varepsilon}(\mu)=-P . V \cdot \int_{s \in I_{c}^{\varepsilon}} \frac{\left(a, v_{s, p, c}^{\varepsilon}\right)\left(v_{s, p, c}^{\varepsilon}, b\right)}{1 / t_{s}^{\varepsilon}-i \mu} \exp (-s) d s \\
\quad+P . V . \int_{s \in I_{c}^{\varepsilon}} \frac{\left(a, v_{s,-p, c}^{\varepsilon}\right)\left(v_{s,-p, c}^{\varepsilon}, b\right)}{-1 / t_{s}^{\varepsilon}-i \mu} \exp (-s) d s .
\end{gathered}
$$

Proof. The proof is fundamentally the same as the one of Lemma 5.1. It uses the identity $\left(a, v_{s, p, c}^{\varepsilon}\right)\left(v_{s, p, c}^{\varepsilon}, b\right)=\left(a, v_{s,-p, c}^{\varepsilon}\right)\left(v_{s,-p, c}^{\varepsilon}, b\right)$.

Lemma 5.5. Let $\mu \in \mathbf{i} \mathbb{R}^{*}$ and $p \neq 0$. The function $g_{a, b, p}^{\varepsilon}$ is a Hilbert transform

$$
g_{a, b, p}^{\varepsilon}(\mu)=-P . V \cdot \int_{\mathbb{R}} \frac{n_{a, b, p}^{\varepsilon}(\lambda)}{\lambda-\mathbf{i} \mu} d \lambda
$$


where the kernel $n_{a, b, p}^{\varepsilon}(\lambda)$ is for $\lambda \in[-\varepsilon \mathcal{B}, \varepsilon \mathcal{B}]$

$$
n_{a, b, p}^{\varepsilon}(\lambda)=\left(a, v_{s_{c}^{\varepsilon}(|\lambda|), \operatorname{sign}(\lambda) p, c}^{\varepsilon}\right)\left(v_{s_{c}^{\varepsilon}(|\lambda|), \operatorname{sign}(\lambda) p, c}^{\varepsilon}, b\right) \exp \left(-s_{c}^{\varepsilon}(|\lambda|)\right)\left(s_{c}^{\varepsilon}\right)^{\prime}(|\lambda|)
$$

and $n_{a, b, p}^{\varepsilon}(\lambda)=0$ for $\lambda \notin[-\varepsilon \mathcal{B}, \varepsilon \mathcal{B}]$. Moreover the kernel is uniformly square integrable: there exists $C>0$ (independent of $p$ and $\varepsilon \in(0,1])$ such that $\left\|n_{a, b, p}^{\varepsilon}\right\|_{L^{2}(\mathbb{R})} \leq C \sqrt{\varepsilon}\|a\|_{L^{2}(I)}\|b\|_{L^{2}(I)}$.

Proof. The boundedness is as follows

$$
\begin{gathered}
\left\|n_{a, b, p}^{\varepsilon}\right\|_{L^{2}(\mathbb{R})}^{2} \leq \int_{\mathbb{R}}\left(\|a\|_{L^{2}(I)}^{2}\|b\|_{L^{2}(I)}^{2} \exp \left(-2 s_{c}^{\varepsilon}(|\lambda|)\right)\left(s_{c}^{\varepsilon}\right)^{\prime}(|\lambda|)^{2}\right) d \lambda \\
\leq 2\|a\|_{L^{2}(I)}^{2}\|b\|_{L^{2}(I)}^{2} j(\varepsilon)
\end{gathered}
$$

where the integral $j(\varepsilon)=\int_{\mathbb{R}}\left(s_{c}^{\varepsilon}\right)^{\prime}(\lambda)^{2} d \lambda=\varepsilon^{2} \int_{\mathbb{R}} s_{c}^{\prime}(\lambda / \varepsilon)^{2} d \lambda=\varepsilon \int_{\mathbb{R}} s_{c}^{\prime}(\lambda)^{2} d \lambda \leq$ $C \varepsilon$. The proof is ended.

The important technical result which gathers most of the difficulties of this part is the following. Indeed for reasons explained in Appendix B, one cannot calculate a full derivative of $n_{a, b, p}^{\varepsilon}$.

Proposition 5.6. Let $p \neq 0$ and $0<\varepsilon \leq 1$. One has the Hölder-continuity $\left\|n_{a, b, p}^{\varepsilon}\right\|_{C^{0,1 / 2(\mathbb{R})}} \leq C|p| \sqrt{\varepsilon}\|a\|_{L^{2}(I)}\|b\|_{L^{2}(I)}$.

Proof. Using the rescaled quantities (3.19), one can write $n_{a, b, p}^{\varepsilon}(\lambda)=\varepsilon Q(\lambda / \varepsilon)$ with

$$
Q(\lambda)=\underbrace{\left(a, v_{\varepsilon^{2} s_{c}(|\lambda|), \operatorname{sign}(\lambda) p, c}^{\varepsilon}\right)}_{=\widehat{f}(\lambda)} \underbrace{\left(v_{\varepsilon^{2} s_{c}(|\lambda|), \operatorname{sign}(\lambda) p, c}^{\varepsilon}, b\right)}_{=\widehat{g}(\lambda)} \underbrace{\exp \left(-\varepsilon^{2} s_{c}(|\lambda|)\right) s_{c}^{\prime}(|\lambda|)}_{=h(\lambda)}
$$

for $\lambda \in[-\mathcal{B}, \mathcal{B}]$. The function $\widehat{f}(\lambda)$ is studied in Appendix $\mathrm{B}, \widehat{g}(\lambda)$ has similar properties and $h(\lambda)$ is known thanks to Proposition 3.10. For $0<\lambda<\mu<\mathcal{B}$, one has the telescopic decomposition

$Q(\lambda)-Q(\mu)=(f(\lambda)-f(\mu)) g(\lambda) h(\lambda)+(g(\lambda)-g(\mu)) f(\mu) h(\lambda)+f(\mu) g(\mu)(h(\lambda)-h(\mu))$.

Proposition B.1 yields

$$
\begin{aligned}
& |Q(\lambda)-Q(\mu)| \\
& \leq C|p|\|a\|_{L^{2}(I)} \frac{\sqrt{|\lambda-\mu|}}{\sqrt{\min \left(\varphi_{0}^{\prime}\left(\widehat{a}_{s_{c}(\lambda)}\right), \varphi_{0}^{\prime}\left(\widehat{b}_{s_{c}(\lambda)}\right)\right)}} \times C\|b\|_{L^{2}(I)} \times\left|s_{c}^{\prime}(\lambda)\right| \\
& +C|p|\|b\|_{L^{2}(I)} \frac{\sqrt{|\lambda-\mu|}}{\sqrt{\min \left(\varphi_{0}^{\prime}\left(\widehat{a}_{s_{c}(\lambda)}\right), \varphi_{0}^{\prime}\left(\widehat{b}_{s_{c}(\lambda)}\right)\right)}} \times C\|a\|_{L^{2}(I)} \times\left|s_{c}^{\prime}(\lambda)\right| \\
& +C\|a\|_{L^{2}(I)} \times C\|b\|_{L^{2}(I)} \times|\lambda-\mu|\left\|s_{c}^{\prime}\right\|_{W^{1, \infty}} .
\end{aligned}
$$

It appears that $\frac{1}{\sqrt{\min \left(\varphi_{0}^{\prime}\left(\widehat{a}_{s_{c}(\lambda)}\right), \varphi_{0}^{\prime}\left(\widehat{b}_{s_{c}(\lambda)}\right)\right)}}\left|s_{c}^{\prime}(\lambda)\right| \in L^{\infty}(0, \mathcal{B})$ is view of Proposition 3.10 and the rough bounds (B.2-B.3). One gets that $Q \in C^{0,1 / 2}[0, \mathcal{B}]$ is Hölder, with a continuity constant $C|p|\|a\|_{L^{2}(I)}\|p\|_{L^{2}(I)}$. Since $Q$ is extended by continuity by 0 outside $[\mathcal{B}, \infty)$, then $Q \in C^{0,1 / 2}\left(\mathbb{R}^{+}\right)$is Hölder 
over $\mathbb{R}^{+}$with the same norm. Additionally $Q$ is continuous at $\lambda=0$ and is also $Q \in C^{0,1 / 2}\left(\mathbb{R}^{-}\right)$. So $Q \in C^{0,1 / 2}(\mathbb{R})$. Same property holds for $n_{a, b, p}^{\varepsilon}$ with a rescaling parameter by $\sqrt{\varepsilon}$. The proof is ended.

5.1.3. Well posedness of the variational Lippmann-Schwinger equation. After elimination of $F_{a, b, p}^{\varepsilon}(\mu)$ and $G_{a, b, p}^{\varepsilon}(\mu)$ in function of $f_{a, b, p}^{\varepsilon}(\mu)$ and $g_{a, b, p}^{\varepsilon}(\mu)$ and division by $\mu$ which is non zero, the variational Lippmann-Schwinger equation (5.3) admits another formulation.

Find $a \in L_{0}^{2}(I)$ such that for all $b \in L_{0}^{2}(I)$

$$
(a, b)+\sum_{p \neq 0} \frac{\varepsilon^{2}}{4 p^{2} \pi^{2}} f_{a, b, p}^{\varepsilon}\left(\frac{\mu}{2 \pi p}\right)+\sum_{p>0} \frac{\varepsilon^{2}}{4 p^{2} \pi^{2}} g_{a, b, p}^{\varepsilon}\left(\frac{\mu}{2 \pi p}\right)=\frac{\varepsilon}{\mu} l^{\varepsilon}(b) .
$$

It is clear that the terms $\frac{1}{p^{2}}$ are amenable for the convergence of the series. It remains to estimate the other terms. It poses no real difficulty for the terms $f_{a, b, p}^{\varepsilon}$, but needs a little more technical efforts for the terms $g_{a, b, p}^{\varepsilon}$ which correspond to the region of trapped particles.

The $L^{2}$-continuity of the Hilbert transform yields

$$
\left\|f_{a, b, p}^{\varepsilon}\right\|_{L^{2}(\mathbb{R})}=\pi\left\|m_{a, b, p}^{\varepsilon}\right\|_{L^{2}(\mathbb{R})} \leq C\|a\|_{L^{2}(I)}\|b\|_{L^{2}(I)}
$$

and

$$
\left\|\left(f_{a, b, p}^{\varepsilon}\right)^{\prime}\right\|_{L^{2}(\mathbb{R})}=\pi\left\|\left(m_{a, b, p}^{\varepsilon}\right)^{\prime}\right\|_{L^{2}(\mathbb{R})} \leq C|p|\|a\|_{L^{2}(I)}\|b\|_{L^{2}(I)} .
$$

By Sobolev imbedding and interpolation

$$
\left\|f_{a, b, p}^{\varepsilon}\right\|_{L^{\infty}(\mathbb{R})} \leq c\left\|f_{a, b, p}^{\varepsilon}\right\|_{H^{\frac{3}{4}(\mathbb{R})}} \leq c\left\|f_{a, b, p}^{\varepsilon}\right\|_{L^{2}(\mathbb{R})}^{\frac{1}{4}}\left\|\left(f_{a, b, p}^{\varepsilon}\right)^{\prime}\right\|_{L^{2}(\mathbb{R})}^{\frac{3}{4}} \leq \widehat{c}|p|^{\frac{3}{4}}\|a\|_{L^{2}(I)}\|b\|_{L^{2}(I)} .
$$

Therefore

$$
\sum_{p \neq 0} \frac{1}{p^{2}}\left\|f_{a, b, p}^{\varepsilon}\right\|_{L^{\infty}(\mathbb{R})} \leq C\left(\sum_{p \geq 1} \frac{1}{p^{5 / 4}}\right) c_{\varphi}\|a\|_{L^{2}(I)}\|b\|_{L^{2}(I)} \leq d\|a\|_{L^{2}(I)}\|b\|_{L^{2}(I)} .
$$

The other terms are bounded as follows. The length of the support of $n_{a, b, p}^{\varepsilon}$ is equal to $\varepsilon \mathcal{B}$. One has the bound $\left\|n_{a, b, p}^{\varepsilon}\right\|_{L^{\infty}(\mathbb{R})} \leq C \varepsilon\|a\|_{L^{2}(I)}\|b\|_{L^{2}(I)} \leq$ $C \sqrt{\varepsilon}\|a\|_{L^{2}(I)}\|b\|_{L^{2}(I)}$. One has the decomposition $g_{a, b, p}^{\varepsilon}(\mu)=\int_{-\infty}^{\mu-h} \frac{n_{a, b, p}^{\varepsilon}(\lambda)}{\lambda-\mu} d \lambda+$ $\int_{\mu-h}^{\mu+h} \frac{n_{a, b, p}^{\varepsilon}(\lambda)-n_{a, b, p}^{\varepsilon}(\mu)}{\lambda-\mu} d \lambda+\int_{\mu+h}^{\infty} \frac{n_{a, b, p}^{\varepsilon}(\lambda)}{\lambda-\mu} d \lambda$. So

$$
\begin{gathered}
\left|g_{a, b, p}^{\varepsilon}(\mu)\right| \leq\left\|n_{a, b, p}^{\varepsilon}\right\|_{L^{\infty}(\mathbb{R})} \int_{\mu-h-\mathcal{B}}^{\mu-h} \frac{1}{\lambda-\mu} d \lambda \\
+\left\|n_{a, b, p}^{\varepsilon}\right\|_{C^{0,1 / 2}(\mathbb{R})} \int_{\mu-h}^{\mu+h} \frac{1}{|\lambda-\mu|^{\frac{1}{2}}} d \lambda+\left\|n_{a, b, p}^{\varepsilon}\right\|_{L^{\infty}(\mathbb{R})} \int_{\mu+h}^{\mu+h+\mathcal{B}} \frac{1}{\lambda-\mu} d \lambda \\
\leq 2\left\|n_{a, b, p}^{\varepsilon}\right\|_{L^{\infty}(\mathbb{R})} \log (1+\mathcal{B} / h)+4\left\|n_{a, b, p}^{\varepsilon}\right\|_{C^{0,1 / 2}(\mathbb{R})} \sqrt{h} .
\end{gathered}
$$


Let us take $h=B / p^{2}$ for $p \geq 1$. One gets $\left|g_{a, b, p}^{\varepsilon}(\mu)\right| \leq C \sqrt{\varepsilon}(1+\log |p|)\|a\|_{L^{2}(I)}\|b\|_{L^{2}(I)}$. It holds for all $\mu \in \mathbb{R}$ so $\left\|g_{a, b, p}^{\varepsilon}(\mu)\right\|_{L^{\infty}(\mathbb{R})} \leq C \sqrt{\varepsilon}(1+\log |p|)\|a\|_{L^{2}(I)}\|b\|_{L^{2}(I)}$. The $\log |p|$ is now sufficient for the convergence of the series

$$
\begin{aligned}
\sum_{p \neq 0} \frac{1}{p^{2}}\left\|g_{a, b, p}^{\varepsilon}\right\|_{L^{\infty}(\mathbb{R})} & \leq C \sqrt{\varepsilon}\left(\sum_{p \geq 1} \frac{1+\log p}{p^{2}}\right) c_{\varphi}\|a\|_{L^{2}(I)}\|b\|_{L^{2}(I)} \\
& \leq D \sqrt{\varepsilon}\|a\|_{L^{2}(I)}\|b\|_{L^{2}(I)} .
\end{aligned}
$$

With the above material, it is easy to formulate and prove the

Theorem 5.7. There exists $\varepsilon_{*}>0$ such that: for $0<\varepsilon<\varepsilon_{*}$, the variational problem (5.7) is coercive for all values of $z \in\{+,-, c\}, k \neq 0$ and $e \in I_{z}^{\varepsilon}$. The unique solution is denoted as $a_{e, k, z}^{\varepsilon}$ and there exists a constant $C>0$ (which depends solely on $\varphi_{0}$ ) such that

$$
\left\|a_{e, k, z}^{\varepsilon}\right\|_{L^{2}(I)} \leq C \frac{\varepsilon \exp (-e / 2)}{|k|}, \quad k \neq 0 .
$$

Proof. The bilinear form (5.7) on the left and side of the variational problem (5.3) is coercive provided $\varepsilon$ is less that some positive threshold: this is uniform with respect to $\mu$. The rest of the proof is evident: let $C$ be an upper bound of the inverse of the bilinear form; the bound (5.9) comes from

$$
\left\|a_{e, k, z}^{\varepsilon}\right\|_{L^{2}(I)} \leq C \varepsilon / \mu\left\|l^{\varepsilon}(b)\right\|_{L^{2}(I)^{*}} \leq C \frac{\varepsilon}{\left|\lambda_{e, k}^{\varepsilon}\right|} \frac{2 \exp (-e / 2)}{t_{e}^{\varepsilon}} \leq \frac{C \varepsilon \exp (-e / 2)}{\pi|k|} .
$$

The interpretation of the summability in (4.6) needs a comment. Indeed the algebra of Lemma 5.1 shows that $F_{a, b, p}^{\varepsilon}$ is obtained from (4.6) as the sum of two terms which are individually a convergent principal value proportional to $p^{-2}$ (which poses no problem) plus a simple integral proportional to $p^{-1}$. These simple integrals yield a series which is not studied in this work. Actually a simple interpretation is possible in the case $\varphi_{0}=0$ or $\varepsilon=0$. In this case, $v_{s, p,+}^{\varepsilon}(x)=v_{s,-p,-}^{\varepsilon}(x)=\exp (2 \mathbf{i} \pi p x)$ are both equal to the same Fourier mode.

Remark 5.8 (Interpretation of the double sums). The above discussion shows that double sums like $\sum_{z^{\prime} \in+,-, c} \sum_{p \neq 0} \gamma_{p, z^{\prime}}$ should be interpreted after rearrangement as $\sum_{p \neq 0}\left(\gamma_{p,+}+\gamma_{-p,-}\right)+\sum_{p>0}\left(\gamma_{p, c}+\gamma_{-p, c}\right)$.

\subsection{Definition the $V_{e, k, z}^{\varepsilon}$}

Now that $a_{e, k, z}^{\varepsilon}$ is defined for $k \neq 0$, one plugs in (4.4) which is now written as $V_{e, k, z}^{\varepsilon}(x)=U_{e, k, z}^{\varepsilon}(x)+a_{e, k, z}^{\varepsilon}(x) e_{0}+R_{e, k, z}^{\varepsilon}(x)$ where

$$
R_{e, k, z}^{\varepsilon}(x)=\varepsilon \sum_{z^{\prime}} \sum_{p \neq 0} P . V . \int_{s \in I_{z^{\prime}}^{\varepsilon}} R_{\substack{s, p, z^{\prime} \\ e, k, z}}^{\varepsilon}(x) t_{s}^{\varepsilon} d s
$$


with $\quad R_{s, p, z^{\prime}}^{\varepsilon}(x)=\frac{\left(\alpha a_{e, k, z}^{\varepsilon} e_{2}, \exp \left(\varepsilon^{2} \varphi_{0} / 2\right) U_{s, p, z^{\prime}}^{\varepsilon}\right)}{\lambda_{s, p}^{\varepsilon}-\lambda_{e, k}^{\varepsilon}} U_{s, p, z^{\prime}}^{\varepsilon}(x)$. The weak form definition of $V_{e, k, z}^{e, k}$ writes

$$
\begin{aligned}
& \left(V_{e, k, z}^{\varepsilon}, U\right)=\left(U_{e, k, z}^{\varepsilon}, U\right)+\left(a_{e, k, z}^{\varepsilon}(x) e_{0}, U\right) \\
& +\varepsilon \sum_{z^{\prime}} \sum_{p \neq 0} P . V . \int_{s \in I_{z^{\prime}}^{\varepsilon}} \frac{\left(\alpha a_{e, k, z}^{\varepsilon} e_{2}, \exp \left(\varepsilon^{2} \varphi_{0} / 2\right) U_{s, p, z^{\prime}}^{\varepsilon}\right)}{\lambda_{s, p}^{\varepsilon}-\lambda_{e, k}^{\varepsilon}}\left(U_{s, p, z^{\prime}}^{\varepsilon}, U\right) t_{s}^{\varepsilon} d s .
\end{aligned}
$$

Another notation is the following. For $p \neq 0$ define the integrals

$$
\underset{\bar{F}_{p}^{\varepsilon}, k, z}{\varepsilon}=P . V . \int_{s \in I_{+}^{\varepsilon}}\left(\begin{array}{c}
R_{s, p,+}^{\varepsilon, k, z} \\
e, U
\end{array}\right) t_{s}^{\varepsilon} d s+P . V . \int_{s \in I_{-}^{\varepsilon}}\left(\begin{array}{c}
R_{s,-p,-}^{\varepsilon}, U \\
e, k, z
\end{array}\right) t_{s}^{\varepsilon} d s
$$

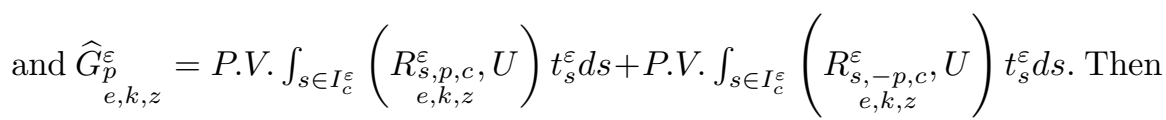
the formula (5.10-5.11) yield the identity

$$
\left(V_{e, k, z}^{\varepsilon}-U_{e, k, z}^{\varepsilon}, U\right)=\left(a_{e, k, z}^{\varepsilon}(x) e_{0}, U\right)+\varepsilon \sum_{p \neq 0} \widehat{F}_{e, k, z}^{\varepsilon}(\lambda)+\varepsilon \sum_{p \neq 0} \widehat{G}_{e, k, z}^{\varepsilon}(\lambda)
$$

with the notation $e=s_{z}^{\varepsilon}(\lambda)$. The terms in the right hand side of the expansion (5.12) are studied in way which allows to study the norm (5.14).

The integrability is interpreted as a principal value with the same method as in the previous section, using also that $\left(U_{s, p, z^{\prime}}^{\varepsilon}, U\right)$ is well defined since $U_{s, p, z^{\prime}}^{\varepsilon} \in L^{1}(I)^{\mathbb{N}}$ for all $s, p, z^{\prime}$ and $U$ has a finite number of non zero components, all of them in $L^{\infty}(I)$. The summability can be established with the same methods as in the previous section (taking $U \in X_{0}$ greatly simplifies this issue). Looking carefully to the Lippmann-Schwinger equation (4.6), an immediate result for the third component of the vector $V_{e, k, z}^{\varepsilon}$ is that $V_{e, k, z}^{\varepsilon} \cdot e_{2}=\frac{1}{\varepsilon \alpha} \exp \left(-\varepsilon^{2} \varphi_{0} / 2\right) \lambda_{e, k}^{\varepsilon} a_{e, k, z}^{\varepsilon} \in L^{2}(I)$.

\subsection{Completness the $V_{e, k, z}^{\varepsilon}$}

We concentrate on proving that the whole family $\left(V_{e, k, z}^{\varepsilon}\right)_{k \neq 0}$ completed by the vectors in the null space defined in (4.2) an (4.3) is a Hilbert basis in $X$. For a scalar function $q$ defined for $e \in I_{z}^{\varepsilon}$, we denote the weighted $L^{2}$ norm as

$$
|q|_{z}^{\varepsilon}=\left(\int_{I_{z}^{\varepsilon}}|q(e)|^{2} t_{e}^{\varepsilon} d e\right)^{\frac{1}{2}}
$$

Let us define the norm

$$
\||U|\|^{\varepsilon}=\left(\sum_{k \neq 0}\left|\left(U, V_{k}^{\varepsilon}\right)\right|^{2}+\sum_{z} \sum_{k}\left(\left|\left(U, V_{e, k, z}^{\varepsilon}\right)\right|_{z}^{\varepsilon}\right)^{2}\right)^{\frac{1}{2}}
$$


where one reintroduces the vectors in the null space which are: $V_{e, 0, z}^{\varepsilon}$ for all possible $e$ and $z$ defined in (4.3); and $V_{k}^{\varepsilon}=V_{\exp (2 i \pi k x)}^{\varepsilon}$ defined in (4.2) for $k \in \mathbb{Z}^{*}$. Since the norm $\left.\||U|\|\right|^{\varepsilon}$ is the square root of the sum of non negative terms, there is no need to reorganize the sums as in Remark 5.8. We also define the semi-norm

$|U|^{\varepsilon}=\left(\|U\|^{2}-\sum_{z} \int_{e \in I_{z}^{\varepsilon}}\left(\left|\left(U, U_{e, 0, z}^{\varepsilon}\right)\right|_{z}^{\varepsilon}\right)^{2} t_{e}^{\varepsilon} d e\right)^{\frac{1}{2}}$. The completeness is a consequence of the following result.

Theorem 5.9. There exists a constant $C>0$ independent of $\varepsilon$ such that $\|\mid U\|\left\|^{\varepsilon} \leq(1+C \varepsilon)\right\| U \|$ for all $U \in X$. Moreover there exists $\varepsilon_{*}>0$ such that $\|U\| \leq(1+C \varepsilon)\|\| U \|\left.\right|^{\varepsilon}$ for all $U \in X$ and $0<\varepsilon \leq \varepsilon_{*}$.

Proof. The proof is established at the end of Section 5.3.4, after proving various estimates in Sections 5.3.1 to 5.3.3.

5.3.1. A preliminary manipulation. For convenience, a unification of notations is possible by taking $s_{+}^{\varepsilon}=s_{-}^{\varepsilon}=s^{\varepsilon}$. We extend by continuity the functions $s_{+}^{\varepsilon}=s_{-}^{\varepsilon}$ by 0 so that

$$
\left(s_{+}^{\varepsilon}\right)^{\prime}(\lambda)=\left(s_{-}^{\varepsilon}\right)^{\prime}(\lambda)=0 \text { for } \lambda \leq 0 .
$$

We also extend by continuity the function $s_{z}^{\varepsilon}$ by constants so that $\left(s_{c}^{\varepsilon}\right)^{\prime}(\lambda)=0$ for $\lambda \leq 0$ or $\lambda \geq \varepsilon \mathcal{B}$. Now $s_{z}^{\varepsilon} \in C^{1}(\mathbb{R})$ for all $z$.

Lemma 5.10. One can write

$$
\widehat{F}_{e, k, z}^{\varepsilon}=\int_{x \in I} f(\lambda, x)\left(\frac{1}{\pi} P . V \cdot \int_{\mu \in \mathbb{R}} \frac{\mu g(\mu, x)}{2 \mathbf{i} \pi k \lambda-2 \mathbf{i} p \mu} d \mu\right) d x
$$

where $f$ and $g$ are defined in the proof. A similar reformulation holds for $\widehat{G}_{p}^{\varepsilon} \quad$ after convenient redefinition of $g$. $e, k, z$

Proof. Use (5.10) and (5.1), denote $e=s_{z}^{\varepsilon}(\lambda)$ and $s=s^{\varepsilon}(\mu)$ and notice the identity $t_{s^{\varepsilon}(\mu)}^{\varepsilon}=\mu^{-1}$. One can write

$$
\begin{gathered}
\left(\begin{array}{c}
\left.R_{s, p,+}^{\varepsilon}, U\right) \\
e, k, z
\end{array}\right) t_{s}^{\varepsilon}=\frac{\left(a_{e, k, z}^{\varepsilon} e_{2}, \alpha t_{s}^{\varepsilon} \exp \left(\varepsilon^{2} \varphi_{0} / 2\right) U_{s, p,+}^{\varepsilon}\right)}{\lambda_{s, p}^{\varepsilon}-\lambda_{e, k}^{\varepsilon}}\left(U_{s, p,+}^{\varepsilon}, U\right) \\
=\int_{x \in I} a_{e, k, z}^{\varepsilon}(x) \frac{\left(U_{s, p,+}^{\varepsilon}, U\right) v_{s, p,+}^{\varepsilon}(x) \exp (-s / 2)}{2 \mathbf{i} \pi p \mu-2 \mathbf{i} \pi k \lambda} d x
\end{gathered}
$$

One obtains after integration with respect to $s \in I_{+}^{\varepsilon}$

$$
\begin{gathered}
P . V \cdot \int_{s \in I_{+}^{\varepsilon}}\left(\begin{array}{c}
\left.R_{s, p,+}^{\varepsilon}, U\right) t_{s}^{\varepsilon} d s \\
e, k, z
\end{array}\right. \\
=\int_{x \in I} a_{e, k, z}^{\varepsilon}(x)\left(P . V \cdot \int_{s \in I_{+}^{\varepsilon}} \frac{\left(U_{s, p,+}^{\varepsilon}, U\right) v_{s, p,+}^{\varepsilon}(x) e^{-s / 2}}{2 \mathbf{i} \pi p \mu-2 \mathbf{i} \pi k \lambda} d s\right) d x
\end{gathered}
$$


$=\int_{x \in I} a_{e, k, z}^{\varepsilon}(x)\left(P . V . \int_{\mu>0} \frac{\left(U_{s^{\varepsilon}(\mu), p,+}^{\varepsilon}, U\right) v_{s^{\varepsilon}(\mu), p,+}^{\varepsilon}(x) e^{-s^{\varepsilon}(\mu) / 2}\left(s^{\varepsilon}\right)^{\prime}(\mu)}{2 \mathbf{i} \pi p \mu-2 \mathbf{i} \pi k \lambda} d \mu\right) d x$.

Note the the principal value is correctly defined under mild conditions even if $\lambda=0$ since $\left(s^{\varepsilon}\right)^{\prime}(\mu)$ vanishes at exponential rate near $\mu=0$. A similar identity holds for the second term with $s=s^{\varepsilon}(-\mu)$

$$
\begin{gathered}
P . V . \int_{s \in I_{-}^{\varepsilon}}\left(\begin{array}{c}
\left.R_{s,-p,-}^{\varepsilon}, U\right) t_{s}^{\varepsilon} d s \\
e, k, z
\end{array}\right) d x \\
=\int_{x \in I} a_{e, k, z}^{\varepsilon}(x)\left(P . V . \int_{s \in I_{-}^{\varepsilon}} \frac{\left(U_{s,-p,-}^{\varepsilon}, U\right) v_{s,-p,-}^{\varepsilon}(x) e^{-s / 2}}{2 \mathbf{i} \pi p \mu-2 \mathbf{i} \pi k \lambda} d s\right) d x \\
=\int_{x \in I} a_{e, k, z}^{\varepsilon}(x)(P . V . \\
\left.\int_{\mu<0} \frac{\left(U_{s^{\varepsilon}(-\mu),-p,-}^{\varepsilon}, U\right) v_{s^{\varepsilon}(-\mu),-p,-}^{\varepsilon}(x) e^{\left.-s^{\varepsilon}(-\mu)\right) / 2}\left(s^{\varepsilon}\right)^{\prime}(-\mu)}{2 \mathbf{i} \pi p \mu-2 \mathbf{i} \pi k \lambda} d \mu\right) d x .
\end{gathered}
$$

One can now add the two terms. Denote $e=s_{z}^{\varepsilon}(|\lambda|)$ and $s=s^{\varepsilon}(|\mu|)$, and define the functions $f(\lambda, x)=a_{e, k, z}^{\varepsilon}(x)$ and

$$
\begin{gathered}
g(\mu, x)=-\pi v_{s^{\varepsilon}(|\mu|), p, \operatorname{sign}(\mu)}^{\varepsilon}(x) \\
\times\left(t_{s^{\varepsilon}(|\mu|)}^{\varepsilon} \operatorname{sign}(\mu) U_{s^{\varepsilon}(|\mu|), p, \operatorname{sign}(\mu)}^{\varepsilon}, U\right) \exp \left(-s^{\varepsilon}(|\mu|) / 2\right)\left(s^{\varepsilon}\right)^{\prime}(|\mu|) .
\end{gathered}
$$

Note that $\mu t_{s^{\varepsilon}(\mu)}^{\varepsilon} \operatorname{sign}(\mu)=1$ so that one can simplify the product $\mu g(\mu, x)$ : this notation is convenient for the next lemma 5.12.

5.3.2. Bounds for large $|k|$. Due to the power $|k|^{-\frac{3}{2}}|p|^{-\frac{1}{2}}$, the following bounds are good ones for large values of $|k|$.

Lemma 5.11. For all $z$, one has the bounds

$\left|\widehat{F}_{p}^{\varepsilon}\right|_{\cdot, k, z}^{\varepsilon} \leq \frac{C \varepsilon}{|k|^{\frac{3}{2}}|p|^{\frac{1}{2}}}\left(\left|\left(U_{s^{\varepsilon}, p,+}^{\varepsilon}, U\right)\right|_{+}^{\varepsilon}+\left|\left(U_{s^{\varepsilon},-p,-}^{\varepsilon}, U\right)\right|_{-}^{\varepsilon}\right)$
and $\left|\widehat{G}_{p, k, z}^{\varepsilon}\right|_{z}^{\varepsilon} \leq \frac{C \varepsilon}{|k|^{\frac{3}{2}}|p|^{\frac{1}{2}}}\left(\left|\left(U_{s_{c}^{\varepsilon}, p, c}^{\varepsilon}, U\right)\right|_{c}^{\varepsilon}+\left|\left(U_{s_{c}^{\varepsilon},-p, c}^{\varepsilon}, U\right)\right|_{c}^{\varepsilon}\right)$.

Proof. For $z=c$, one can gain an extra $\varepsilon^{\frac{1}{2}}$ in the right hand sides. It is sufficient to prove the first estimate. We will make use of the technical inequality of Section C. One has

$$
\left(\left|\widehat{F}_{e, k, z}^{\varepsilon}\right|_{z}^{\varepsilon}\right)^{2}=\left\|\int_{x \in I} \sqrt{t_{s_{z}^{\varepsilon}(|\lambda|)}^{\varepsilon}\left(s_{z}^{\varepsilon}\right)^{\prime}(|\lambda|)} f(\lambda, x)\left(\frac{1}{\pi} P . V \cdot \int_{\mu \in \mathbb{R}} \frac{\mu g(\mu, x)}{2 \mathbf{i} k \lambda-2 \mathbf{i} p \mu} d \mu\right) d x\right\|_{L_{\lambda}^{2}(\mathbb{R})}^{2}
$$

where the integral over $I_{z}^{\varepsilon}$ is extended over $\mathbb{R}$ using (5.15). Using a basic inequality (in Appendix C) and the fact that $\left\|v_{s, p, z}^{\varepsilon}\right\|_{L^{\infty}(I)} \leq 2$, one gets

$$
\left(\left|\widehat{F}_{e, k, z}^{\varepsilon}\right|_{z}^{\varepsilon}\right)^{2} \leq \frac{C}{|k p|} \int_{I}\left\|\sqrt{t_{s_{z}^{\varepsilon}(|\lambda|)}^{\varepsilon}\left(s_{z}^{\varepsilon}\right)^{\prime}(|\lambda|)} a_{e, k, z}^{\varepsilon}(x)\right\|_{L_{\lambda}^{2}(\mathbb{R})}^{2} d x
$$




$$
\times\left\|\left(U_{s^{\varepsilon}(|\lambda|), p, \operatorname{sign}(\lambda)}^{\varepsilon}, U\right) \exp \left(-s^{\varepsilon}(|\lambda|) / 2\right)\left(s^{\varepsilon}\right)^{\prime}(|\lambda|)\right\|_{L_{\lambda}^{2}(\mathbb{R})}^{2} .
$$

The terms on the right hand side are bounded as follows. On the one hand using (5.9)

$$
\begin{gathered}
\int_{I}\left\|\sqrt{t_{s_{z}^{\varepsilon}(|\lambda|)}^{\varepsilon}\left(s_{z}^{\varepsilon}\right)^{\prime}(|\lambda|)} a_{e, k, z}^{\varepsilon}(x)\right\|_{L_{\lambda}^{2}(\mathbb{R})}^{2} d x \\
=\int_{\mathbb{R}}\left\|a_{s_{z}^{\varepsilon}(|\lambda|), k, z}^{\varepsilon}(x)\right\|_{L^{2}(I)}^{2} t_{s_{z}^{\varepsilon}(|\lambda|)}^{\varepsilon}\left(s_{z}^{\varepsilon}\right)^{\prime}(|\lambda|) d \lambda \leq 2 \frac{\varepsilon^{2} \widehat{C}}{|k|^{2}} \int_{\mathbb{R}^{+}} e^{-s_{z}^{\varepsilon}(\lambda)} \frac{1}{\lambda}\left(s_{z}^{\varepsilon}\right)^{\prime}(\lambda) d \lambda .
\end{gathered}
$$

Using (3.20)-(3.21)-(3.22) one has the technical bound for all $z$ and $\varepsilon \in(0,1]$

$$
\int_{\mathbb{R}^{+}} \exp \left(-s_{z}^{\varepsilon}(\lambda)\right) \frac{1}{\lambda}\left(s_{z}^{\varepsilon}\right)^{\prime}(\lambda) d \lambda \leq \bar{C}
$$

For $z=c$, this bound can even be improved $\cdots \leq \varepsilon \bar{C}$. One gets

$$
\int_{I}\left\|\sqrt{t_{s_{z}^{\varepsilon}(|\lambda|)}^{\varepsilon}\left(s_{z}^{\varepsilon}\right)^{\prime}(|\lambda|)} a_{e, k, z}^{\varepsilon}(x)\right\|_{L_{\lambda}^{2}(\mathbb{R})}^{2} d x \leq C \frac{\varepsilon^{2}}{k^{2}} .
$$

One the other hand (3.20)-(3.21)-(3.22) yield also that for some universal constant $C>0 \exp \left(-s^{\varepsilon}(\lambda)\right)\left(s^{\varepsilon}\right)^{\prime}(\lambda) \leq C \frac{1}{\lambda}$. It can rewritten as

$$
\exp \left(-s^{\varepsilon}(\lambda) / 2\right)\left(s^{\varepsilon}\right)^{\prime}(\lambda) \leq \widehat{C}\left(\left(s^{\varepsilon}\right)^{\prime}(\lambda) t_{s^{\varepsilon}(\lambda)}^{\varepsilon}\right)^{\frac{1}{2}}
$$

and it is used in the remaining term in the right hand side of (5.18). It yields

$$
\begin{aligned}
& \left\|\left(U_{s^{\varepsilon}(\lambda), p, \operatorname{sign}(\lambda)}^{\varepsilon}, U\right) \exp \left(-s^{\varepsilon}(\lambda) / 2\right)\left(s^{\varepsilon}\right)^{\prime}(\lambda)\right\|_{L_{\lambda}^{2}(\mathbb{R})}^{2} \\
& \leq C\left(\left|\left(U_{s^{\varepsilon}, p,+}^{\varepsilon}, U\right)\right|_{+}^{\varepsilon}\right)^{2}+C\left(\left|\left(U_{s^{\varepsilon},-p,-}^{\varepsilon}, U\right)\right|_{-}^{\varepsilon}\right)^{2} .
\end{aligned}
$$

Plugging (5.20-5.22) in (5.18) yields the first inequality of the claim.

The second inequality of the claim is proved by the same method. It does not change (5.20), only (5.21)-(5.22) which are written for $z=c$. The proof is ended.

5.3.3. Bounds for large $|p|$. Due to the power $|k|^{-\frac{1}{2}}|p|^{-\frac{3}{2}}$, the following bounds are good ones for large values of $|p|$.

Lemma 5.12. One has the bounds

$$
\begin{aligned}
& \left|\begin{array}{l}
\widehat{F}_{p}^{\varepsilon} \\
\cdot, k, z
\end{array}\right|_{z}^{\varepsilon} \leq C \varepsilon\left(\frac{1}{|k|^{\frac{1}{2}}|p|^{\frac{3}{2}}}+\frac{1}{|k p|}\right)\left(\left|\left(U_{s^{\varepsilon}, p,+}^{\varepsilon}, U\right)\right|_{+}^{\varepsilon}+\left|\left(U_{s^{\varepsilon},-p,-}^{\varepsilon}, U\right)\right|_{-}^{\varepsilon}\right) \\
& \text { and }\left.\left.\right|_{\widehat{G}_{p, k, z}^{\varepsilon}} ^{\varepsilon}\right|_{z} ^{\varepsilon} \leq C \varepsilon\left(\frac{1}{|k|^{\frac{1}{2}}|p|^{\frac{3}{2}}}+\frac{1}{|k p|}\right)\left(\left|\left(U_{s_{c}^{\varepsilon}, p, c}^{\varepsilon}, U\right)\right|_{c}^{\varepsilon}+\left|\left(U_{s_{c}^{\varepsilon},-p, c}^{\varepsilon}, U\right)\right|_{c}^{\varepsilon}\right) .
\end{aligned}
$$

Proof. It is sufficient to prove the first estimate. The proof starts by showing a particular decomposition of (5.16) (still with $e=s_{z}^{\varepsilon}(|\lambda|)$ and $s=s^{\varepsilon}(|\mu|)$ )

$$
\begin{aligned}
\widehat{F}_{e, k, z}^{\varepsilon}= & \frac{k}{p} \int_{x \in I} \lambda f(\lambda, x)\left(\frac{1}{\pi} P . V . \int_{\mu \in \mathbb{R}} \frac{g(\mu, x)}{2 \mathbf{i} \pi k \lambda-2 \mathbf{i} p \mu} d \mu\right) d x \\
& -\frac{1}{2 \mathbf{i} \pi p} \int_{x \in I} f(\lambda, x)\left(\frac{1}{\pi} \int_{\mu \in \mathbb{R}} g(\mu, x) d \mu\right) d x
\end{aligned}
$$


from which the claim is deduced by methods similar to the previous lemma. The only technical difference is that $f$ is replaced by $\lambda f$ and $\mu g$ replaced by $g$. So (5.19) is replaced by $\int_{\mathbb{R}^{+}} e^{-s_{z}^{\varepsilon}(\lambda)} \lambda\left(s_{z}^{\varepsilon}\right)^{\prime}(\lambda) d \lambda \leq \overline{\bar{C}}$ and (5.21) is replaced by $e^{-s^{\varepsilon}(\lambda) / 2} \frac{1}{\lambda}\left(s^{\varepsilon}\right)^{\prime}(\lambda) \leq \widehat{C}\left(\left(s^{\varepsilon}\right)^{\prime}(\lambda) t_{s^{\varepsilon}(\lambda)}^{\varepsilon}\right)^{\frac{1}{2}}$. The proof is ended.

5.3.4. Proof of the completness Theorem 5.9. The first estimate establishes that $V_{\cdot, k, z}^{\varepsilon}-U_{\cdot, k, z}^{\varepsilon}$ is small for small $\varepsilon$.

Proposition 5.13. There is $C>0$ such that $\left.\mid\left(V_{\cdot, k, z}^{\varepsilon}-U_{\cdot, k, z}^{\varepsilon}\right), U\right)\left.\right|_{z} ^{\varepsilon} \leq \varepsilon \frac{C}{|k|}|U|^{\varepsilon}$.

Proof. The proof is a combination of elementary steps with the use of the bound (5.9) in combination with the inequalities of the previous Sections 5.3.2 and 5.3.3.

- By (5.9) one has $\left|a_{e, k, z}^{\varepsilon}(x) e_{0}, U\right|_{z} \leq \varepsilon \frac{d_{\varphi}}{|k|}|U|^{\varepsilon}$ where $d_{\varphi}>0$ is independent of $z$ and $k \neq 0$.

- Cutting the sums for $0<|p| \leq|k|$ and for $|k|<|p|$, one gets

$$
\begin{gathered}
\sum_{p \neq 0}\left|\widehat{F}_{p, k, z}^{\varepsilon}\right|_{z}^{\varepsilon} \leq \sum_{0<|p| \leq|k|} \frac{C \varepsilon}{|k|^{\frac{3}{2}}|p|^{\frac{1}{2}}}\left(\left|\left(U_{s^{\varepsilon}, p,+}^{\varepsilon}, U\right)\right|_{+}^{\varepsilon}+\left|\left(U_{s^{\varepsilon},-p,-}^{\varepsilon}, U\right)\right|_{-}^{\varepsilon}\right) \\
+\sum_{|k|<|p|} C \varepsilon\left(\frac{1}{|k|^{\frac{1}{2}}|p|^{\frac{3}{2}}}+\frac{1}{|k p|}\right)\left(\left|\left(U_{s^{\varepsilon}, p,+}^{\varepsilon}, U\right)\right|_{+}^{\varepsilon}+\left|\left(U_{s^{\varepsilon},-p,-}^{\varepsilon}, U\right)\right|_{-}^{\varepsilon}\right) \\
\leq \sum_{0<|p| \leq|k|} \frac{C \varepsilon}{|k||p|}\left(\left|\left(U_{s^{\varepsilon}, p,+}^{\varepsilon}, U\right)\right|_{+}^{\varepsilon}+\left|\left(U_{s^{\varepsilon},-p,-}^{\varepsilon}, U\right)\right|_{-}^{\varepsilon}\right) \\
+\sum_{|k|<|p|} C \varepsilon\left(\frac{1}{|k||p|}+\frac{1}{|k p|}\right)\left(\left|\left(U_{s^{\varepsilon}, p,+}^{\varepsilon}, U\right)\right|_{+}^{\varepsilon}+\left|\left(U_{s^{\varepsilon},-p,-}^{\varepsilon}, U\right)\right|_{-}^{\varepsilon}\right) \\
\leq \frac{C \varepsilon}{|k|} \sum_{p \neq 0}\left(\frac{1}{|p|}\left(\left|\left(U_{s^{\varepsilon}, p,+}^{\varepsilon}, U\right)\right|_{+}^{\varepsilon}+\left|\left(U_{s^{\varepsilon},-p,-}^{\varepsilon}, U\right)\right|_{-}^{\varepsilon}\right)\right) \\
\leq \frac{C \varepsilon}{|k|} \sqrt{\sum_{p \neq 0} \frac{1}{p^{2}}} \sqrt{\sum_{p \neq 0}\left(\left|\left(U_{s^{\varepsilon}, p,+}^{\varepsilon}, U\right)\right|_{+}^{\varepsilon}\right)^{2}} \leq \frac{C \varepsilon}{|k|}|U|^{\varepsilon} .
\end{gathered}
$$

- A similar inequality is proved for the other sum in (5.12) So the proof.

The second estimate yields that $V_{k}^{\varepsilon}-\exp (2 \mathbf{i} \pi k x) e_{0}$ is small for small $\varepsilon$.

Lemma 5.14. One has the inequality $\left|\left(V_{k}^{\varepsilon}-\exp (2 \mathbf{i} \pi k x) e_{0}, U\right)\right| \leq \frac{C \varepsilon}{|k|}|| U||$.

Proof. By definition (4.2) for $k \in \mathbb{Z}^{*}$, one has $V_{k}^{\varepsilon}(x)=\exp (2 \mathbf{i} \pi k x) e_{0}+$ $\alpha \varepsilon g(x) e_{1}$ where $-\exp \left(-\varphi_{0}(x) / 2\right) g(x)=\frac{1}{2 \mathbf{i} \pi k} \exp (2 \mathbf{i} \pi k x)$. End of proof.

The final proof of the completness Theorem is now an easy task. 
Proof of Theorem 5.9. The first inequality \|\|$U\left\|^{\varepsilon} \leq(1+C \varepsilon)\right\| U \|$ is proved as follows. Proposition 5.13 yields $(0<\varepsilon \leq 1)$

$$
\begin{gathered}
\sum_{z} \sum_{k \neq 0}\left(\left|\left(V_{\cdot, k, z}^{\varepsilon}, U\right)\right|_{z}^{\varepsilon}\right)^{2} \leq \sum_{z} \sum_{k \neq 0}\left(\left|\left(U_{\cdot, k, z}^{\varepsilon}, U\right)\right|_{z}^{\varepsilon}+\varepsilon \frac{C}{|p|}|U|^{\varepsilon}\right)^{2} \\
\leq(1+\varepsilon) \sum_{z} \sum_{k \neq 0}\left(\left|\left(U_{\cdot, k, z}^{\varepsilon}, U\right)\right|_{z}^{\varepsilon}\right)^{2}+\left(\varepsilon+\varepsilon^{2} C^{2} \sum_{k \neq 0} \frac{1}{|p|^{2}}\right)\left(|U|^{\varepsilon}\right)^{2} \\
\leq \sum_{z} \sum_{k \neq 0}\left(\left|\left(U_{\cdot, k, z}^{\varepsilon}, U\right)\right|_{z}^{\varepsilon}\right)^{2}+\widehat{C} \varepsilon\left(|U|^{\varepsilon}\right)^{2} .
\end{gathered}
$$

From Lemma 5.14, one gets similarly

$$
\begin{gathered}
\sum_{z} \sum_{k \neq 0}\left(\left|\left(V_{k}^{\varepsilon}, U\right)\right|_{z}^{\varepsilon}\right)^{2} \\
\leq(1+\varepsilon) \sum_{z} \sum_{k \neq 0}\left(\left|\left(\exp (2 \mathbf{i} \pi k x) e_{0}, U\right)\right|_{z}^{\varepsilon}\right)^{2}+C \varepsilon\|U\|^{2} \leq\left\|U \cdot e_{0}\right\|^{2}+\widetilde{C} \varepsilon\|U\|^{2} .
\end{gathered}
$$

By construction $\sum_{z}\left(\left|\left(V_{\cdot, 0, z}^{\varepsilon}, U\right)\right|_{z}^{\varepsilon}\right)^{2}=\sum_{z}\left(\left|\left(U_{\cdot, 0, z}^{\varepsilon}, U\right)\right|_{z}^{\varepsilon}\right)^{2}$. The addition is this equality to the two inequalities $(5.23-5.24)$ yields the first inequality of the Theorem.

The second inequality can be proved as an inverse inequality. Take $U \in X$ and note $\left(V_{e, k, z}^{\varepsilon}, U\right)=\alpha_{e, k, z}^{\varepsilon} \in \mathbb{C}$ and $\left(V_{k}^{\varepsilon}, U\right)=\alpha_{k}^{\varepsilon} \in \mathbb{C}$ for $k \neq 0$. The comparison estimates of Proposition 5.13 and Lemma 5.14 yield a way to determine $U$ directly from the $\alpha_{e, k, z}^{\varepsilon}$ and the $\alpha_{k}^{\varepsilon}$. Indeed using the representation of the identity, one obtains the linear equation $U=-T^{\varepsilon} U+b^{\varepsilon}$ where $T^{\varepsilon}$ is the operator defined by

$$
\begin{aligned}
T^{\varepsilon} U & =\sum_{k \neq 0}\left(U, V_{k}^{\varepsilon}-\exp (2 \mathbf{i} \pi k x) e_{0}\right) \exp (2 \mathbf{i} \pi k x) e_{0} \\
& +\sum_{z} \sum_{k \neq 0} \int_{I_{z}^{\varepsilon}}\left(U, V_{e, k, z}^{\varepsilon}-U_{e, k, z}^{\varepsilon}\right) t_{e}^{\varepsilon} d e
\end{aligned}
$$

and the right hand side is $b^{\varepsilon}=\sum_{k \neq 0} \alpha_{k}^{\varepsilon} \exp (2 \mathbf{i} \pi k x) e_{0}+\sum_{z} \sum_{k} \int_{I_{z}^{\varepsilon}} \alpha_{e, k, z}^{\varepsilon} t_{e}^{\varepsilon} d e$. The estimates of Proposition 5.13 and Lemma 5.14 and a Cauchy-Schwarz inequality yields the boundedness $\left\|T^{\varepsilon}\right\| \leq C \varepsilon$. Therefore the operator $I+T^{\varepsilon}$ is invertible with a continuous inverse under the non optimal sufficient condition that $C \varepsilon \leq 1 / 2$. That is $U=\left(I+T^{\varepsilon}\right)^{-1} b^{\varepsilon}$. Since $\left\|b^{\varepsilon}\right\|=\|U U\| \|^{\varepsilon}$, one gets $\|U\| \leq(1-C \varepsilon)^{-1}\||| U \mid\|^{\varepsilon}$. It yields the second inequality of the claim for $0<\varepsilon \leq \varepsilon_{*}$ and the proof is ended. 


\section{Proof of the main Theorem 1.7}

It is merely a consequence of a spectral representation with the $V_{e, k, z}^{\varepsilon}$. A last but necessary step is needed to make sure that the $V_{e, 0, z}^{\varepsilon}=U_{e, 0, z}^{\varepsilon}$ do not show up in the final representation formula (6.2) or (1.10). Indeed if the index $k=0$ shows up in the series, then the exponential term would be constant $e^{\lambda_{e, 0}^{\varepsilon} t}=e^{0 t}=1$ : in this case no phase mixing is possible.

Let us define

$$
X_{*}^{\varepsilon}=\left\{U \in X:\left(U, V_{f}^{\varepsilon}\right)=0 \forall f \in L_{0}^{2}(I),\left(U, V_{e, 0, z}^{\varepsilon}\right)=0 \forall z \text { and } e \in I_{z}^{\varepsilon}\right\} .
$$

The hermitian products $\left(U, V_{k}\right)=0$ mean that the Gauss law is satisfied, Proposition 4.3. In view of the equivalence of norms of Theorem 5.9, define the bounded operator $L^{\varepsilon}: X \rightarrow X$

$$
L^{\varepsilon} Z=\sum_{z} \sum_{k \neq 0} \int_{I_{z}^{\varepsilon}}\left(Z, U_{e, k, z}^{\varepsilon}\right) V_{e, k, z}^{\varepsilon} t_{e}^{\varepsilon} d e
$$

This operator is a kind of modified wave operator [55].

Lemma 6.1. $\operatorname{Im}\left(L^{\varepsilon}\right)$ is closed for all $0<\varepsilon<\varepsilon_{*}$.

Proof. We provide the proof for the completness of this work. The adjoint operator is $\left(L^{\varepsilon}\right)^{*} Z=\sum_{z} \sum_{k \neq 0} \int_{I_{z}^{\varepsilon}}\left(Z, V_{e, k, z}^{\varepsilon}\right) U_{e, k, z}^{\varepsilon} t_{e}^{\varepsilon} d e$ and we use the characterization of Theorem 2.19 in [12]. Take a sequence $Z_{n} \in X$ and assume $Y_{n}=\left(L^{\varepsilon}\right)^{*} Z_{n} \rightarrow Y \in X$ as $n \rightarrow \infty$. We need to show that there exists $Z \in X$ such that $Y=\left(L^{\varepsilon}\right)^{*} Z$. In view of the representation of the identity (3.23), it is sufficient to find $Z \in X \operatorname{such}$ that $\left(Z, V_{e, k, z}^{\varepsilon}\right):=\alpha_{e, k, z}^{\varepsilon}=$ $\left(Y, U_{e, k, z}^{\varepsilon}\right)$ for $k \neq 0$. Decide that $\left(Z, V_{e, 0, z}^{\varepsilon}\right):=\alpha_{e, 0, z}^{\varepsilon}=0$ for $k=0$ and that $\left(Z, \exp (2 \mathbf{i} \pi k z) \mathbf{e}_{0}\right):=\alpha_{k}^{\varepsilon}=0$. Then $U=\left(I+T^{\varepsilon}\right)^{-1} b^{\varepsilon}$ defined with (5.25) is the solution that is seek for. It ends the proof.

Proposition 6.2. $\operatorname{Im}\left(L^{\varepsilon}\right)=X_{*}^{\varepsilon}$ for $0<\varepsilon<\varepsilon_{*}$.

Proof. Since $\operatorname{Im}\left(L^{\varepsilon}\right)$ is closed, then [12] $\operatorname{Im}\left(L^{\varepsilon}\right)=\operatorname{Ker}\left(\left(L^{\varepsilon}\right)^{*}\right)^{\perp}$ where $\left(L^{\varepsilon}\right)^{*} Z=$ $\sum_{z} \sum_{k \neq 0}\left(Z, V_{e, k, z}^{\varepsilon}\right) U_{e, k, z}^{\varepsilon}$. The spectral representation (3.23) shows that $\operatorname{Ker}\left(\left(L^{\varepsilon}\right)^{*}\right)=\left\{Z \in X:\left(Z, V_{e, k, z}^{\varepsilon}\right)=0 \forall e, z\right.$ and $\left.k \neq 0\right\}$. Take $Z \in \operatorname{Ker}\left(\left(L^{\varepsilon}\right)^{*}\right)$ and define for technical reasons $\widehat{Z}=Z-V_{Z}^{\varepsilon} \cdot e_{0}$. By construction $\widehat{Z} \cdot e_{0}=Z \cdot e_{0}-$ $Z \cdot e_{0}=0$ and $\left(\widehat{Z}, V_{e, k, z}^{\varepsilon}\right)=-\left(V_{Z \cdot e_{0}}^{\varepsilon}, V_{e, k, z}^{\varepsilon}\right)=-\frac{1}{\lambda_{e, k}^{\varepsilon}}\left(V_{Z \cdot e_{0}}^{\varepsilon}, \mathbf{i} H_{0}^{\varepsilon} V_{e, k, z}^{\varepsilon}\right)=$ $\frac{1}{\lambda_{e, k}^{\varepsilon}}\left(\mathbf{i} H_{0}^{\varepsilon} V_{Z \cdot e_{0}}^{\varepsilon}, V_{e, k, z}^{\varepsilon}\right)=0$ for $k \neq 0$. By Proposition $\left.5.13 \mid\left(\widehat{Z}, U_{\cdot, k, z}^{\varepsilon}\right)\right)\left.\right|_{z} ^{\varepsilon} \leq$ $\varepsilon \frac{c_{\varphi}}{|k|}|\widehat{Z}|^{\varepsilon}$. Since $\widehat{Z} \cdot e_{0}=0$, one obtains by summation with respect to $k$ and another Cauchy-Schwarz inequality $|\widehat{Z}|^{\varepsilon} \leq C \varepsilon|\widehat{Z}|^{\varepsilon}$. So $Z \in \operatorname{Ker}\left(\left(L^{\varepsilon}\right)^{*}\right)$ is equivalent to $|\widehat{Z}|^{\varepsilon}=0$ (for small enough $\varepsilon$ ). In view of the definition of the semi-norm one gets $Z \in \operatorname{Ker}\left(\left(L^{\varepsilon}\right)^{*}\right) \Longleftrightarrow \widehat{Z}=\sum_{z} \int_{I_{z}^{\varepsilon}} \alpha_{z}(e) U_{e, 0, z}^{\varepsilon} t_{e}^{\varepsilon} d e$ which equivalent to $Z=V_{f}^{\varepsilon}+\sum_{z} \int_{I_{z}^{\varepsilon}} \alpha_{z}(e) V_{e, 0, z}^{\varepsilon} t_{e}^{\varepsilon} d e$ where $f=Z \cdot e_{0}$. One has 
$U \in \operatorname{Ker}\left(\left(L^{\varepsilon}\right)^{*}\right)^{\perp} \Longleftrightarrow(U, Z)=0$ for all $Z \in \operatorname{Ker}\left(\left(L^{\varepsilon}\right)^{*}\right) \Longleftrightarrow\left(U, V_{f}^{\varepsilon}\right)=0$ for all $f \in L_{0}^{2}(I)$ and $\left(U, V_{e, 0, z}^{\varepsilon}\right)=0$ for all $z$ and $e \in I_{z}^{\varepsilon} \Longleftrightarrow U \in X_{*}^{\varepsilon}$. This is the claim.

Let us consider now $U_{0} \in X_{*}^{\varepsilon}$. Proposition 6.2 yields the existence of $Z_{0} \in X$ such that $L^{\varepsilon} Z_{0}=U_{0}$.

Proposition 6.3. Assume $0<\varepsilon<\varepsilon_{*}$. The solution of $U^{\prime}(t)=\mathbf{i} H^{\varepsilon} U(t)$ with the initial data $U(0)=U_{0} \in X_{*}^{\varepsilon}$ is $U(t)=\sum_{z} \sum_{k \neq 0} \int_{I_{z}^{\varepsilon}}\left(Z_{0}, U_{e, k, z}^{\varepsilon}\right) e^{t \lambda_{e, k}^{\varepsilon}} V_{e, k, z}^{\varepsilon} t_{e}^{\varepsilon} d e$ (which is convergent in $X$ ).

Proof. The initial condition is satisfied (6.1). One has

$$
\begin{gathered}
U^{\prime}(t)=\sum_{z} \sum_{k \neq 0} \int_{I_{z}^{\varepsilon}}\left(Z_{0}, U_{e, k, z}^{\varepsilon}\right) e^{t \lambda_{e, k}^{\varepsilon} \lambda_{e, k}^{\varepsilon} V_{e, 0, z}^{\varepsilon} t_{e}^{\varepsilon} d e} \\
=\sum_{z} \sum_{k \neq 0} \int_{I_{z}^{\varepsilon}}\left(Z_{0}, U_{e, k, z}^{\varepsilon}\right) e^{t \lambda_{e, k}^{\varepsilon}}\left(\mathbf{i} H^{\varepsilon} V_{e, 0, z}^{\varepsilon}\right) t_{e}^{\varepsilon} d e=\mathbf{i} H^{\varepsilon} U(t) .
\end{gathered}
$$

The relation $\mathbf{i} H^{\varepsilon} V_{e, 0, z}^{\varepsilon}=\lambda_{e, k}^{\varepsilon} V_{e, 0, z}^{\varepsilon}$ is because it is equivalent to (4.5). A separate proof in the weak sense is in Lemma D.1. This relation is fully justified using arguments which are standard in scattering theory [33, 38, 29, $55,25,49]$.

Theorem 6.4 (Linear Landau damping for non constant Boltzmanian states.). Assume $0<\varepsilon<\varepsilon_{*}$. Let $V \in X$. Then one has $\lim _{t \rightarrow \infty}(U(t), V)=0$. Moreover the electric field $E=U \cdot e_{0}$ is such that $\lim _{t \rightarrow \infty}\|E(t)\|_{L^{\infty}(I)}=0$.

Proof. One has

$$
(U(t), V)=\sum_{z} \sum_{k \neq 0} \int_{I_{z}^{\varepsilon}}\left(U_{0}, U_{e, k, z}^{\varepsilon}\right) e^{t \lambda_{e, k}}\left(V_{e, 0, z}^{\varepsilon}, V\right) t_{e}^{\varepsilon} d e
$$

where the sum is convergent in view of theorem 5.9. The terms $e^{t \lambda_{e, k}^{\varepsilon}}$ are complex numbers of modulus one, which oscillate more and more as $t \rightarrow \infty$ since $\lambda_{e, k}^{\varepsilon}=-2 \mathbf{i} \pi k \lambda \in \mathbf{i} \mathbb{R}^{*}$. One can make the change of variable $e \mapsto \lambda$ in the integrals, it reveals one more time the importance of the monotony of the time of travel with respect to the label: using the Riemann-Lebesgue lemma, one obtains the first claim.

Indeed the Gauss law yields control of the electric field $E(t)$ in $H^{1}(I)$, that is $\left\|\partial_{x} E(t)\right\|_{L^{2}(I)} \leq C<\infty$. By compactness of the unit ball in $L_{0}^{2}(I) \cap$ $H^{1}(I)$ into $L_{0}^{2}(I)$, one gets that there exists a limit $E_{\infty}$ such that $E(t)$ tends to $E_{\infty}$ as $t \rightarrow \infty$. The limit is by subsequences. Take $V(x)=E_{\infty}(x) e_{0}$ in the weak limit. One gets that at the limit that $E_{\infty}=0$ in $L_{0}^{2}(I)$. Since the limit is the same for all subsequences, one gets $\lim _{t \rightarrow \infty}\|E(t)\|_{L^{2}(I)}=0$. Interpolation between $L^{2}(I)$ and $H^{1}(I)$ yields convergence in the $L^{\infty}(I)$ norm and the proof is ended. 
Remark 6.5. Estimates of the regularity with respect to $e$ of all terms in the representation formula (6.2) yield estimates of the rates on the decay of the electric field. In theory, it is possible to justify the regularity and summability of all terms, and $p$ integration by parts yield terms like $O\left(t^{-p}\right)$ in usual norms. The technical issue is near the separatrix.

\section{Appendix A. Monotony of the the time of travel}

\section{A.1. First branch: $e \in\left(-\varphi_{0}^{-}, \infty\right)$}

We distinguish the behavior for large $e>>1$ in Lemma A.1, near the lower bound $e=-\varphi_{0}^{-}+s$ for small $s>0$ in Lemma A.2 and the monotony in between in Lemma A.3. It allows to define properly the inverse function in Proposition 3.8.

Lemma A.1 (Evident). One has $\widehat{t}_{e}=\frac{1}{\sqrt{2 e}}+O\left(\frac{1}{e^{\frac{3}{2}}}\right)$ for large $e>>1$.

Lemma A.2. There exists a constant $C \in \mathbb{R}$ such that

$$
\widehat{t}_{-\varphi_{0}^{-}+\bar{s}}=-\frac{1}{\sqrt{\varphi_{0}^{\prime \prime}(0)}} \log \bar{s}+C+O(\bar{s}), \quad \bar{s}>0 .
$$

Proof. One has $\widehat{t}_{-\varphi_{0}^{-}+\bar{s}}=\int_{0}^{1} \frac{d z}{\sqrt{2\left(\bar{s}+\varphi_{0}(z)-\varphi_{0}^{-}\right)}}$. Consider $A=\int_{0}^{\frac{1}{2}} \frac{d z}{\sqrt{2\left(\bar{s}+\varphi_{0}(z)-\varphi_{0}^{-}\right)}}$ which is decomposed as $A=\underbrace{\int_{0}^{\frac{1}{2}} \frac{d z}{\sqrt{2\left(\bar{s}+\frac{1}{2} \varphi_{0}^{\prime \prime}(0) z^{2}\right)}}}_{=A_{1}}$ $+\underbrace{\int_{0}^{\frac{1}{2}}\left(\frac{1}{\sqrt{2\left(\bar{s}+\varphi_{0}(z)-\varphi_{0}^{-}\right)}}-\frac{1}{\sqrt{2\left(\bar{s}+\frac{1}{2} \varphi_{0}^{\prime \prime}(0) z^{2}\right.}}\right)}_{=A_{2}} d z$. Make the change of variable $z=\sqrt{\frac{2 \bar{s}}{\varphi_{0}^{\prime \prime}(0)}} y$ and denote $a_{\bar{s}}=1 /\left(2 \sqrt{2 \bar{s} / \varphi_{0}^{\prime \prime}(0)}\right)$, so $A_{1}=\frac{1}{\sqrt{\varphi_{0}^{\prime \prime}(0)}} \int_{0}^{a_{\bar{s}}} \frac{d y}{\sqrt{1+y^{2}}}=$ $\frac{1}{\sqrt{\varphi_{0}^{\prime \prime}(0)}} \log \left(a_{\bar{s}}+\sqrt{1+a_{\bar{s}}^{2}}\right)$. So $A_{1}=-\frac{1}{2 \sqrt{\varphi_{0}^{\prime \prime}(0)}} \log \bar{s}+d_{\varphi_{0}}+O(\bar{s})$ for some constant $d_{\varphi_{0}}$. So $A=-\frac{1}{2 \sqrt{\varphi_{0}^{\prime \prime}(0)}} \log \bar{s}+e_{\varphi_{0}}+O(\bar{s})$ for some constant $e_{\varphi_{0}}$. The integral $B=\int_{\frac{1}{2}}^{1} \frac{d z}{\sqrt{2\left(\bar{s}+\varphi_{0}(z)-\varphi_{0}^{-}\right)}}$has the same asymptotic behavior. It yields the claim after summation of $A$ and $B$.

Lemma A.3 (Evident). In interval $\left(-\varphi_{0}^{-}, \infty\right), e \mapsto \widehat{t}_{e}$ is monotone decreasing from $+\infty$ to 0 .

Proof of Proposition 3.8. The first point is evident. Second point: let us shift for convenience the functions $\bar{s}(\lambda)=s(\lambda)+\varphi_{0}^{-}$and $\psi(t)=\varphi(t)-\varphi_{0}^{-}$. The expansion of $s(\lambda)$ is immediate from Lemma A.1. By successive derivations of the first equation of (3.14) one obtains $-\frac{\sqrt{2}}{\lambda^{2}}=-\frac{1}{2}\left(\int_{0}^{1} \frac{d t}{(\bar{s}(\lambda)+\psi(t))^{\frac{3}{2}}}\right) \bar{s}^{\prime}(\lambda)$, 
$\frac{2 \sqrt{2}}{\lambda^{3}}=-\frac{1}{2}\left(\int_{0}^{1} \frac{d t}{(\bar{s}(\lambda)+\psi(t))^{\frac{3}{2}}}\right) \bar{s}^{\prime \prime}(\lambda)+\frac{3}{4}\left(\int_{0}^{1} \frac{d t}{(\bar{s}(\lambda)+\psi(t))^{\frac{5}{2}}}\right) \bar{s}^{\prime}(\lambda)^{2}$ and

$-\frac{6 \sqrt{2}}{\lambda^{4}}=-\frac{1}{2}\left(\int_{0}^{1} \frac{d t}{(\bar{s}(\lambda)+\psi(t))^{\frac{3}{2}}}\right) \bar{s}^{\prime \prime \prime}(\lambda)$

$+\frac{9}{4}\left(\int_{0}^{1} \frac{d t}{(s(\lambda)+\psi(t))^{\frac{5}{2}}}\right) s^{\prime}(\lambda) \bar{s}^{\prime \prime}(\lambda)-\frac{15}{8}\left(\int_{0}^{1} \frac{d t}{(\bar{s}(\lambda)+\psi(t))^{\frac{7}{2}}}\right) \bar{s}^{\prime}(\lambda)^{3}$. For large $\lambda$, the estimates $\mathrm{s}$ on $\bar{s}^{\prime}=s^{\prime}, \bar{s}^{\prime \prime}=s^{\prime \prime}$ and $\bar{s}^{\prime \prime \prime}=s^{\prime \prime \prime}$ are obtained one after the other, and so on for increasing order of derivation. Third point: for small $0<\lambda$, the expansion $\bar{s}(\lambda)=\alpha_{\varphi_{0}} e^{-\frac{\sqrt{\varphi_{0}^{\prime \prime}(0)}}{2 \lambda}}(1+\sigma(\lambda))$ is obtained from (A.1). Moreover we note that there exists $\alpha>0$ and $\beta$ such that $\alpha t^{2} \leq \psi(t) \leq \beta t^{2}$ on the interval $t \in[0,1 / 2]$. Therefore for small $\tau>0$ and $p>1$

$\frac{\beta_{p}}{\tau^{(p-1) / 2}} \leq 2 \int_{0}^{\frac{1}{2}} \frac{d t}{\left(\tau+\beta t^{2}\right)^{\frac{p}{2}}} \leq \int_{0}^{1} \frac{d t}{(\tau+\psi(t))^{\frac{p}{2}}} \leq 2 \int_{0}^{\frac{1}{2}} \frac{d t}{\left(\tau+\alpha t^{2}\right)^{\frac{p}{2}}} \leq \frac{\alpha_{p}}{\tau^{(p-1) / 2}}$

for some constants $\alpha_{p}$ and $\beta_{p}>0$. It yields the first bound $\left|\bar{s}^{\prime}(\lambda)\right| \leq c_{1} \frac{\bar{s}(\lambda)}{\lambda^{2}}$ and it is sufficient to insert this expression in the other identities to obtain the results for the first derivatives.

\section{A.2. Second branch: $e \in\left(-\varphi_{0}^{+},-\varphi_{0}^{-}\right)$}

We note $e=-\varphi_{0}^{-}-s \Longleftrightarrow \bar{s}=-e-\varphi_{0}^{-}$for small $s>0$.

Lemma A.4. There exists $C \in \mathbb{R}$ such that $\widehat{t}_{-\varphi_{0}^{-}-\bar{s}}=-\frac{1}{\sqrt{\varphi_{0}^{\prime \prime}(0)}} \log \bar{s}+C+\nu(\bar{s})$ for $\varphi_{0}^{+}-\varphi_{0}^{-}>\bar{s}>0$, where $\lim _{\bar{s} \rightarrow 0^{+}} \nu(\bar{s})=0$.

Proof. The proof is similar to the one of Lemma A.2. in (3.14). One cuts the second integral (3.14) in two pieces and study the first piece $A=2 \int_{\widehat{a}_{e}}^{x_{0}} \frac{d z}{\sqrt{2(\psi(z)-\bar{s})}}$ where $\psi(z)=\varphi_{0}(z)-\varphi_{0}^{-}$and $\psi^{\prime \prime}(0)=\varphi_{0}^{\prime \prime}(0)$. One has

$$
A=2 \sqrt{\frac{2}{\varphi_{0}^{\prime \prime}(0)}} \underbrace{\int_{\widehat{a}_{e}}^{x_{0}} \frac{\frac{\psi^{\prime}(z)}{2 \sqrt{\psi(z)}}}{\sqrt{2(\psi(z)-\bar{s})}} d z}_{=A_{1}}+2 \sqrt{\frac{2}{\varphi_{0}^{\prime \prime}(0)}} \underbrace{\int_{\widehat{a}_{e}}^{x_{0}} \frac{\sqrt{\frac{\varphi_{0}^{\prime \prime}(0)}{2}}-\frac{\psi^{\prime}(z)}{2 \sqrt{\psi(z)}}}{\sqrt{2(\psi(z)-\bar{s})}} d z}_{=A_{2}} .
$$

Note that $\psi\left(\widehat{a}_{e}\right)=\varphi_{0}\left(\widehat{a}_{e}\right)-\varphi_{0}^{-}=-e-\varphi_{0}^{-}=\bar{s}$. A change of variable $\psi(z)=\bar{s} u^{2}$ (the differential is $\frac{\psi^{\prime}(z)}{2 \sqrt{\psi(z)}} d z=\sqrt{\bar{s}} d u$ ) in the first integral yields $A_{1}=\frac{1}{\sqrt{2}} \int_{1}^{c} \frac{d u}{\sqrt{u^{2}-1}}=\frac{1}{\sqrt{2}} \log \left(c+\sqrt{c^{2}-1}\right)$ with $c=\sqrt{\psi\left(x_{0}\right) / \bar{s}}$. So there exists a constant $k_{\varphi_{0}}$ such that $A_{1}=-\frac{1}{2 \sqrt{2}} \log \bar{s}+k_{\varphi_{0}}+o(\bar{s})$. Concerning $A_{2}$, we notice that $D(z):=\frac{\sqrt{\frac{\varphi_{0}^{\prime \prime}(0)}{2}}-\frac{\psi^{\prime}(z)}{2 \sqrt{\psi(z)}}}{\sqrt{\psi(z)}}=\frac{\sqrt{2 \psi^{\prime \prime}(0) \psi(z)}-\psi^{\prime}(z)}{2 \psi(z)}$. But $\psi(0)=$ $\psi^{\prime}(0)=0, \psi^{\prime \prime}(0)>0$ and $\psi$ is of classe $W^{3, \infty}$. Therefore $D \in L^{\infty}(I)$ and $A_{2}$ admits a limit as $s \rightarrow 0^{+}: A_{2}=\int_{\widehat{a}_{e}}^{x_{0}} D(z) \frac{\sqrt{\psi(z)}}{\sqrt{2(\psi(z)-\bar{s})}} d z=\widetilde{C}+o(\bar{s})$. One obtains the asymptotic expansion of $A=A_{1}+A_{2}=-\frac{1}{2 \sqrt{2}} \log \bar{s}+$ 
$\widehat{C}+o(\bar{s})$. Adding a similar contribution for the second part of the integral $B=2 \sqrt{\frac{\varphi_{0}^{\prime \prime}(0)}{2}} \int_{x_{0}}^{\widehat{b}_{e}} \frac{d z}{\sqrt{2(\psi(z)-\bar{s})}}$ ends the proof.

Lemma A.5. Under assumption 1.4, one has

$\widehat{t}_{-\varphi_{0}^{+}+\bar{e}}=\frac{\sqrt{2} \pi}{\sqrt{\psi_{0}^{\prime \prime}\left(x_{0}\right)}}+\frac{4 \sqrt{2} \pi\left(-\left(\psi^{\frac{1}{2}}\right)^{\prime \prime}\left(x_{0}\right)\right)}{\left(\psi^{\prime \prime}\right)\left(x_{0}\right)^{\frac{3}{2}}} \sqrt{\bar{e}}+o(\bar{e}), \quad$ where $\lim _{\bar{e} \rightarrow 0} o(\bar{e}) / \bar{e}=0$.

Proof. With the notations of Lemma 3.9, consider (3.15) and make a local expansion of $u g_{u}(\bar{e})$ for small $\bar{e}$. One has $\bar{e} u^{2}=\psi(z)=\frac{1}{2} \psi^{\prime \prime}\left(x_{0}\right)\left(z-x_{0}\right)^{2}+$ $O\left(z-x_{0}\right)^{3}$ and $\psi^{\prime}(z)=\psi^{\prime \prime}\left(x_{0}\right)\left(z-x_{0}\right)+O\left(z-x_{0}\right)^{2}$. So one has $\psi^{\prime}(z)=$ $\sqrt{2 \bar{e} \psi^{\prime \prime}\left(x_{0}\right)} u+O(\bar{e})$. Plugging in (3.16), one gets

$$
\lim _{\bar{e} \rightarrow 0^{+}} u g_{u}(\bar{e})=\lim _{\bar{e} \rightarrow 0^{+}} u \frac{\sqrt{\bar{e}}}{\psi^{\prime}(z)}=\frac{1}{\sqrt{2 \psi^{\prime \prime}\left(x_{0}\right)}} .
$$

One has (3.17), so one obtains also

$$
\sqrt{\bar{e}} \frac{d}{d \bar{e}} g_{u}(\bar{e})=\frac{2}{\left(2 \psi^{\prime \prime}\left(x_{0}\right)\right)^{\frac{3}{2}}}\left(-\psi^{\frac{1}{2}}\left(x_{0}\right)\right)^{\prime \prime}+O(\sqrt{\bar{e}}) .
$$

Use (A.3) and a primitive of $\left(\right.$ A.4), so $u g_{u}(\bar{e})=\frac{1}{\sqrt{2 \psi^{\prime \prime}\left(x_{0}\right)}}+\frac{4 u}{\left(2 \psi^{\prime \prime}\left(x_{0}\right)\right)^{\frac{3}{2}}}\left(-\psi^{\frac{1}{2}}\left(x_{0}\right)\right)^{\prime \prime} \sqrt{\bar{e}}+$ $O(\bar{e})$. Plug this Ansatz in (3.15) one gets

$H(\bar{e})=\int_{0}^{1} \frac{2}{\sqrt{1-u^{2}}}\left(\frac{1}{\sqrt{2 \psi^{\prime \prime}\left(x_{0}\right)}}+\frac{4 u}{\left(2 \psi^{\prime \prime}\left(x_{0}\right)\right)^{\frac{3}{2}}}\left(-\psi^{\frac{1}{2}}\left(x_{0}\right)\right)^{\prime \prime} \sqrt{\bar{e}}+O(\bar{e})\right) d u=\frac{\pi}{\sqrt{2 \psi^{\prime \prime}\left(x_{0}\right)}}+$ $\frac{8}{\left(2 \psi^{\prime \prime}\left(x_{0}\right)\right)^{\frac{3}{2}}}\left(-\psi^{\frac{1}{2}}\left(x_{0}\right)\right)^{\prime \prime} \sqrt{\bar{e}}+O(\bar{e})$. Adding the contribution which corresponds to $\int_{\widehat{a}_{e}}^{x_{0}} \ldots$ yields the claim.

Proof of Proposition 3.10. The first point is just a rephrasing of the previous results. The second point is the reciprocal expansion to the one of Lemma A.5. Indeed $\frac{1}{\lambda}-\frac{1}{\mathcal{B}}=\alpha \sqrt{\bar{e}}+\ldots$ yields $\bar{e}=\frac{(\lambda-\mathcal{B})^{2}}{\alpha^{2} \lambda^{2} \mathcal{B}^{2}}+\cdots=\frac{(\lambda-\mathcal{B})^{2}}{\alpha^{2} \mathcal{B}^{4}}+\ldots$ The coefficient in front of $(\lambda-\mathcal{B})^{2}$ in the Proposition is precisely $\frac{1}{\alpha^{2} \mathcal{B}^{4}}$. The estimate for the derivative can be obtain (as before) by differentiation $-\frac{1}{\lambda^{2}}=$ $s_{c}^{\prime}(\lambda) \frac{d}{d e}\left(2 \int_{\widehat{a}_{e}}^{\widehat{b}_{e}} \frac{d x}{\sqrt{2\left(e+\varphi_{0}(x)\right)}}\right)_{e=s_{c}(\lambda)}$. The derivative is $O\left(1 / \sqrt{s_{c}(\lambda)+\varphi_{0}^{+}}\right)$by means of (3.18)-(A.4). Therefore with $\lambda \approx \mathcal{B}$, one gets $s_{c}^{\prime}(\lambda)=O\left(\sqrt{s_{c}(\lambda)+\varphi_{0}^{+}}\right)=$ $O(\lambda-\mathcal{B})$. The proof of the last point is similar to Prop. 3.8.

\section{Appendix B. A technical result}

The technical result below is an essential step to characterize, in Proposition 5.6 , of the regularity of the $n_{a, b, p}^{\varepsilon}$ which are the bilinear forms in the region of trapped particles. The proof is elementary, however it is reasonable to think that it solves a fundamental issue. Indeed trapped particles, also called 
electrons hole, generate Abel type integrals [31][equations (5) and (6)] which need specific treatment to control their singularity.

We use the notations of Section 3.1.4. For $0<\lambda<\mathcal{B}$ and $\widehat{a}_{s_{c}(\lambda)} \leq$ $x \leq \widehat{b}_{s_{c}(\lambda)}$, let us define $\widehat{f}(\lambda)=\int_{\widehat{a}_{s_{c}(\lambda)}}^{\widehat{b}_{s_{c}(\lambda)}} \sin \left(2 \pi p \lambda y_{\lambda}(x)\right) a(x) d x$ and $y_{\lambda}(x)=$ $\int_{\widehat{a}_{s_{c}(\lambda)}}^{x} \frac{d t}{\sqrt{2\left(s_{c}(\lambda)+\varphi_{0}(t)\right)}}$. Our goal is to investigate the regularity of $\widehat{f}$, with the constraint that the regularity constant must be expressed in terms of the quadratic norm of $a$. The key issue is that it is not possible to have such a bound for the full derivative. Indeed, dropping non essential terms, one has the local expansion $y_{\lambda}(x)=\frac{\sqrt{2}}{\varphi_{0}^{\prime}\left(\widehat{a}_{s_{c}(\lambda)}\right)} \sqrt{x-\widehat{a}_{s_{c}(\lambda)}}+\ldots$ from which one gets $\frac{d}{d \lambda} y_{\lambda}(x)=\frac{C(\lambda)}{\sqrt{x-\widehat{a}_{s_{c}(\lambda)}}}+\ldots$ The problem is that $\frac{1}{\sqrt{x-\widehat{a}_{s_{c}(\lambda)}}} \notin L^{2}(I)$ so is not convenient to obtain good bounds with respect to $\|a\|_{L^{2}(I)}$. One is forced to characterize in a weaker space, typically a Hölder space. In the following we focus on 1/2-Hölder continuity with a weight which depends on $\lambda$.

Proposition B.1. There exists a constant $C>0$ independent of $p, \lambda, \mu \in[0, \mathcal{B}]$ and $a \in L^{2}(I)$ such that

$$
|\widehat{f}(\mu)-\widehat{f}(\lambda)| \leq C|p|\|a\|_{L^{2}(I)} \sqrt{\frac{|\lambda-\mu|}{\min \left(\varphi_{0}^{\prime}\left(\widehat{a}_{s_{c}(\lambda)}\right), \varphi_{0}^{\prime}\left(\widehat{b}_{s_{c}(\lambda)}\right)\right)}}, \quad \lambda<\mu .
$$

The proof is is a corollary of Proposition B.3 and Lemma B.4 below. The starting point is the following simple inequality. By definition (3.14), one has $\frac{1}{2 \lambda}=y_{\lambda}\left(\widehat{b}_{s_{c}(\lambda)}\right)$. For convenience we extend by continuity the fonction $y_{\lambda}$ in the entire interval $[0,1]$ setting $y_{\lambda}(x)=0$ for $0 \leq x \leq \widehat{a}_{s_{c}(\lambda)}$ and $y_{\lambda}(x)=$ $1 /(2 \lambda)$ for $\widehat{b}_{s_{c}(\lambda)} \leq x \leq 1$. Using the inequality $|\sin \alpha-\sin \beta| \leq|\alpha-\beta|$, one gets from the definition $|\widehat{f}(\mu)-\widehat{f}(\lambda)| \leq 2 \pi|p|\|a\|_{L^{2}(I)}\left\|\lambda y_{\lambda}-\mu y_{\mu}\right\|_{L^{\infty}(I)}$.

Lemma B.2. For $0<\lambda<\mu<\mathcal{B}$, one has

$\left\|\lambda y_{\lambda}-\mu y_{\mu}\right\|_{L^{\infty}(I)}=\lambda \max \left(y_{\lambda}\left(\widehat{a}_{s_{c}(\mu)}\right), \frac{1}{2}-y_{\lambda}\left(\widehat{b}_{s_{c}(\mu)}\right)\right)$.

Proof. Split the interval $\left.[0,1]=\left[0, \widehat{a}_{s_{c}(\lambda)}\right] \cup\left[\widehat{a}_{s_{c}(\lambda)}, \widehat{a}_{s_{c}(\mu)}\right] \cup \mid \widehat{a}_{s_{c}(\mu)}, \widehat{b}_{s_{c}(\mu)}\right] \cup$ $\left[\widehat{b}_{s_{c}(\mu)}, \widehat{b}_{s_{c}(\lambda)}\right] \cup\left[\widehat{b}_{s_{c}(\lambda)}, 1\right]$ for $0<\lambda<\mu<1$. By construction, the derivative of $g=\lambda y_{\lambda}-\mu y_{\mu}$ is zero in $\left(0, \widehat{a}_{s_{c}(\lambda)}\right)$, positive in $\left(\widehat{a}_{s_{c}(\lambda)}, \widehat{a}_{s_{c}(\mu)}\right)$, positive in $\left(\widehat{b}_{s_{c}(\mu)}, \widehat{b}_{s_{c}(\lambda)}\right)$ and zero in $\left(\widehat{b}_{s_{c}(\lambda)}, 1\right)$. In the interval $\left(\widehat{a}_{s_{c}(\mu)}, \widehat{b}_{s_{c}(\mu)}\right)$ one gets that $g^{\prime}(x)=\frac{\lambda}{\sqrt{2\left(s_{c}(\lambda)+\varphi_{0}(x)\right)}}-\frac{\mu}{\sqrt{2\left(s_{c}(\mu)+\varphi_{0}(x)\right)}}$. Proposition 3.10 yields that $s_{c}$ is decreasing function. Since $\lambda<\mu$, then $s_{c}(\lambda)>s_{c}(\mu)$. So $g^{\prime}(x)<0$ in this central interval. Therefore $\|g\|_{L^{\infty}(I)}=\max \left(g\left(\widehat{a}_{s_{c}(\mu)}\right),-g\left(\widehat{b}_{s_{c}(\mu)}\right)\right)$. So the claim. 
Proposition B.3. There exists a constant $C>0$ independent of $p, \lambda, \mu \in[0, \mathcal{B}]$ and $a \in L^{2}(I)$ such that for $\lambda<\mu$

$$
|\widehat{f}(\mu)-\widehat{f}(\lambda)| \leq C|p|\|a\|_{L^{2}(I)} \max \left(\sqrt{\frac{\left|\widehat{a}_{s_{c}(\lambda)}-\widehat{a}_{s_{c}(\mu)}\right|}{\varphi_{0}^{\prime}\left(\widehat{a}_{s_{c}(\lambda)}\right)}}, \sqrt{\frac{\left|\widehat{b}_{s_{c}(\lambda)}-\widehat{b}_{s_{c}(\mu)}\right|}{\varphi_{0}^{\prime}\left(\widehat{b}_{s_{c}(\lambda)}\right)}}\right) .
$$

Proof. One studies $y_{\lambda}\left(\widehat{a}_{s_{c}(\mu)}\right)$. By symmetry it will be sufficient to get similar estimates for the other term $\frac{1}{2}-y_{\lambda}\left(\widehat{b}_{s_{c}(\mu)}\right)$. A first estimate is $\lambda y_{\lambda}\left(\widehat{a}_{s_{c}(\mu)}\right) \leq \frac{1}{2}$. Next $x \in\left[\widehat{a}_{s_{c}(\lambda)}, x_{0}\right]$, one can rewrite $y_{\lambda}(x)=\int_{\widehat{a}_{s_{c}(\lambda)}}^{x} \frac{d t}{\sqrt{2\left(\varphi_{0}(t)-\varphi_{0}\left(\widehat{a}_{s_{c}(\lambda)}\right)\right)}}$. Let $\widehat{a}_{s_{c}(\lambda)} \leq d \leq t \leq \widehat{a}_{s_{c}(\mu)}$, one has the expansion $\varphi_{0}(t)-\varphi_{0}\left(\widehat{a}_{s_{c}(\lambda)}\right)=$ $\varphi_{0}^{\prime}(d)\left(t-\widehat{a}_{s_{c}(\lambda)}\right)$. So one has for $\widehat{a}_{s_{c}(\lambda)} \leq t \leq \widehat{a}_{s_{c}(\mu)}$

$$
\varphi_{0}(t) \geq\left(\varphi_{0}^{\prime}\left(\widehat{a}_{s_{c}(\lambda)}\right)-C\left(\widehat{a}_{s_{c}(\mu)}-\widehat{a}_{s_{c}(\lambda)}\right)\right)\left(t-\widehat{a}_{s_{c}(\lambda)}\right)
$$

where we used the boundedness of the second derivative to lower bound $\varphi_{0}^{\prime}(d) \geq \varphi_{0}^{\prime}\left(\widehat{a}_{s_{c}(\lambda)}\right)-C\left(\widehat{a}_{s_{c}(\mu)}-\widehat{a}_{s_{c}(\lambda)}\right)$ with $C>0$. Assuming that $\mu$ is sufficiently close to $\lambda$, one gets another estimate

$y_{\lambda}(x) \leq \frac{1}{\sqrt{\varphi_{0}^{\prime}\left(a_{s_{c}(\lambda)}\right)-C\left(\widehat{a}_{s_{c}(\lambda)}-\widehat{a}_{s_{c}(\mu)}\right)}} \int_{\widehat{a}_{s_{c}(\lambda)}}^{x} \frac{d t}{\sqrt{2\left(t-\widehat{a}_{s_{C}(\lambda)}\right)}}=\frac{\sqrt{2\left(t-\widehat{a}_{s_{c}(\lambda)}\right)}}{\sqrt{\varphi_{0}^{\prime}\left(\widehat{a}_{s_{c}(\lambda)}\right)-C\left(\widehat{a}_{s_{c}(\lambda)}-\widehat{a}_{s_{c}(\mu)}\right)}}$.

Combine the two estimates and use the notation $g(u, v)=\min \left(1, \sqrt{\frac{2 u}{v-C u}}\right)$ : so $y_{\lambda}\left(\widehat{a}_{s_{c}(\mu)}\right) \leq g\left(\widehat{a}_{s_{c}(\mu)}-\widehat{a}_{s_{c}(\lambda)}, \varphi_{0}^{\prime}\left(\widehat{a}_{s_{c}(\lambda)}\right)\right)$. The function $g$ is homogenous of degree 0 and $g(w, 1) \leq C \sqrt{w}$ for some constant $C$. It yields $\lambda y_{\lambda}\left(\widehat{a}_{s_{c}(\mu)}\right) \leq$ $C \sqrt{\frac{\widehat{a}_{s_{c}(\mu)}-\widehat{a}_{s_{c}(\lambda)}}{\varphi_{0}^{\prime}\left(\widehat{a}_{s_{c}(\lambda)}\right)}}$. A similar bound bound for the other term ends the proof of the claim of Proposition B.3.

Lemma B.4. The functions $\lambda \mapsto \widehat{a}_{s_{c}(\lambda)}$ and $\lambda \mapsto \widehat{b}_{s_{c}(\lambda)}$ are uniformly differentiable for $\lambda \in[0, \mathcal{B}]$.

Proof. The proof is provided for the first function. One has $s_{c}(\lambda)+\varphi_{0}\left(\widehat{a}_{s_{c}(\lambda)}\right)=$ 0 so $\frac{d}{d \lambda} \widehat{a}_{s_{c}(\lambda)}=-\frac{s_{c}^{\prime}(\lambda)}{\varphi_{0}^{\prime}\left(\widehat{a}_{s_{c}(\lambda)}\right)}$ for $\lambda \in(0, \mathcal{B})$ where $s_{c}(\lambda) \in C^{1}[0, \mathcal{B}]$. Assumption 1.4 yields that $\varphi_{0}^{\prime}(\lambda)>0$ for $0<\lambda<\mathcal{B}$. The issue is the limit of the above expression for $0^{+}$and $\mathcal{B}^{-}$.

- For small $\lambda$, Proposition 3.8 yields $s_{c}^{\prime}(\lambda)=O\left(\exp \left(-\frac{\sqrt{\varphi_{0}^{\prime \prime}(0)}}{2 \lambda}\right) / \lambda^{2}\right)$. For small $x \approx 0$, one has that $\varphi_{0}^{\prime}(x)=O\left(\varphi_{0}(x)+\varphi_{0}^{-}\right)^{\frac{1}{2}}$ so

$$
\varphi_{0}^{\prime}\left(\widehat{a}_{s_{c}(\lambda)}\right)=O\left(\varphi_{0}\left(\widehat{a}_{s_{c}(\lambda)}\right)+\varphi_{0}^{-}\right)^{\frac{1}{2}}=O\left(\exp \left(-\frac{\sqrt{\varphi_{0}^{\prime \prime}(0)}}{2 \lambda}\right)\right)^{\frac{1}{2}}
$$

and $\frac{d}{d \lambda} \widehat{a}_{s_{c}(\lambda)}=O\left(\frac{\exp \left(-\frac{\sqrt{\varphi_{0}^{\prime \prime}(0)}}{4 \lambda}\right)}{\lambda^{2}}\right)$ for small $\lambda$. So $\lim _{\lambda \rightarrow 0^{+}} \frac{d}{d \lambda} \widehat{a}_{s_{c}(\lambda)}=0$.

- For $\lambda \approx \mathcal{B}$, Proposition 3.8 yields $s_{c}^{\prime}(\lambda)=O(\lambda-\mathcal{B})$. For $x \approx x_{0}$, one has 
that $\varphi_{0}^{\prime}(x)=O\left(\varphi_{0}(x)+\varphi_{0}^{+}\right)^{\frac{1}{2}}$ so

$$
\varphi_{0}^{\prime}\left(\widehat{a}_{s_{c}(\lambda)}\right)=O\left(\varphi_{0}\left(\widehat{a}_{s_{c}(\lambda)}\right)+\varphi_{0}^{+}\right)^{\frac{1}{2}}=O(\lambda-\mathcal{B}) .
$$

Therefore one has a limit $\lim _{\lambda \rightarrow \mathcal{B}^{-}} \frac{d}{d \lambda} \widehat{a}_{s_{c}(\lambda)}<\infty$.

- Since $\frac{d}{d \lambda} \widehat{a}_{s_{c}(\lambda)} \in C^{0}(0, \mathcal{B})$, the proof is ended.

\section{Appendix C. A useful inequality}

For $p, q \in \mathbb{R}^{*}$, one has the estimate $\left\|\int_{x \in I} f(\lambda, x)\left(\frac{1}{\pi} P . V \cdot \int_{\mu \in \mathbb{R}} \frac{g(\mu, x)}{p \lambda-q \mu} d \mu\right) d x\right\|_{L_{\lambda}^{2}}^{2}$ $\leq \frac{1}{|p q|} \int_{x \in I}\|f\|_{L_{\lambda}^{2}}^{2} d x \times \int_{x \in I}\|g\|_{L_{\lambda}^{2}}^{2} d x$ where $L_{\lambda}^{2}=L_{\lambda}^{2}(\mathbb{R})$ is the quadratic norm is with respect to the variable $\lambda \in \mathbb{R}$. The proof which uses the fact that the Hilbert transform is an isometry in $L^{2}$ is left to the reader.

\section{Appendix D. Verification that $V_{e, k, z}^{\varepsilon}$ is a generalized eigenvector}

We provide a separate algebraic proof that $V_{e, k, z}^{\varepsilon}$ is a generalized eigenvector of $\mathbf{i} H^{\varepsilon}$. The proof is in the weak sense, assuming convergence of the sums and integrals (refer to Remark 5.8). It yields a direct verification of the conditions (4.5).

Lemma D.1. One has $\left(V_{e, k, z}^{\varepsilon}, A W^{\prime}\right)+\left(V_{e, k, z}^{\varepsilon}, \varepsilon^{2} E_{0} B W\right)+\left(V_{e, k, z}^{\varepsilon}, D W\right)=$ $\lambda_{e, k}^{\varepsilon}\left(V_{e, k, z}^{\varepsilon}, W\right)$ for all $W \in X_{0}$.

Let us start from (5.11). One has for all $W \in X_{0}$

$$
\begin{aligned}
& \left(V_{e, k, z}^{\varepsilon}, A W^{\prime}\right)=\left(U_{e, k, z}^{\varepsilon}, A W^{\prime}\right)+\left(a_{e, k, z}^{\varepsilon}(x) e_{0}, A W^{\prime}\right) \\
& +\varepsilon \sum_{z^{\prime}} \sum_{p \neq 0} P . V . \int_{s \in I_{z^{\prime}}^{\varepsilon}} \frac{\left(\alpha a_{e, k, z}^{\varepsilon} e_{2}, \exp \left(\varepsilon^{2} \varphi_{0} / 2\right) U_{s, p, z^{\prime}}^{\varepsilon}\right)}{\lambda_{s, p}^{\varepsilon}-\lambda_{e, k}^{\varepsilon}}\left(U_{s, p, z^{\prime}}^{\varepsilon}, A W^{\prime}\right) t_{s}^{\varepsilon} d s
\end{aligned}
$$

and

$$
\begin{aligned}
& \left(V_{e, k, z}^{\varepsilon}, \varepsilon^{2} E_{0} B W\right)=\left(U_{e, k, z}^{\varepsilon}, \varepsilon^{2} E_{0} B W\right)+\left(a_{e, k, z}^{\varepsilon}(x) e_{0}, \varepsilon^{2} E_{0} B W\right) \\
& +\varepsilon \sum_{z^{\prime}} \sum_{p \neq 0} P . V . \int_{s \in I_{z^{\prime}}^{\varepsilon}} \frac{\left(\alpha a_{e, k, z}^{\varepsilon} e_{2}, \exp \left(\varepsilon^{2} \varphi_{0} / 2\right) U_{s, p, z^{\prime}}^{\varepsilon}\right)}{\lambda_{s, p}^{\varepsilon}-\lambda_{e, k}^{\varepsilon}}\left(U_{s, p, z^{\prime}}^{\varepsilon}, \varepsilon^{2} E_{0} B W\right) t_{s}^{\varepsilon} d s .
\end{aligned}
$$


Summation of (D.1-D.3) and the use of (3.12) which is satisfied for all $e, k, z$ yields

$$
\begin{aligned}
& \left(V_{e, k, z}^{\varepsilon}, A W^{\prime}\right)+\left(V_{e, k, z}^{\varepsilon}, \varepsilon^{2} E_{0} B W\right) \\
& =\left(U_{e, k, z}^{\varepsilon}, A W^{\prime}\right)+\left(U_{e, k, z}^{\varepsilon}, \varepsilon^{2} E_{0} B W\right) \\
& +\left(a_{e, k, z}^{\varepsilon}(x) e_{0}, A W^{\prime}\right)+\left(a_{e, k, z}^{\varepsilon}(x) e_{0}, \varepsilon^{2} E_{0} B W\right) \\
& +\varepsilon \sum_{z^{\prime}} \sum_{p \neq 0} P . V . \int_{s \in I_{z^{\prime}}^{\varepsilon}} \frac{\left(\alpha a_{e, k, z}^{\varepsilon} e_{2}, \exp \left(\varepsilon^{2} \varphi_{0} / 2\right) U_{s, p, z^{\prime}}^{\varepsilon}\right)}{\lambda_{s, p}^{\varepsilon}-\lambda_{e, k}^{\varepsilon}} \lambda_{s, p}^{\varepsilon}\left(U_{s, p, z^{\prime}}^{\varepsilon}, W\right) t_{s}^{\varepsilon} d s .
\end{aligned}
$$

So

$$
\begin{aligned}
& \left(V_{e, k, z}^{\varepsilon}, A W^{\prime}\right)+\left(V_{e, k, z}^{\varepsilon}, \varepsilon^{2} E_{0} B W\right)-\lambda_{e, k}^{\varepsilon}\left(V_{e, k, z}^{\varepsilon}, W\right) \\
& =\left(U_{e, k, z}^{\varepsilon}, A W^{\prime}\right)+\left(U_{e, k, z}^{\varepsilon}, \varepsilon^{2} E_{0} B W\right)-\lambda_{e, k}^{\varepsilon}\left(U_{e, k, z}^{\varepsilon}, W\right) \\
& +\left(a_{e, k, z}^{\varepsilon}(x) e_{0}, A W^{\prime}\right)+\left(a_{e, k, z}^{\varepsilon}(x) e_{0}, \varepsilon^{2} E_{0} B W\right)-\lambda_{e, k}^{\varepsilon}\left(a_{e, k, z}^{\varepsilon}(x) e_{0}, W\right) \\
& +\varepsilon \sum_{z^{\prime}} \sum_{p \neq 0} \int_{s \in I_{z^{\prime}}^{\varepsilon}}\left(\alpha a_{e, k, z}^{\varepsilon} e_{2}, \exp \left(\varepsilon^{2} \varphi_{0} / 2\right) U_{s, p, z^{\prime}}^{\varepsilon}\right)\left(U_{s, p, z^{\prime}}^{\varepsilon}, W\right) t_{s}^{\varepsilon} d s \\
& =0-\lambda_{e, k}^{\varepsilon}\left(a_{e, k, z}^{\varepsilon} e_{0}, W\right)+\varepsilon\left(\exp \left(\varepsilon^{2} \varphi_{0} / 2\right) \alpha a_{e, k, z}^{\varepsilon} e_{2}, W\right) \text { (use the identity (3.23)). }
\end{aligned}
$$

Let $a \in L_{0}^{2}(I)$ and $c \in L^{2}(I)$ denote the first and third component of the infinite vector $W$. One recasts this as

$$
\begin{gathered}
\left(V_{e, k, z}^{\varepsilon}, A W^{\prime}\right)+\left(V_{e, k, z}^{\varepsilon}, \varepsilon^{2} E_{0} B W\right)-\lambda_{e, k}^{\varepsilon}\left(V_{e, k, z}^{\varepsilon}, W\right) \\
=-\lambda_{e, k}^{\varepsilon}\left(a_{e, k, z}^{\varepsilon}, a\right)+\varepsilon \alpha\left(\exp \left(\varepsilon^{2} \varphi_{0} / 2\right) a_{e, k, z}^{\varepsilon}, c\right) .
\end{gathered}
$$

On the other hand another use of (5.11) yields

$$
\begin{aligned}
& \left(V_{e, k, z}^{\varepsilon}, D W\right)=\left(U_{e, k, z}^{\varepsilon}, D W\right)+\left(a_{e, k, z}^{\varepsilon} e_{0}, D W\right) \\
& +\varepsilon \sum_{z^{\prime}} \sum_{p \neq 0} P . V . \int_{s \in I_{z^{\prime}}^{\varepsilon}} \frac{\left(\alpha a_{e, k, z}^{\varepsilon} e_{2}, \exp \left(\varepsilon^{2} \varphi_{0} / 2\right) U_{s, p, z^{\prime}}^{\varepsilon}\right)}{\lambda_{s, p}^{\varepsilon}-\lambda_{e, k}^{\varepsilon}}\left(U_{s, p, z^{\prime}}^{\varepsilon}, D W\right) t_{s}^{\varepsilon} d s .
\end{aligned}
$$

One can write $D W=\alpha \exp \left(\varphi_{0} / 2\right) a e_{2}-\alpha 1^{*}\left(\exp \left(\varphi_{0} / 2\right) c\right) e_{0}$. So

$$
\begin{aligned}
& \left(V_{e, k, z}^{\varepsilon}, D W\right)=\left(U_{e, k, z}^{\varepsilon} \cdot e_{2}, \alpha \exp \left(\varepsilon^{2} \varphi_{0} / 2\right) a\right)-\left(a_{e, k, z}^{\varepsilon} e_{0}, \alpha 1^{*}\left(\exp \left(\varepsilon^{2} \varphi_{0} / 2\right) c\right) e_{0}\right) \\
& +\varepsilon \alpha \sum_{z^{\prime}} \sum_{p \neq 0} P . V . \int_{s \in I_{z^{\prime}}^{\varepsilon}} \frac{\left(\alpha a_{e, k, z}^{\varepsilon} e_{2}, \exp \left(\varepsilon^{2} \varphi_{0} / 2\right) U_{s, p, z^{\prime}}^{\varepsilon}\right)}{\lambda_{s, p}^{\varepsilon}-\lambda_{e, k}^{\varepsilon}}\left(U_{s, p, z^{\prime}}^{\varepsilon} \cdot e_{2}, \exp \left(\varepsilon^{2} \varphi_{0} / 2\right) a\right) t_{s}^{\varepsilon} d s .
\end{aligned}
$$

Since $a_{e, k, z}^{\varepsilon} \in L_{0}^{2}(I)$ is the unique solution to (4.6)-(5.3)-(5.7), one obtains

$$
\begin{gathered}
\left(V_{e, k, z}^{\varepsilon}, D W\right)=\alpha\left(U_{e, k, z}^{\varepsilon} \cdot e_{2}, \exp \left(\varepsilon^{2} \varphi_{0} / 2\right) a\right)-\alpha\left(a_{e, k, z}^{\varepsilon}, \exp \left(\varepsilon^{2} \varphi_{0} / 2\right) c\right) \\
+\frac{1}{\varepsilon} \lambda_{e, k}^{\varepsilon}\left(a_{e, k, z}^{\varepsilon}, a\right)-\alpha\left(\exp \left(\varepsilon^{2} \varphi_{0} / 2\right) U_{e, k, z}^{\varepsilon} \cdot e_{2}, a\right) .
\end{gathered}
$$


One adds to (D.2). So $\left(V_{e, k, z}^{\varepsilon}, A W^{\prime}\right)+\left(V_{e, k, z}^{\varepsilon}, \varepsilon^{2} E_{0} B W\right)-\lambda_{e, k}^{\varepsilon}\left(V_{e, k, z}^{\varepsilon}, W\right)+$ $\varepsilon\left(V_{e, k, z}^{\varepsilon}, D W\right)=0$.

\section{References}

[1] M. Abramowitz and I.A. Stegun, Handbook of Mathematical Functions with Formulas, Graphs, and Mathematical Tables, Dover Publications, 1972.

[2] A. V. Antonov, Remarks on the problem of stability in stellar dynamics, Soviet. Astr., AJ. 4 (1961), 859-867.

[3] R. Bachelard, F Staniscia, T. Dauxois, G. De Ninno and S. Ruffo Stability of inhomogeneous states in mean-field models with an external potential, J. Stat. Mech., P03022, 2011.

[4] M. Badsi, M. Campos-Pinto and B. Després, A minimization formulation for a bi-kinetic-sheath, Kinetic and Related Models, AIMS, 9, 4, 2016.

[5] C. Bardos and P. Degond, Existence globale et comportement asymptotique de la solution de l'équation de Vlasov-Poisson, C.R. Acad. Sc. Paris, t. 297, 321-324, 1983.

[6] J. Barré and Y.Y. Yamaguchi, On algebraic damping close to inhomogeneous Vlasov equilibria in multi-dimensional spaces, 2013 J. Phys. A, 46225501.

[7] J. Barré, A. Olivetti and Y.Y. Yamaguchi, Dynamics of perturbations around inhomogeneous backgrounds in the HMF model, Journal of Statistical Mechanics: Theory and Experiment, Volume 2010, 2010.

[8] J. Barré, A. Olivetti and Y.Y. Yamaguchi, Landau damping and inhomogeneous reference states, Comptes Rendus Physique, Vol. 16, 8, 723-728, 2015.

[9] J. Bedrossian, N. Masmoudi and C. Mouhot, Landau damping: paraproducts and Gevrey regularity. Ann. PDE 2, 2016.

[10] J. Bedrossian, N. Masmoudi and C. Mouhot, Landau damping in finite regularity for unconfined systems with screened interactions, arxiv preprint https: //arxiv.org/abs/1604.05783.

[11] J. Ben-Artzi, On the spectrum of shear flows and uniform ergodic theorems, J. of Functional Analysis 267, 1, 299-322, 2014.

[12] H. Brezis, Functional Analysis, Sobolev Spaces and Partial Differential Equations, Springer, 2011.

[13] K.M. Case, Plasma oscillations, Annals of Physics, 349-364, 1959.

[14] K.M. Case, Scattering theory, orthogonal polynomials, and the transport equation. J. Math. Phys. 15 (1974), 974-983.

[15] K.M. Case, Orthogonal polynomials from the viewpoint of scattering theory. J. Math. Phys. 15 (1974), 2166-2174.

[16] M. Cassier, C. Hazard and P. Joly, Spectral theory for Maxwell's equations at the interface of a metamaterial, Part I: Generalized Fourier transform, https: //arxiv.org/abs/1610.03021, 2016.

[17] N. Crouseilles, M. Mehrenberger and Sonnendrücker, Conservative semiLagrangian schemes for Vlasov equations, Journal of Computational Physics 229, 1927-1953, 2010. 
[18] B. Després, Symmetrization of Vlasov-Poisson equations. SIAM J. Math. Anal. 46 (2014), no. 4, 2554-2580.

[19] P. Degond, Spectral theory of the linearized Vlasov-Poisson equation, Trans. of the AMS, 294, 1986, 435-453.

[20] R.J. Diperna and P.L. Lions, Global weak solutions of Vlasov-Maxwell systems, Comm. on Pure and App. Math., Vol. XLII, 729-757, 1989.

[21] Y. Elskens, Irreversible behaviors in Vlasov equation and many-body hamiltonian dynamics: Landau damping, chaos and granularity in the kinetic limit, pp. 89-108 in Th. Passot, C. Sulem \& P-L. Sulem (eds), Topics in kinetic theory, Fields institute communications series 46, AMS, 2005.

[22] E. Faou and F. Rousset, Landau Damping in Sobolev Spaces for the VlasovHMF Model, Archive for Rational Mechanics and Analysis 2016, Volume 219, Issue 2, pp 887-902. 2016.

[23] F. Filbet and E. Sonnendrücker, Comparison of Eulerian Vlasov solvers, Comput. Phys. Comm. 150, no. 3, 247-266, 2003.

[24] F. Filbet, E. Sonnendrücker and P. Bertrand, Conservative numerical schemes for the Vlasov equation, JCP, 172, 166-187, 2001.

[25] C. Gérard and F. Nier, Scattering theory for the perturbations of periodic Schrödinger operators, J. Math. Kyoto Univ. Vol. 38, 4 (1998), 595-634.

[26] F. Golse, B. Perthame and R. Sentis, Un résultat de compacité pour les équations de transport et application au calcul de la valeur propre principale dun opérateur de transport, C. R. Acad. Sci. Paris 301 (1985), 341-344.

[27] F. Golse, P.L. Lions, B. Perthame and R. Sentis, Regularity of the moments of the solution of a transport equation, Journal of Func. Ana., 76, 110-125, 1988.

[28] G. W. Hammett, W. Dorland, and F. W. Perkins, Fluid models of phase mixing, Landau damping, and nonlinear gyrokinetics dynamics, Phys. Fluids, B, 4 (7), 1992, 2052-2061.

[29] P.D. Hislop, I.M. Sigal : Introduction to Spectral Theory With applications to Schrödinger Operators, Applied Mathematical Sciences 113. Springer, 1995.

[30] J.-H. Hwang and J. Velazquez, On the existence of exponentially decreasing solutions of the nonlinear Landau damping problem, Indiana Univ. Math. J. 58 (2009), no. 6, 2623-2660.

[31] I. H. Hutchinson, Electron holes in phase space: What they are and why they matter, Physics of Plasmas 24, 055601 (2017).

[32] P.E. Jabin, and A. Nouri, Analytic solutions to a strongly nonlinear Vlasov equation. C. R. Math. Acad. Sci. Paris 349, no. 9-10, 541-546, 2011.

[33] T. Kato, Wave operator and unitary equivalence, Pacific journal of mathematics, 15, 1, 1965.

[34] A.J. Klimas and J. Cooper, Vlasov-Maxwell and Vlasov-Poisson equations as models of a one-dimensional electron plasma, Phys. Fluids 26, 478-480 (1983).

[35] B. O. Koopman, Hamiltonian systems and transformations in Hilbert space, Proc. Nat. Acad. Sci. USA 17, 315 (1931).

[36] M.D. Kruskal and C.R. Oberman, On the stability of plasma in static equilibrium, Phys. Fluids, 1 (4), 275-280, 1958.

[37] L. Landau, J . Phys. USSR, 10, (1946) 25.

[38] P.D. Lax and R.S. Phillips, Scattering Theory, Academic Press, 1967. 
[39] M. Lemou, F. Mehats, P. Raphael, A new variational approach to the stability of gravitational systems, Comm. Math. Phys. 302 (2011).

[40] G. Manfredi, Long time behavior of nonlinear Landau damping, Phys. Rev. Lett. 79-15, 2815, 1997.

[41] Z. Lin and W. Strauss, Nonlinear stability and instability of relativistic VlasovMaxwell systems, Comm. Pure Appl. Math., 60 (2007), 6, 789-837.

[42] Z. Lin and W. Strauss, Linear stability and instability of relativistic VlasovMaxwell systems, Comm. Pure Appl. Math., 60 (2007), 5, 724-787.

[43] Z. Lin and C. Zeng, Small BGK Waves and Nonlinear Landau Damping, Commun. Math. Phys. 306, 291-331 (2011).

[44] T. Li, D. Wei and Z. Zhang, Pseudospectral and spectral bounds for the Oseen vortices operator, Preprint arxiv:1701.06269v1, 2017.

[45] P. J. Morrison, Hamiltonian Description of Vlasov Dynamics: Action-Angle Variables for the Continuous Spectrum, Transport theory and statistical physics, 29(3-5), 397-414, 2000.

[46] P. J. Morrison and D. Pfirsch, Dielectric energy versus plasma energy, and Hamiltonian action-angle variables for the Vlasov equation, Phys. Fluids B 4 (10), 1992.

[47] C. Mouhot, Stabilité orbitale pour le système de Vlasov-Poisson gravitationnel, Séminaire Bourbaki, 2012, number 1044.

[48] C. Mouhot and C. Villani, On Landau damping, Acta Math. 207 (2011), no. $1,29-201$.

[49] M. Reed and B. Simon, Methods of modern mathematical physics: III Scattering theory, Academic Press, 1979.

[50] G. Rein, Collisionless Kinetic Equations from Astrophysics-The VlasovPoisson System. Handbook of Differential Equations, Evolutionary Equations. Vol. 3., Elsevier, 2007.

[51] E.M. Stein, Singular integrals and differentiability of functions, Princeton Mathematical Series, No. 30, 1970.

[52] N.G. Van Kampen, On the theory of stationary waves in plasmas, Physica XXI, 949-963, 1955.

[53] J. v. Neumann, Zur Operatorenmethode in der klassischen Mechanik, (German) Ann. of Math. (2) 33, no. 3, 587-642, 1932.

[54] J. v. Neumann, Züsatze zur Arbeit zur Operatorenmethode in der klassischen Mechanik, Ann. of Math. 2, 789-791, 1932.

[55] D. Yafaev, Scattering Theory: Some Old and New Problems, Lecture Notes Math. 1735, Springer-Verlag, Berlin, 2000.

Bruno Després

Sorbonne Universités, UPMC Univ Paris 06, UMR 7598, Laboratoire Jacques-Louis Lions, F-75005, Paris, France, Institut Universitaire de France e-mail: despres@ljll.math.upmc.fr 